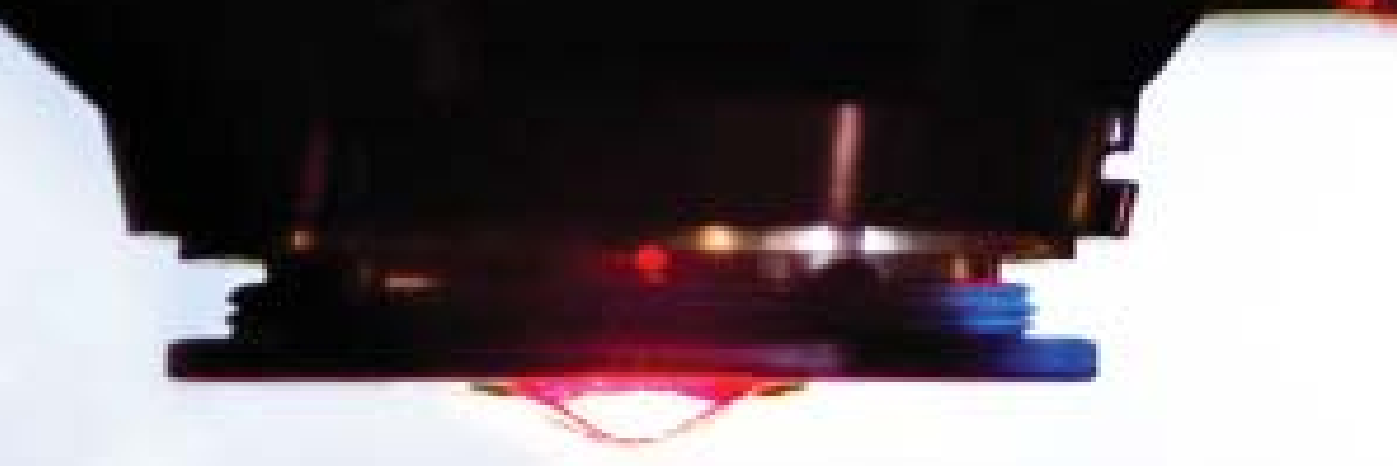

ELLIPSOMETRY BASED IMAGING TECHNIQUES FOR NANOSCALE CHARACTERIZATION OF HETEROGENEOUS POLYMER FILMS
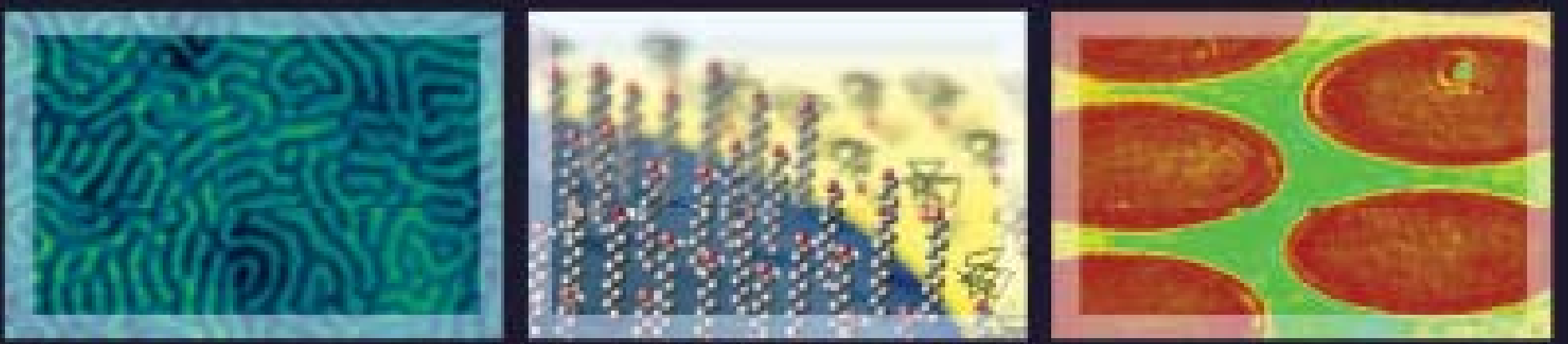

Aysegul Cumurcu 
Ellipsometry based imaging techniques for nanoscale characterization of heterogeneous polymer films 
The work described in this thesis has been performed at the Materials Science and Technology of Polymers (MTP) group, which is part of the Faculty of Science and Technology at the University of Twente, Enschede, The Netherlands.

Members of the committee:

Chairman Prof. dr. Hans Hilgenkamp

Promotor Prof. dr. G. Julius Vancso

Assistant-promotor Dr. Peter M. Schön

Members
Prof. dr. Fausto Sanz

Prof. dr. Szczepan Zapotoczny

Prof. dr. Kitty Nijmeijer

Prof. dr. Harold J. W. Zandvliet

Prof. dr. Pepijn W. H. Pinkse
University of Twente, University of Twente, University of Twente, University of Barcelona, Jagiellonian University, University of Twente, University of Twente, University of Twente.

ISBN: 978-90-365-3740-7

DOI: $10.3990 . / 1.9789036537407$

This research forms part of the research programme of the Dutch Polymer Institute (DPI), Corporate Research Technology, DPI project \#695. This research was also financially supported by the $\mathrm{MESA}^{+}$Institute for Nanotechnology of the University of Twente.

\section{DPI}

Copyright (C)Aysegul Cumurcu, Enschede, The Netherlands 2014.

No part of this work may be reproduced by print, photocopy, or any other means without permission in writing from the publisher.

Cover design by Maurice Vlot.

Printed by Ipskamp Drukkers in Enschede, The Netherlands. 


\title{
ELLIPSOMETRY BASED IMAGING TECHNIQUES FOR NANOSCALE CHARACTERIZATION OF HETEROGENEOUS POLYMER FILMS
}

\author{
PROEFSCHRIFT
}

ter verkrijging van

de graad van doctor aan de Universiteit Twente, op gezag van de rector magnificus, prof. dr. H. Brinksma, volgens besluit van het College voor Promoties, in het openbaar te verdedigen op vrijdag 26 september 2014 om 16.45 uur

$$
\text { door }
$$

\section{Aysegul Cumurcu}

geboren op 12 oktober 1981 te Istanbul, Turkije 
Dit proefschrift is goedgekeurd door:

Promotor: Prof. dr. G.J. Vancso

Assistent promotor: Dr. P.M. Schön 


\section{Contents}

1 Hybrid methods for nanoscale characterization of polymer films 1

1.1 Introduction . . . . . . . . . . . . . . . . . . 2

1.2 Concept of this thesis $\ldots \ldots \ldots \ldots \ldots \ldots$

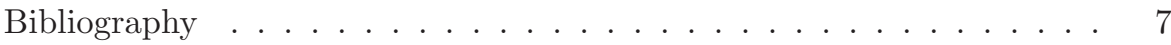

2 Far and near field ellipsometric microscopy techniques 9

2.1 Optical microscopy . . . . . . . . . . . . . . . . . . . . . . . 10

2.1.1 Diffraction limit . . . . . . . . . . . . . . . . . 10

2.1.2 Far-field microscopy with sub diffraction resolution . . . . . 11

2.1.3 Applications of far-field microscopy with sub diffraction resolution . . . . . . . . . . . . . . . . 13

2.1.4 Near-field microscopy _. . . . . . . . . . . . . . . . . 15

2.2 Imaging ellipsometry . . . . . . . . . . . . . . . . . . . . . . 17

2.2 .1 Introduction . . . . . . . . . . . . . . . 17

2.2 .2 Instrumentation . . . . . . . . . . . . . . . . . . . 19

2.2.3 Selected applications of imaging ellipsometry . . . . . . . 21

2.3 Scanning near-field optical microscopy . . . . . . . . . . . . 26

2.3.1 Aperture-based near-field optical microscopy . . . . . . . . 26

2.3.2 Apertureless near-field optical microscopy . . . . . . . . . 28

2.4 Conclusions . . . . . . . . . . . . . . . . . . . . . . . . 38

Bibliography . . . . . . . . . . . . . . . . . 39

3 Imaging ellipsometry of redox-responsive polymer films 45

3.1 Introduction . . . . . . . . . . . . . . . . 47

3.2 Results and discussion . . . . . . . . . . . . . . . . . . . 49

3.2.1 Micro-patterned ES-PFS/MCU film preparation . . . . . . 49

3.2.2 Electrochemistry of the ES-PFS/MCU sample . . . . . . 51

3.2 .3 Thickness measurements by EC-IE . . . . . . . . . . . 53

3.2.4 In situ ellipsometric contrast images . . . . . . . . . . . . 54

3.2 .5 AFM topography imaging . . . . . . . . . . . . 57

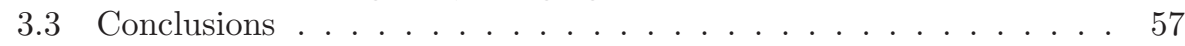

3.4 Experimental section . . . . . . . . . . . . . . . . . 58

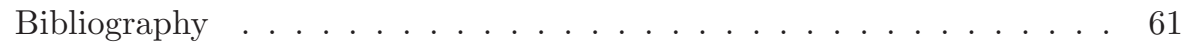


4 Scanning near-field ellipsometric microscopy setup 65

4.1 Introduction . . . . . . . . . . . . . . . . . 67

4.2 Principle of the SNEM setup _ . . . . . . . . . . . . . . . 69

4.3 Point dipole model . . . . . . . . . . . . . . . . . . . . 72

4.4 Contribution of the far-field signal . . . . . . . . . . . 73

4.5 Conclusions . . . . . . . . . . . . . . . . . 75

4.6 Experimental section . . . . . . . . . . . . . . . . . 75

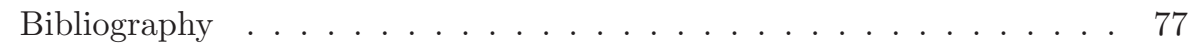

5 Multimodal imaging of PS- $b$-P2VP block copolymer films 81

5.1 Introduction . . . . . . . . . . . . . . . . . 83

5.2 Results and discussion . . . . . . . . . . . . . . . 85

5.2.1 Comparing the gold coated tip with a bare silicon tip . . 85

5.2 .2 Staining of the polymer film . . . . . . . . . . . . 88

5.2 .3 NAP images and model calculations . . . . . . . . . . . 89

5.3 Conclusions . . . . . . . . . . . . . . . . . . . . . . . . . 92

5.4 Experimental section . . . . . . . . . . . . . . . . . 93

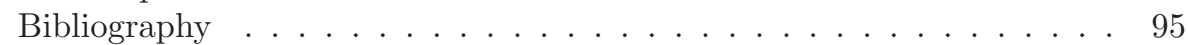

6 SAM modification of gold coated AFM probes 97

6.1 Introduction . . . . . . . . . . . . . . . . . . . . . . 99

6.2 Results and discussion . . . . . . . . . . . . . . . . . . 100

6.2.1 Degradation of a gold coated AFM probe tip . . . . . . . 100

6.2.2 Protection of the gold coated AFM probe tips with SAM . 103

6.2.3 SAM modification of gold coated tips with various thiols . . 105

6.3 Conclusions . . . . . . . . . . . . . . . . . . . . 107

6.4 Experimental section . . . . . . . . . . . . . . . . . . . 107

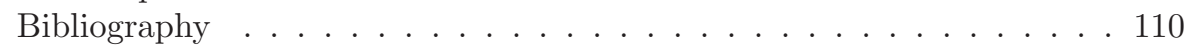

7 Optical signal blocking and recovery in SNEM 113

7.1 Introduction . . . . . . . . . . . . . . . . . . . 115

7.2 Results and discussion . . . . . . . . . . . . . . . 116

7.2.1 SNEM imaging using a SAM of 1-dodecanethiol function-

alized tip . . . . . . . . . . . . . . . . 117

7.2.2 Gold deformation at the tip apex . . . . . . . . . . . . 119

7.2.3 SNEM intensity-distance curves . . . . . . . . . . . . . . 121

7.3 Conclusions . . . . . . . . . . . . . . . . . . . . . 122

7.4 Experimental section . . . . . . . . . . . . . . . . . . . . 122

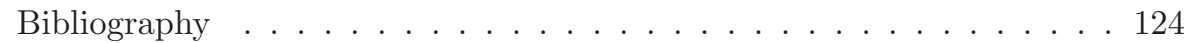


8 Outlook for EC-IE and SNEM $\quad 127$

8.1 Introduction . . . . . . . . . . . . . . . . . . . 128

8.2 Electrochemical imaging ellipsometry . . . . . . . . . . . . . . 129

8.3 Scanning near-field ellipsometric microscopy . . . . . . . . . . . . . 129

8.4 Conclusions . . . . . . . . . . . . . . . . . . . . 134

8.5 Experimental section . . . . . . . . . . . . . . . . 134

Bibliography ....................... 136

$\begin{array}{ll}\text { Summary } & 137\end{array}$

$\begin{array}{lr}\text { Samenvatting } & 141\end{array}$

$\begin{array}{lr}\text { Acknowledgements } & 145\end{array}$

$\begin{array}{lr}\text { Curriculum Vitae } & 147\end{array}$ 


\section{Chapter 1}

\section{Hybrid methods for nanoscale characterization of polymer films}

In this thesis, two ellipsometry based hybrid microscopy techniques for nanoscale characterization of thin polymer films are introduced. Firstly, an ellipsometer is combined with electrochemical methods for the visualization of morphology changes of a redox-active polymer film. Secondly, an ellipsometer is combined with an AFM for nanoscale optical imaging of heterogeneous thin polymer films. Both hybrid techniques improve the capabilities of each instrument enabling advanced inspection of dynamic morphology changes and optical contrast variations in thin polymer films at the nanoscale. In this chapter, an overview of the thesis is provided. 


\subsection{Introduction}

Swift developments in nanotechnology necessitate the use of advanced characterization techniques with nanoscale resolution. Electron microscopy (EM), scanning probe microscopy (SPM, such as atomic force microscopy, AFM, and scanning tunneling microscopy, STM) and modern far-field fluorescence based microscopies (stochastic optical reconstruction microscopy, STORM, stimulated emission depletion, STED, and photoactivated localization microscopy, PALM) are among the modern microscopy techniques that provide nanoscale resolution [1-7].

In recent years, two or three complementary techniques have been combined into hybrid devices as more versatile orthogonal techniques to extract a variety of local information such as morphological, (electro)chemical, mechanical and kinetic data simultaneously [8]. Examples of such devices include AFM combined with Raman spectroscopy (tip-enhanced Raman spectroscopy, TERS), AFM combined with ellipsometry (scanning near-field ellipsometric microscopy, SNEM), AFM combined with electrochemistry (electrochemical AFM, EC-AFM), electrochemistry combined with ellipsometry/imaging ellipsometry (EC-IE) [9-12].

Combining AFM with Raman spectroscopy provides the capability of obtaining morphological and chemical information simultaneously [13, 14]. TERS combines surface enhanced Raman spectroscopy (SERS) with AFM analysis to obtain nanometer scale spatial chemical resolution. Using the advantage of SERS, providing orders of magnitude increase in Raman signal intensity, with the localization capabilities of the AFM tip, a Raman signal is enhanced and localized to nanometer scale.

AFM combined with ellipsometry provides topography and optical property information at the nanoscale for characterization of heterogeneous films $[10,15,16]$. SNEM uses the ellipsometer's ability to measure the polarization state of the light with high precision. The metallic AFM probe tip is used to localize, enhance and scatter the electromagnetic field. The change in the polarization state of the reflected light due to the interactions between the tip-sample coupled system is transferred to contrast in the recorded image simultaneously with the AFM data.

Combining AFM with electrochemical methods adds local electrochemical capabilities to AFM and allows one to obtain redox activity data with topographical information $[11,17,18]$. In EC-AFM, topography imaging is recorded under electrochemical control by applying a potential to the sample. AFM images can be recorded at constant potential or during potential cycling. Thus, electrochemically induced surface changes are captured in situ at the electrode/electrolyte interface. Consequently, combining ellipsometry or IE with electrochemical methods allows one to measure dynamic thickness and morphology changes of a film due to an electrochemical stimulus, for instance protein adsorption on charged surfaces and film growth by cyclic voltammetry (CV) based deposition techniques $[12,19]$. 
In this thesis, two hybrid methods are introduced, namely IE combined with electrochemical techniques, EC-IE, and AFM combined with ellipsometry, SNEM. Primarily, a case study is presented utilizing the EC-IE setup (Fig. 1.1). In particular, IE was combined with electrochemical methods to study the dynamic response of a redox-active polymer. In this combined method, the ellipsometry was used in imaging configuration. This hybrid device allows one to determine the thickness and morphology variations of polymer films with sub-nanometer resolution, since it uses the polarization state of the light which is sensitive to the changes of thickness and refractive index in thin layers.

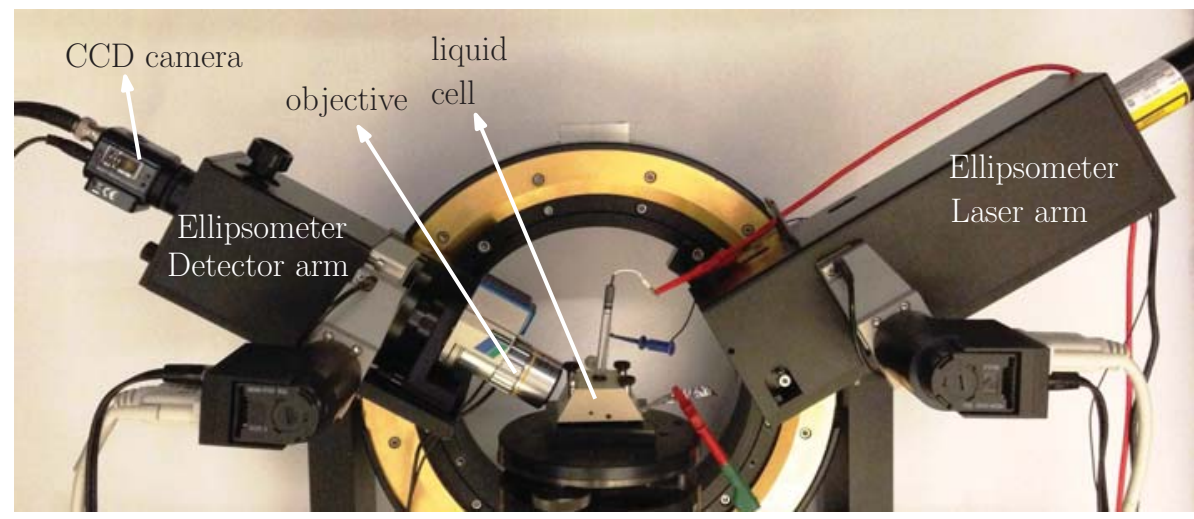

Figure 1.1: A photograph of the EC-IE setup. A CCD camera and an objective were implemented to an ellipsometer for imaging mode. The sample was placed in a liquid cell. A gold substrate served as a working electrode. $\mathrm{A} \mathrm{Ag} / \mathrm{AgCl}$ and a $\mathrm{Pt}$ wire were used as a reference and a counter electrode, respectively.

IE consists of an objective based light microscope that contains a laser source in the visible region, therefore, it is diffraction limited and cannot provide nanoscale resolution in the lateral direction. In the second combined method, however, nanoscale resolution is achieved owing to the light confinement capability of the metal coated AFM probe tip. In this near-field optical microscope, the same light source of the IE equipment is used. However, in this combination of ellipsometry with AFM, the lateral resolution is no longer limited by the diffraction limit of the light, but limited by the diameter of the nanoscale metallic probe. Accordingly, the thesis focuses first on the introduction to our instrumental SNEM configuration (Fig. 1.2). Then, more information and examples in form of case studies to unravel the contrast mechanism of the hybrid technique are provided. By combining different techniques, the capabilities of these individually well known methods were extended. In the EC-IE method, in situ real time imaging of the response of a polymer film to the electrochemical stimuli was obtained. In the SNEM method, topography and optical contrast information of thin heterogeneous polymeric films was collected simultaneously with nanoscale resolution. 


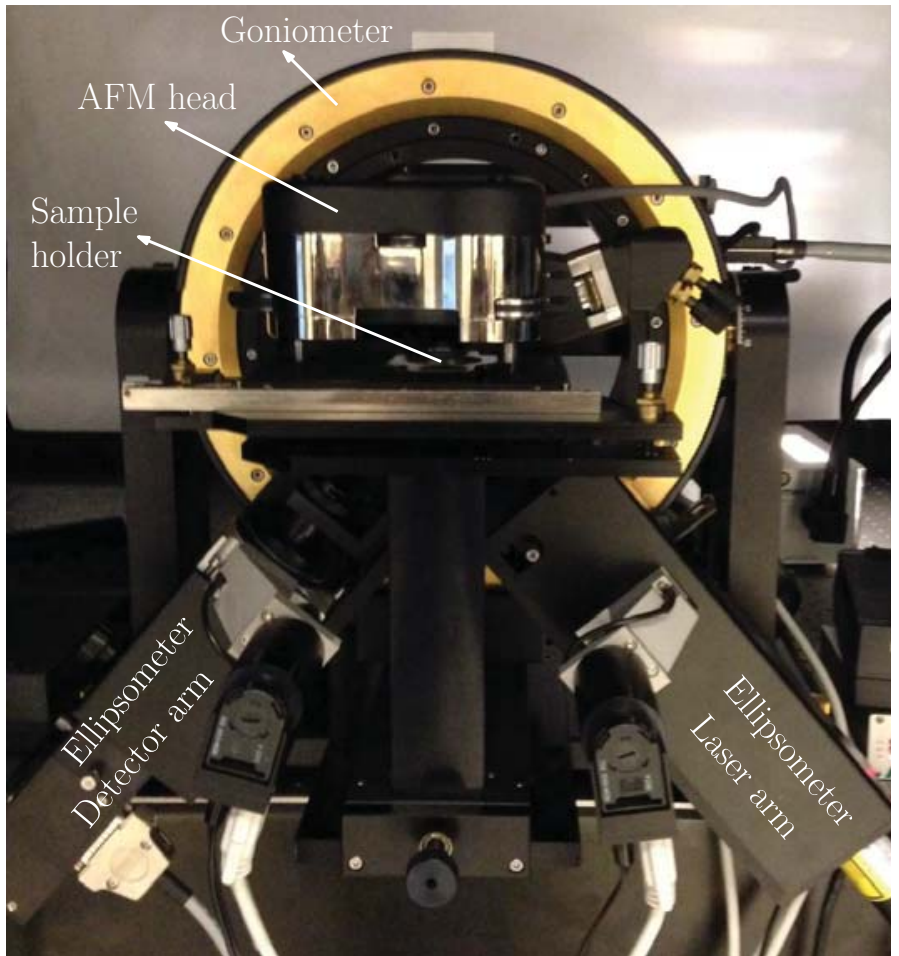

Figure 1.2: A photograph of the SNEM setup. An AFM is coupled to an ellipsometer. SNEM optical images were collected in air.

\subsection{Concept of this thesis}

A summary of recent developments, the fundamentals of IE and various scanning near-field optical microscopy (SNOM) techniques are provided in Chapter 2. The chapter starts with the explanation of diffraction limitation of conventional lens based optical microscopy and examples in recent developments, such as in fluorescence microscopy, which use various sophisticated physical concepts to defeat the diffraction limit of light. IE, which is a diffraction limited optical microscopy, is introduced including various examples presented as case studies. For instance, IE was used for polymer characterization for measurements in thickness distribution and thermoresponsive aggregation [20,21]. Subsequently, the chapter focuses on near-field optical microscopy techniques. The apertureless and apertured SNOM techniques are described placing the focus mainly to TERS and SNEM.

In Chapter 3, an EC-IE, which combines IE with electrochemical methods, is utilized to monitor in situ morphology changes of an oligoethylene sulfide endfunctionalized poly(ferrocenyldimethylsilane) (ES-PFS). Combining IE with electrochemical methods provides non-distractive real time tracking of the thickness and morphology changes triggered by variations in the redox state of the poly- 
mer film with sub-nanometer accuracy. In this study, a non-redox responsive 11-mercapto-1-undecanol (MCU) layer was used as a reference layer. A micropatterned sample of MCU and ES-PFS layers was produced by micro-contact printing $(\mu \mathrm{CP})$. The ellipsometric contrast images were recorded in real time by IE under electrochemical control to monitor intensity changes in the ES-PFS layer when switched from the reduced to the oxidized state.

IE provides sub-nanometer vertical sensitivity, but its lateral resolution is limited by the wavelength of the light source and the numerical aperture of the objective used. In contrast, SNEM, which is a tip enhanced technique comprised of an AFM and an ellipsometer, the lateral resolution is not limited by the wavelength of the light, but limited by the diameter of the probe tip used. The experimental arrangement of the SNEM configuration is provided in Chapter 4. This chapter also covers the explanation of a model termed point dipole model which calculates the scattering response of a tip-sample coupled system in apertureless SNOM. This model provides the basis for contrast formation and is used in this thesis to compare the experimental results with the model calculations. Additionally, the far-field background scattering which depends interferometrically on the vertical tip position is explained.

A deeper insight into the nature of the SNEM contrast mechanism is provided in Chapter 5. A microphase separated poly(styrene- $b$-2-vinyl pyridine) (PS$b$-P2VP) diblock copolymer film was studied with a gold coated and a silicon AFM probe tip to investigate the effect of the metal coating on the optical signal enhancement. Furthermore, the amplitude of intensity variations in SNEM images arising from the periodic domain pattern formed by the diblock copolymer as function of increasing tip-sample separation was studied by comparing the SNEM optical images captured at various tip-sample distances. Additionally, the point dipole model calculations were compared with the experimental results.

In Chapter 6, the degradation of a gold coated AFM probe, which is a common problem in apertureless SNOM using metallic tips, is investigated. Gold coated AFM probe tips were shown to provide unstable tip activity over time when stored in ambient air. A protective coating of a self-assembled monolayer (SAM) of ethanethiol (EtSH) slowed down the degradation of the gold coated AFM probe tips when stored under the same conditions. The SAM of EtSH protected the AFM probe tips from accumulation of surface contaminants during storage in ambient air.

SNEM images of a microphase separated polystyrene-block-poly (methylmethacrylate) (PS-b-PMMA) diblock copolymer film, were collected with a bare gold coated and SAM protective gold coated AFM probes both, immediately after coating and following five days of storage in ambient air. The intensity variations of the SNEM images were compared to determine the degradation of the tips. Scanning electron microscopy (SEM) images of the fresh and degraded AFM probe tips were compared to provide more information on the morphology change of the tips over time. Lastly, gold coated AFM probe tips were modified with alkanethiols of 
various lengths and the intensity variations of the SNEM images were compared. The point dipole model was used to explain the decrease in the optical image by increasing the chain length of the thiol molecules.

The blocking and recovering of the optical signal is investigated in Chapter 7. A long chain thiol SAM of 1-dodecanethiol (DoSH) was used to completely block the optical contrast including the contribution of the unwanted reflections from the tip shaft. The SAM at the apex of the tip was then locally removed by deforming the molecules under load. Intensity-distance curves recorded with a bare gold coated and a DoSH modified AFM probe tip were collected to confirm the decrease of the background scattering via the SAM. Lastly, an outlook with discussions of the possibilities for future research of both hybrid methods is provided in Chapter 8 . 


\section{Bibliography}

[1] Schatten, H. Micron 2011, 42, 175-185.

[2] Firtel, M.; Beveridge, T. Micron 1995, 26, 347-362.

[3] Busko, D.; Baluschev, S.; Crespy, D.; Turshatov, A.; Landfester, K. Micron 2012, 43, 583-588

[4] Rust, M. J.; Bates, M.; Zhuang, X. W. Nat. Methods 2006, 3, 793-795.

[5] Brown, T. A.; Fetter, R. D.; Tkachuk, A. N.; Clayton, D. A. Methods 2010, $51,458-463$.

[6] Sotthewes, K.; Geskin, V.; Heimbuch, R.; Kumar, A.; Zandvliet, H. J. W. APL Mat. 2014, 2, 010701 1-11.

[7] Zapotoczny, S.; Biedron, R.; Marcinkiewicz, J.; Nowakowska, M. J. Mol. Recogn. 2012, 25, 82-88.

[8] Flores, S. M.; Toca-Herrera, J. L. Nanoscale 2009, 1, 40-49.

[9] Pettinger, B.; Schambach, P.; Villagomez, C. J.; Scott, N. Annu. Rev. Phys. Chem. 2012, 63, 379-399.

[10] Karageorgiev, P.; Orendi, H.; Stiller, B.; Brehmer, L. Appl. Phys. Lett. 2001, 79, 1730-1732.

[11] Zou, S.; Hempenius, M. A.; Schönherr, H.; Vancso, G. J. Macromol. Rapid. Comm. 2006, 27, 103-108.

[12] Svoboda, V.; Cooney, M. J.; Rippolz, C.; Liaw, B. Y. J. Electrochem. Soc. 2007, 154, D113-D116.

[13] Schmid, T.; Opilik, L.; Blum, C.; Zenobi, R. Angew. Chem. Int. Edit. 2013, 52, 5940-5954.

[14] Tian, Z. Q.; Ren, B.; Li, J. F.; Yang, Z. L. Chem. Commun. 2007, 3514-3534.

[15] Tranchida, D.; Diaz, J.; Schön, P.; Schönherr, H.; Vancso, G. J. Nanoscale 2011, 3, 233-239.

[16] Cumurcu, A.; Duvigneau, J.; Lindsay, I. D.; Schön, P. M.; Vancso, G. J. Eur. Polym. J. 2013, 49, 1935-1942.

[17] Peter, M.; Hempenius, M. A.; Kooij, E. S.; Jenkins, T. A.; Roser, S. J.; Knoll, W.; Vancso, G. J. Langmuir 2004, 20, 891-897.

[18] Kelley, S. O.; Barton, J. K.; Jackson, N. M.; McPherson, L. D.; Potter, A. B.; Spain, E. M.; Allen, M. J.; Hill, M. G. Langmuir 1998, 14, 6781-6784.

[19] Yu, Y.; Jin, G. J. Colloid Interf. Sci. 2005, 283, 477-481. 
[20] Abrantes, L. M.; Correia, J. P.; Savic, M.; Jin, G. Electrochim. Acta. 2001, $46,3181-3187$.

[21] Schmaljohann, D.; Nitschke, M.; Schulze, R.; Eing, A.; Werner, C.; Eichhorn, Y. J. Langmuir 2005, 21, 2317-2322. 


\section{Chapter 2}

\section{Far and near field ellipsometric microscopy techniques}

This chapter provides an overview of recent developments concerning imaging ellipsometry (IE) and various scanning near-field optical microscopy (SNOM) techniques. In the beginning, diffraction limit of conventional far field (lens based) optical microscopy is explained. Next, recent developments in far field based fluorescence microscopy utilizing various sophisticated physical concepts to achieve optical resolution below the diffraction limit are discussed. Subsequently, IE is introduced belonging as well to the family of diffraction limited optical microscopy. Various case studies are discussed utilizing IE for polymer characterization. Lastly, the main part of this chapter is dedicated to near-field optical microscopy. A brief overview of apertureless and apertured SNOM is provided. Particular attention is paid to tip enhancement techniques, focusing on tip-enhanced Raman scattering (TERS) and scanning near-field ellipsometric microscopy (SNEM). 


\subsection{Optical microscopy}

Structure and morphology of materials at length scales not visible for the naked eye are usually visualized by various microscopy techniques $[1,2]$. The invention of the first useful optical microscope by van Leeuwenhoek in the $17^{\text {th }}$ century revolutionized biology and natural sciences at the micrometer length scale (Fig. 2.1) [3]. Various forms of optical microscopies have since then become enabling methods to investigate synthetic and biological matter. The quest for visualizing smaller and smaller details of materials resulted in the development of electron microscopies, field-ion microscopies and recently scanning probe microscopies [4-6].

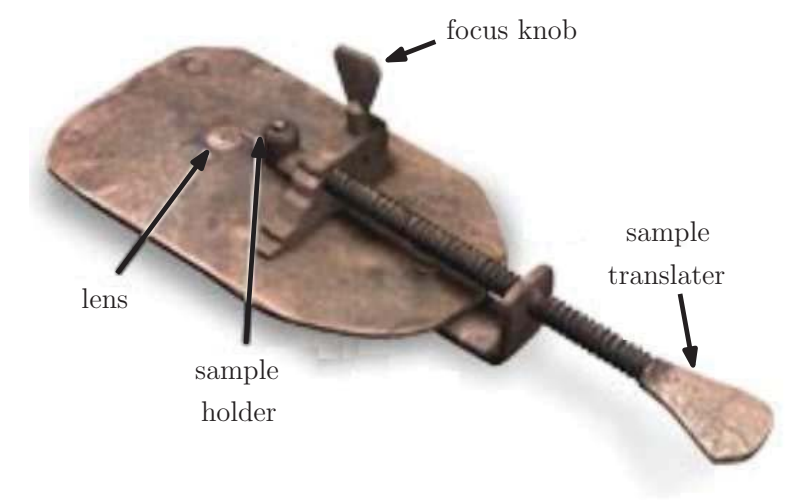

Figure 2.1: Illustration of the microscope of van Leeuwenhoek. The design consists of a simple magnifying glass. The specimen was mounted on a sharp point in front of the lens. The position of the sample and the focus could be adjusted by turning the two screws. From reference [3].

\subsubsection{Diffraction limit}

Swift progress in nanotechnology makes it essential to study optical phenomena at the nanometer scale [7]. Since light cannot be focused to sizes smaller than roughly one half of the wavelength due to the diffraction limit, conventionally it is not possible to characterize nanoscale features with optical methods [8]. The diffraction, a phenomenon that occurs when a wave encounters an obstacle, is described as the apparent bending of waves around small objects and divergence of the waves when a small opening is passed. Since most specimens observed in the microscope are composed of highly overlapping features, the diffraction property of light restricts the ability of the optical instruments to distinguish these features. Ernst Abbe described the required distance between two objects that can be resolved as 


$$
d=\frac{\lambda}{2 n \sin \alpha},
$$

where $\lambda$ is the wavelength of the emitted light, $n$ is the refractive index of the medium and $\alpha$ is the angular aperture of the microscope, [9]. If the microscope could detect light approaching from all directions, the term $\sin \alpha$ would drop and in the case of $n=1$, the resolution limit would become equal to the half of the wavelength of the emitted light. However, in practice, conventional microscopes do not reach this theoretical resolution limit, since they cannot detect light from all directions and contain imperfections in the optical lenses [10, 11].

\subsubsection{Far-field microscopy with sub diffraction resolution}

The far-field light used in conventional microscopy propagates through space in an unconfined manner. The scattering of electromagnetic radiation involves diffraction and reflection upon interacting with the specimen being collected in the far field to form an image. With fluorescence based imaging methods it is possible to reconstruct an image of objects that are significantly smaller than the ones observed by conventional optical microscopy by exploiting the photophysics of extrinsic fluorophores [12]. However, their resolution still strongly depend on the shape of the optical focus, which is determined by conventional lens systems and therefore subjected to the diffraction limit.

In recent years, a variety of fluorescence microscopies have been developed that can overcome the diffraction limit by utilizing various sophisticated physical concepts that will be elaborated in the following. Importantly, nanoscale optical resolution has been established and the era of far-field nanoscopy has since then experienced a tremendous and ongoing development $[13,14]$. Here a short overview is provided.

The most important high spatial resolution fluorescence microscopy techniques include structured illumination microscopy (SIM), stimulated emission depletion microscopy (STED), photoactivated localization microscopy (PALM) and stochastic optical reconstruction microscopy (STORM) [15-18]. SIM is based on the Moiré fringes generated by the interactions between the illuminated light pattern and the fluorescent probes in the sample. The light pattern is translated and rotated to collect a series of images which are then reconstructed to obtain a high resolution final image $[15,19]$. STED is a fluorescence microscopy technique that provides fluorescence focal spots of sub-diffraction dimensions by transiently depleting the fluorescence throughout the focal region except a tiny area at the center of the excitation spot by using a second doughnut-shaped light beam $[16,20]$. PALM is based on the stochastic photoactivatation of fluorophores and the images are collected in cycles of activation and bleaching of small groups of fluorophores [17, 21]. In STORM, activation and bleaching of 
the photoswitchable fluorophores is performed using light with different wavelengths [18, 22]. Schematic representation of the confocal fluorescence microscopy, STED and PALM/STORM are shown in Fig. 2.2, [14].

(a) Confocal
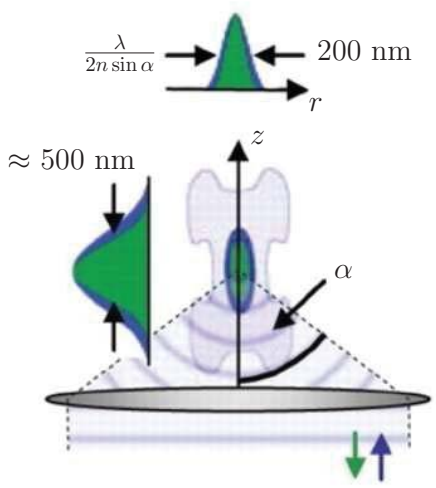

(b) STED

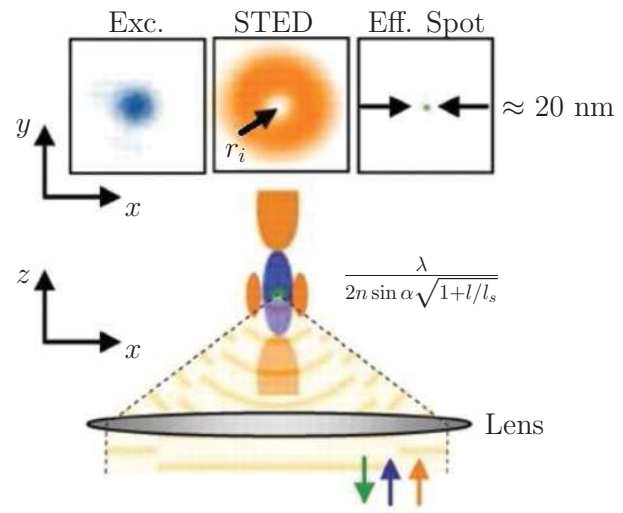

(c) PALM/STORM

Switch single molecules
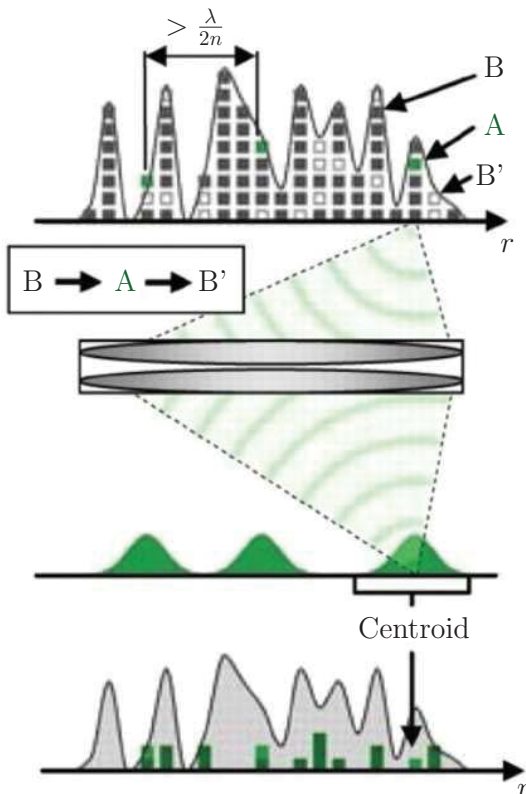

Stochastic read-out

Figure 2.2: Scheme of (a) confocal fluorescence microscopy, (b) STED and (c) PALM/STORM. The resolution of the confocal optical microscopy is limited by the diffraction to $>200 \mathrm{~nm}$ in the focal plane (x, y) and to $>450 \mathrm{~nm}$ along the optical ( $\mathrm{z}$ ) axis. In STED microscopy, a regularly focused excitation beam (blue) and a doughnut-shaped STED beam (orange) are superimposed resulting in a sub-diffraction detection area (green). In PALM, the individual fluorophores with distances of $\lambda / 2 n$ switch stochastically to the bright state A. Reprinted with permission from reference [14]. Copyright 2007 American Association for the Advancement of Science. 


\subsubsection{Applications of far-field microscopy with sub diffrac- tion resolution}

The ability of STED far-field fluorescence nanoscopy to study dynamics of biomolecules in living cells was shown by Eggeling and Ringemann et al. [23]. Diffusion of sphingomyelin (SM) lipids and phosphoethanolamine (PE) in the plasma membrane of living cells were studied by targeting arbitrary points on the plasma membrane and recording the fluorescence bursts of molecules crossing the focal spot.

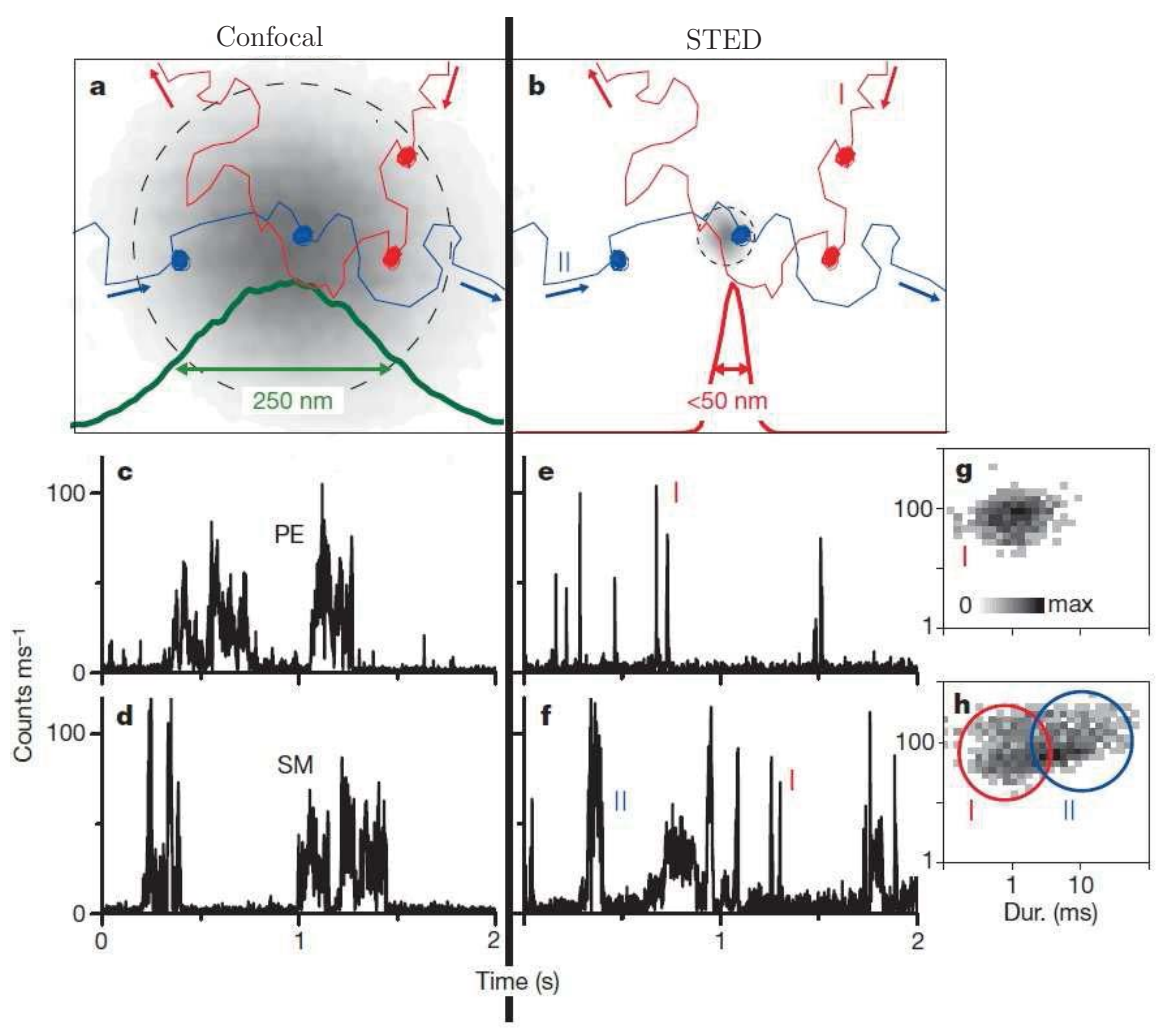

Figure 2.3: STED scheme of the detection area and the time traces data. In (a) and (b), the schemes for the detection area of a confocal and a STED microscope are shown, respectively. The fluorescence bursts from single-diffusing PE and SM lipids detected with a confocal microscope are depicted in (c) and (d), respectively. In (e) and (f), the same measurements detected with the STED are shown for PE and SM lipids, respectively. A frequency histogram of the fluorescent counts per millisecond along with the burst duration for about 500 bursts recorded for PE and SM with the STED is displayed in (g) and (h). Reprinted with permission from reference [23]. Copyright 2009 Macmillan Publishers Limited. 
Single-molecule fluoresecence time traces of PE and SM were measured by the regular confocal and the sub-diffraction detection area downsized by STED to approximately $40 \mathrm{~nm}$ (Fig. 2.3). The STED measurements showed a range of burst durations for SM which were noticeably different from the measurements of PE, whereas the confocal measurements did not reveal such difference. The STED measurements revealed that the diffusion of SM is strongly heterogeneous, while no observable sign of heterogeneous diffusion was found for PE in the plasma membrane.

The axial and lateral positions of individual fluorophores were determined by 3D STORM with an image resolution of 20 to $30 \mathrm{~nm}$ in the lateral dimensions and 50 to $60 \mathrm{~nm}$ in the axial dimensions [24]. The 3D morphology of clathrin-coated pits (CCPs) in green monkey kidney epithelial (BS-C-1) cells were resolved by using the astigmatism imaging method in 3D STORM imaging (Fig. 2.4).
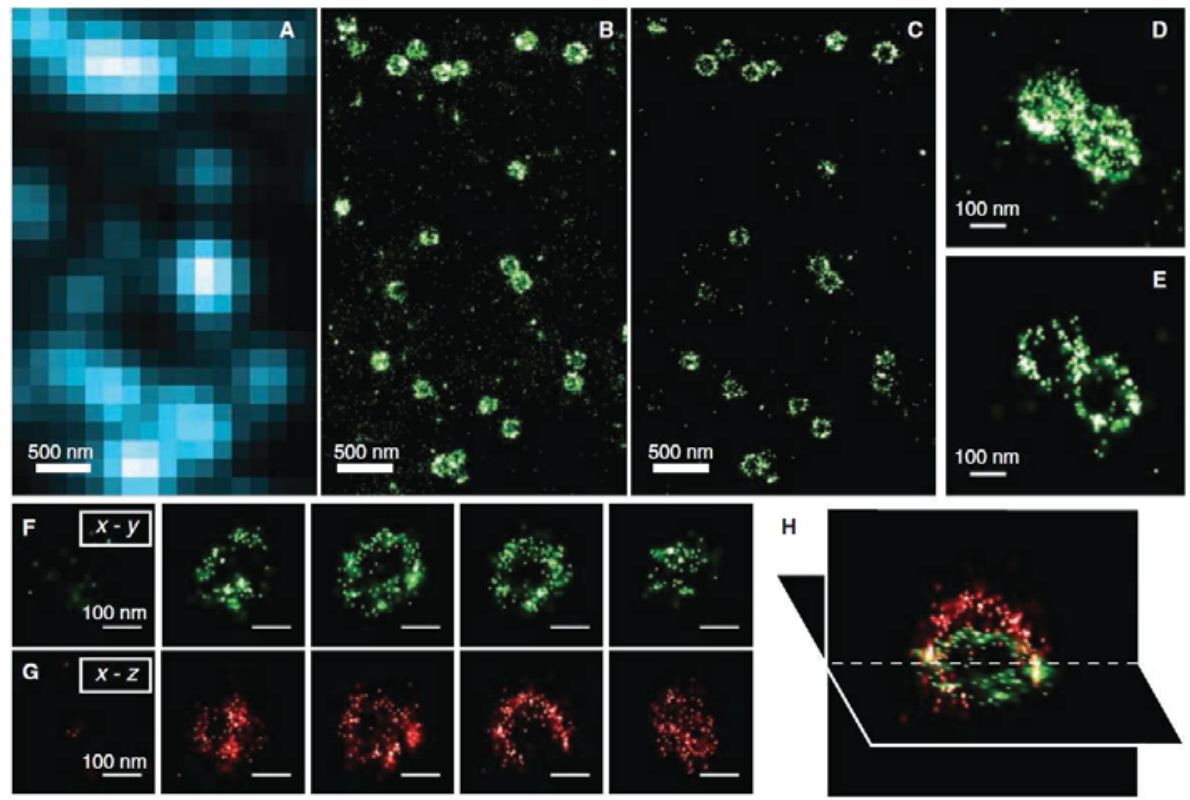

Figure 2.4: Conventional fluorescence microscopy and STORM images of CCPs in a cell. In (A), the direct immunofluorescence image of the CCPs is shown. In (B), the 2D STORM image of the same area with all locations at different $z$ positions is shown. In (C), the $x$-y cross-section of the same area at $z=50 \mathrm{~nm}$ is displayed. In (D), the 2D STORM image of a zoomed area is shown, while in (E) the $x-y$ cross-section of the 3D image at $z=100 \mathrm{~nm}$ is displayed for the same area. In (F), series of images of $x-y$ cross-sections are shown for every $50 \mathrm{~nm}$ in $z$ and the series of images of the $x$ - $z$ cross-sections for every $50 \mathrm{~nm}$ in $y$ are shown in $(\mathrm{G})$. In $(\mathrm{H})$, the $3 \mathrm{D}$ perspective image produced from the $x-y$ and $x-z$ cross-sections is displayed. Reprinted with permission from reference [24]. Copyright 2008 American Association for the Advancement of Science. 
The direct immunofluorescence image of CCPs in a region of a BS-C-1 cell captured with conventional microscopy did not show the detail structure of the CCPs since the resolution of the microscope is diffraction limited (Fig. 2.4(A)). However, in the 2D STORM image of the same area the round shape of the CCPs were revealed clearly (Fig. 2.4(B)). The size distribution of the CCPs were determined as $180 \pm 40 \mathrm{~nm}$ from the $2 \mathrm{D}$ projection image. The $3 \mathrm{D}$ structure of the CCPs are shown in Fig. $2.4(\mathrm{C})$ and $(\mathrm{E})$ to $(\mathrm{H})$. The $x-y$ and $x-z$ cross-sections revealed the half-spherical cage-like morphology of the CCPs (Fig. 2.4(F) to (H)). Consequently, nanoscopic features of cellular structures with molecular specificity were resolved by 3D STORM.

Shroff et al. investigated the nanoscale dynamics within individual adhesion complexes (ACs) in living cells with PALM images at spatial resolutions down to approximately $60 \mathrm{~nm}$ (Fig. 2.5) [25]. The formation of the ACs were visualized and the fractional gain and loss of individual paxillin molecules as each AC evolved were measured. The NIH 3T3 cell in Fig. 2.5(a) and (b) revealed inward AC motion toward the cell center. However, there were no new AC formation at the cell periphery. The (Chinese Hamster Ovary) CHO cell displayed in Fig. 2.5(c) and (d) showed inward motion and formation of peripheral ACs. For the NIH 3T3 cell, the motion of ACs near the periphery of the lamellum toward the interior and the subsequent dissolution as the cell retracted further was detected (Fig. 2.5(e) and (f)).

\subsubsection{Near-field microscopy}

The near-field light, a non-propagating field near the surface of an object, decays exponentially within a distance less than the wavelength of the light. The interactions between a sharp probe (mainly AFM or scanning probe microscope (SPM) probes) which is positioned at a very short distance from the sample surface, and a sample in the near-field region enables non-diffraction limited imaging and spectroscopy [26].

Scanning near-field optical microscopy (SNOM) is based on the enhanced photon flux between an apertured or apertureless probe and a sample [27, 28]. Therefore, the resolution depends on the diameter of the aperture or the tip of the probe rather than the wavelength of the light source (Fig. 2.6) [8]. The concept of using a sub-wavelength aperture probe to image a surface was first introduced by Synge in 1928 [29]. However, the lack of nanofabrication techniques at the time delayed the transformation of this idea into an experimental set-up until 1984 [30, 31]. Since then SNOM has become a pivotal high resolution characterization tool for heterogeneous systems in various fields. For instance, an organic semiconductor film (diindenoperylene, DIP) on Si was studied by a tip-enhanced near-field optical microscopy with a parabolic mirror microscope [32]. Optical details and confined spectroscopic information at single molecular layers were achieved by recording at $6 \times 10^{5}$ times enhanced photoluminescence (PL). The topography and the cor- 
(a)
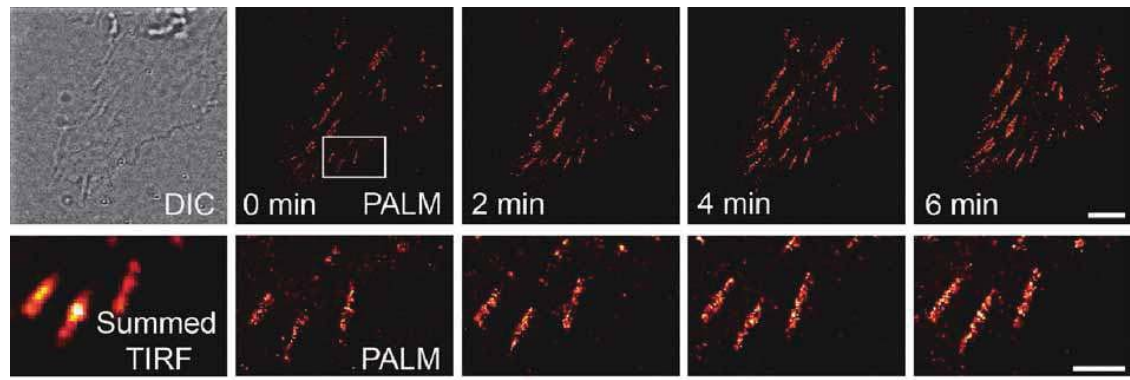

(b)
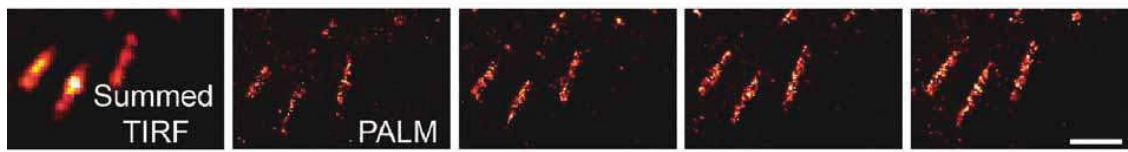

(c)
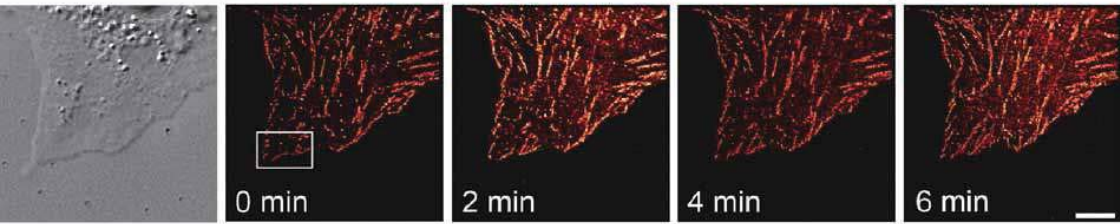

(d)
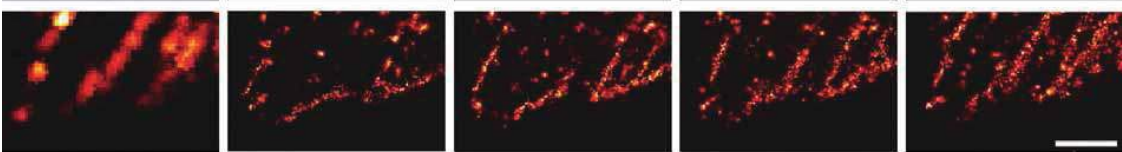

(e)
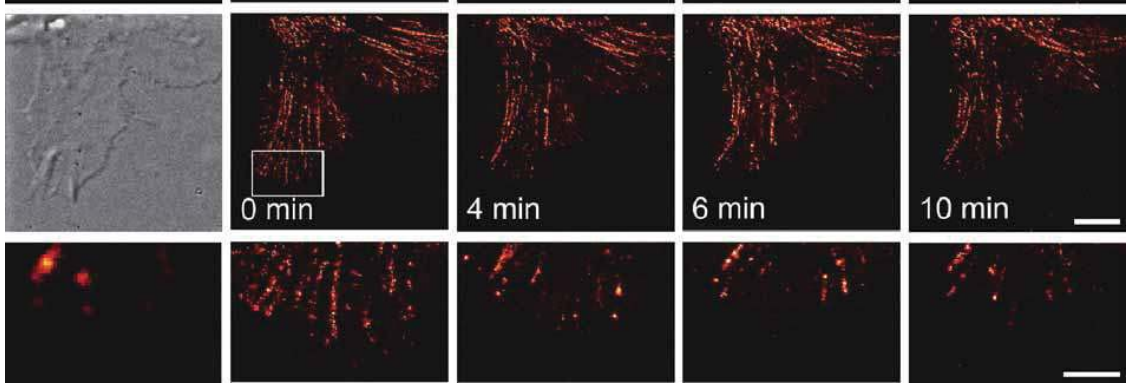

(f)

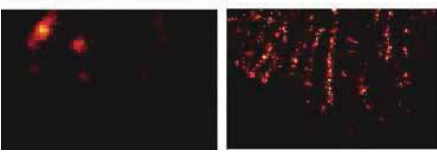

Figure 2.5: Live-cell PALM images of paxillin distributions are shown at (a,c,e) 5 and $(\mathrm{b}, \mathrm{d}, \mathrm{f}) 2 \mu \mathrm{m}$ of scale bar. DIC and TIRF microscopy images are shown at left. The times shown in the images are the final time points of each $30 \mathrm{~s}$ acquisition for (a) and (b) and $60 \mathrm{~s}$ acquisition for the (c) to (f). Reprinted with permission from reference [25]. Copyright 2008 Nature Publishing Group.

responding near-field optical images of the DIP film are shown in Fig. 2.7. The topography image revealed recognized structures of stepped terraces while the near-field optical image showed bright lines and dark regions. The bright features in the optical image (Fig. 2.7 labeled as 2, 3 and 4) were attributed to the DIP domain boundaries. 
(a)

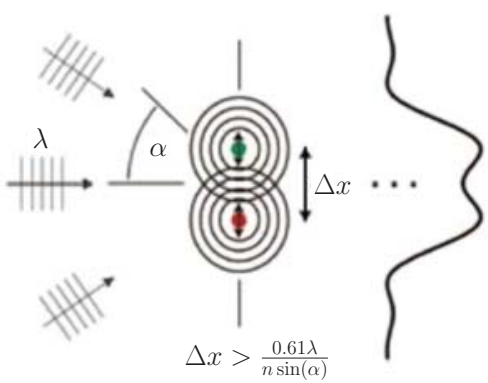

(b)

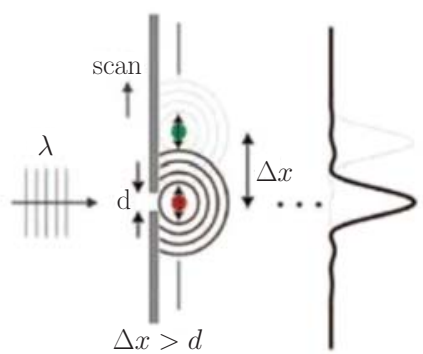

Figure 2.6: Schematic representation of the resolution of (a) conventional optical microscopy and (b) apertured SNOM. The minimum detectable separation of two light scatterers (indicated by the red and the green dots) are shown as $\Delta x$. The resolution for the apertured SNOM is defined by the aperture size (shown as $d$ ) and not by the wavelength of the light. Reprinted with permission from [8]. Copyright 2006 Annual Reviews.

\section{$2.2 \quad$ Imaging ellipsometry}

\subsubsection{Introduction}

Ellipsometry measures the changes of the polarization state of polarized light upon reflection from a surface [33]. If a surface is covered with a thin film (including multiple layer thin films), the whole optical system including the thin film and the substrate influence the polarization state of the light reflected from that surface. Therefore, by measuring the change of the polarization state, it is possible to obtain the optical properties of the layer system such as the film thickness and the refractive index.

The basic measurement parameters in ellipsometry, the amplitude component $\psi$ and phase difference $\Delta$, are related to the complex reflectance ratio $\rho$ of Fresnel reflection coefficients $\left(R_{p}\right.$ and $\left.R_{s}\right)$ for $p$ - and $s$-polarized light $[34,35]$

$$
\rho=\frac{R_{p}}{R_{s}}=\tan (\psi) e^{i \Delta}
$$

These coefficients are complex functions of the angle of incidence $\Phi_{0}$, the wavelength $\lambda$, the optical constants of the substrate $N_{s}$, the ambient medium $n_{0}$, the layers $n_{j}, k_{j}$, and the layer thicknesses $d_{j}$ : 
(a)

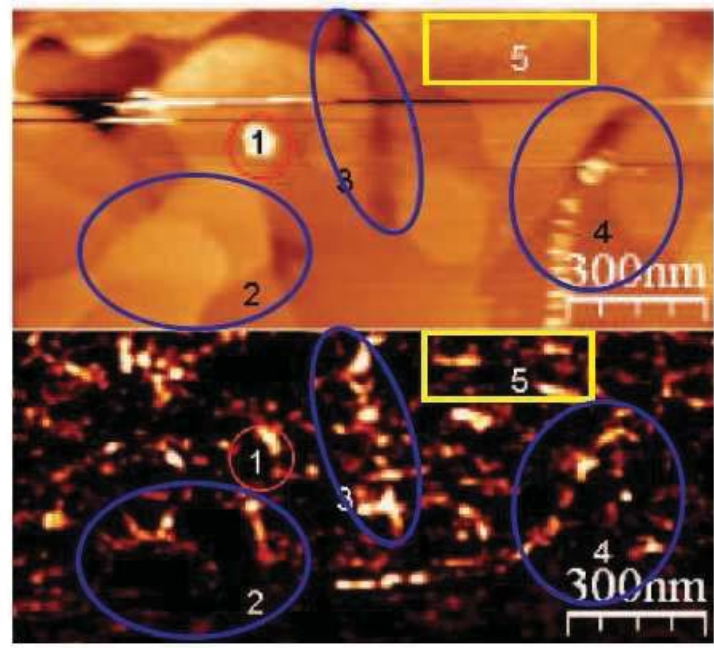

(b)

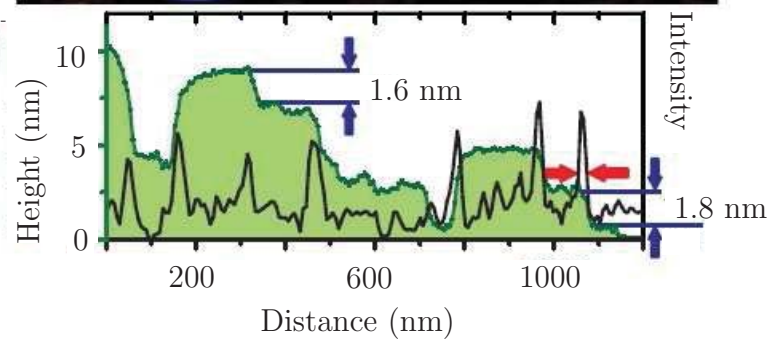

Figure 2.7: Tip enhanced photoluminescence image of a semiconductor film of DIP. In (a), the topography (upper panel) and the optical (lower panel) images are displayed. In (b), the line profile through the topographic image with its correlated optical intensity is shown. Reprinted with permission from [32]. Copyright 2010 The American Physical Society.

$$
\frac{R_{p}}{R_{s}}=F\left(\Phi_{0}, \lambda, N_{s}, n_{0}, n_{j}, k_{j}, d_{j}\right)
$$

where $j$ represents the number of layers [36]. Therefore, the optical properties of the thin film (thickness and the refractive index) cannot be directly determined by the basic measurement parameters (also referred to as ellipsometric angles, $\psi$ and $\Delta$ ) of ellipsometry. The ellipsometric angles must be fitted to an optical model which contains all the parameters given in equation 2.3. Hence, ellipsometry is not a direct method to determine thickness values but it essentially requires optical modeling.

Imaging ellipsometry (IE) combines the sub-nm vertical resolution of ellipsometry with the lateral resolution of optical microscopy $[37,38]$. Therefore, not only 
the ellipsometric quantification but also the visualization of the lateral thickness distribution of thin transparent films on solid substrates are possible in IE. To integrate the imaging capability to an ellipsometer, the instrument is equipped with an objective and a charge-coupled-device (CCD) camera. This combination provides label-free detection and quantification of interface phenomena. This method found widespread applications in a range of research areas such as the investigation of biomolecular interactions [39-44], the imaging of nanometer thick film patterns [35, 45], the detection of graphene flakes [46], and the study of thickness distributions as well as the responsive behavior of thin polymer films [36, $47,48]$.

In addition to its label-free detection capability, IE offers a fast and non-contact measurement. These advantages make IE a primary imaging method to monitor thickness changes and lateral uniformity over large areas [43]. However, drawbacks, particularly associated with the imaging angle, limit the accuracy of IE [37]. The high numerical aperture of the objective, essential to achieve high spatial resolution, cause measurements to be averaged over a changing angle of incidence (AOI). Additionally, the imaging angle causes image distortion and corresponding focus problems. Therefore, IE is exclusively used as a relative measurement technique.

\subsubsection{Instrumentation}

A typical IE consists of a laser, a polarizer and a compensator (also termed quarter-wave plate or retarder) on one side and a long-distance objective, an image scanner, an analyzer and a CCD camera on the other side (Fig. 2.8) [49]. The polarizer converts a beam of light of undefined or mixed polarization into a beam with well-defined polarization. By rotating the polarizer, an unpolarized incident light can be transformed to a beam of linearly polarized light with the direction of polarization corresponding to the angle of rotation axis of the polarizer. The compensator is composed of a birefringent material (such as quartz or mica) with a carefully adjusted thickness such that any linearly polarized light that strikes the plate is divided into two components with different indices of refraction. Depending on the orientation of the quarter-wave plate, it transforms linearly polarized light into elliptically polarized light. When it is set to $45^{\circ}$ with respect to the linear polarization axis, it converts linearly polarized light into circularly polarized light. In IE, the combination of the polarizer and the quarter-wave plate components with their rotatable mounts creates any desired elliptical state of polarization.

If the sample is not depolarizing, the elliptically polarized light reflecting from the sample surface gets converted into linearly polarized light. This linearly polarized beam is detected by a second polarizer, which is used as an analyzer. If the analyzer is set to a $90^{\circ}$ position with respect to the axis of the reflected linearly polarized beam, the light is extinguished. This configuration is termed 


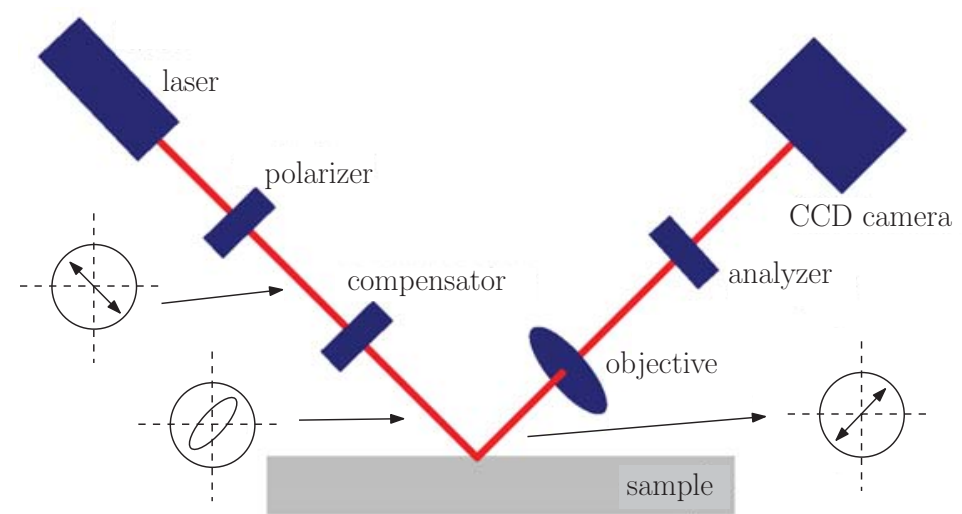

Figure 2.8: Scheme of an imaging ellipsometer. The optical components and the polarization state of the light after passing a certain optical component are shown.

null ellipsometry and it is achieved by finding a minimum of the signal at the detector. The null ellipsometry configuration is often preferred since it allows to cancel any misalignments and other internal system artifacts [37]. By using the null configuration in IE, the signal on one area of the sample surface is minimized (appears black or gray), while the un-nulled areas provide a stronger signal (appears white) at the detector. The selected areas (regions of interest, ROIs) on the sample surface can be measured by the local null-ellipsometry mode and the optical properties can be determined using an appropriate optical model.

An image scanner is used in IE to eliminate depth of focus problems associated with the angle of incidence. This limitation is more pronounced at high magnifications. However, using an image scanner, the objective is continuously moved to record video sequences which are then constructed to an overall sharp image. An uncorrected image of an etched $\mathrm{SiO}_{2}$ film is shown in Fig. 2.9 [37]. This image demonstrates the limitations caused by the angle of incidence and depth of focus: due to the distortion caused by the oblique angle of incidence, the etched squares appear as rectangles. In addition, the top and the bottom parts of the image are defocused due to the limitation of the depth of focus.

Because IE is diffraction limited, the numerical aperture of the microscope objective determines the lateral resolution of IE. The vertical resolution, on the other hand, is in the sub-nanometer range since IE possesses the sensitivity of the ellipsometer. 


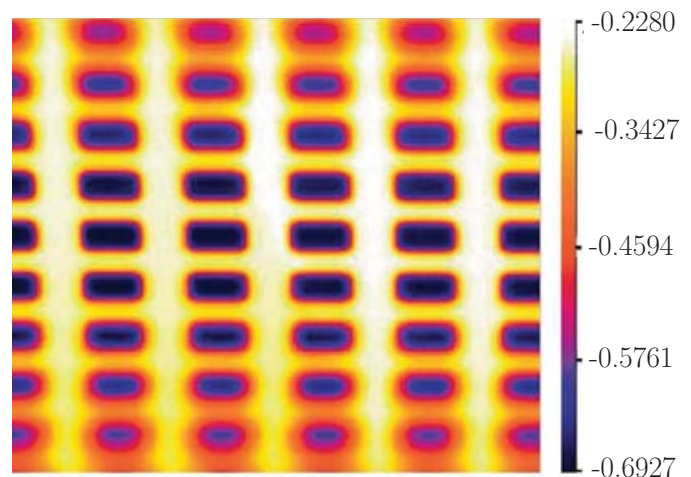

Figure 2.9: Raw IE data of an etched $\mathrm{SiO}_{2}$ film collected at 62.5 degree incident angle. Etched areas are $100 \times 100 \mu \mathrm{m}$ squares. The image resolution is $7 \mu \mathrm{m} /$ pixel. Reprinted with permission from reference [37]. Copyright 2008 Wiley.

\subsubsection{Selected applications of imaging ellipsometry}

In this section, an overview of some relevant applications of IE related to polymer characterization is provided, including combinations of IE with other techniques and spectroscopic imaging ellipsometry. In the first part, the thickness distribution of a polyaniline (PANI) film growth, the lateral heterogeneity of a patterned polypeptide thin film and the thermoresponsive aggregation behavior of a micropatterned hydrogel film determined by IE are discussed. In the second part, techniques in which IE is combined with electrochemical methods and small angle X-ray scattering ( $\mu$ GISAXS) are explained.

\section{Polymer characterization by IE}

In a study by Abrantes et al. IE was used to investigate the thickness distributions of PANI films on gold substrates [47]. Films were synthesized electrochemically under repetitive voltammetric cycling. The thickness distribution of a PANI sample which was grown by 20 cycles on one half of the film and 50 cycles on the other half of the film was imaged by IE (Fig. 2.10(a)). The polymer film which was grown by 20 cycles revealed $17.5 \mathrm{~nm}$ film thickness, while the other half of the film was $43.1 \mathrm{~nm}$ thick. The thickness distributions of the PANI films grown by 20 and 100 cycles showed homogenous layers (Fig. 2.10(b) and (c)). A higher surface roughness was found for thicker polymer films. In conclusion, in this study IE has provided information on the thickness distribution of a polymer film, which cannot be obtained by conventional ellipsometry measurements.

The lateral inhomogeneity of the patterned surface-grafted polypeptide poly- $\gamma$ benzyl-glutamate (PBLG), on a gold surface was studied by IE [48]. Micropatterned areas were prepared by micro-contact printing of initiator molecules (HS- 
(a)

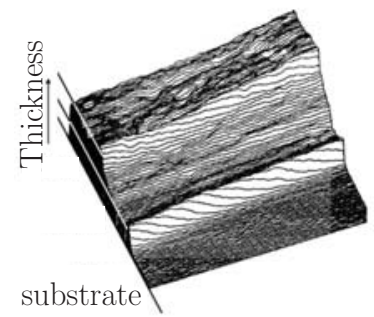

(b)

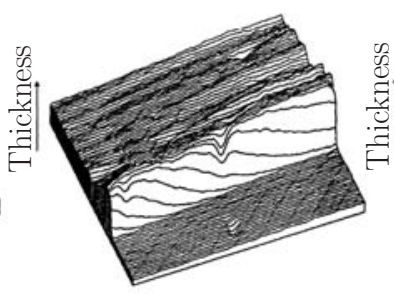

(c)

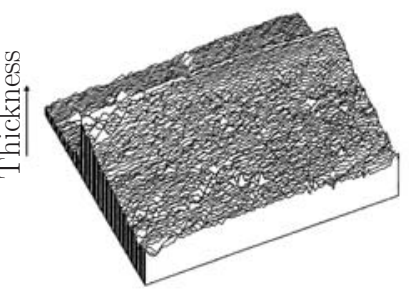

Figure 2.10: Thickness distribution of PANI films grown with (a) half of the film at 20 and the other half at 20+30, (b) 20 and (c) 100 cycles. The images were captured with an imaging ellipsometer and the lateral dimensions of the images are $25 \times 30 \mu \mathrm{m}^{2}$. Reprinted with permission from reference [47]. Copyright 2001 Elsevier.

$(\mathrm{CH})_{n}-\mathrm{NH}_{2}$ ) on a gold substrate. Subsequently the PBLG was prepared by surface initiated ring-opening polymerization of N-carboxyanhydrides of the corresponding amino acids. Fig. 2.11 shows the ellipsometric image of the patterned PBLG film. The thicknesses of the ROIs on the micropatterned polymer film were determined by IE. The dark areas show the polypeptide (approximately $44 \mathrm{~nm}$ thick) and the white areas the gold substrate. Additionally, a loosely absorbed polypeptide layer (approximately $4 \mathrm{~nm}$ thick) was detected on the gold surface.

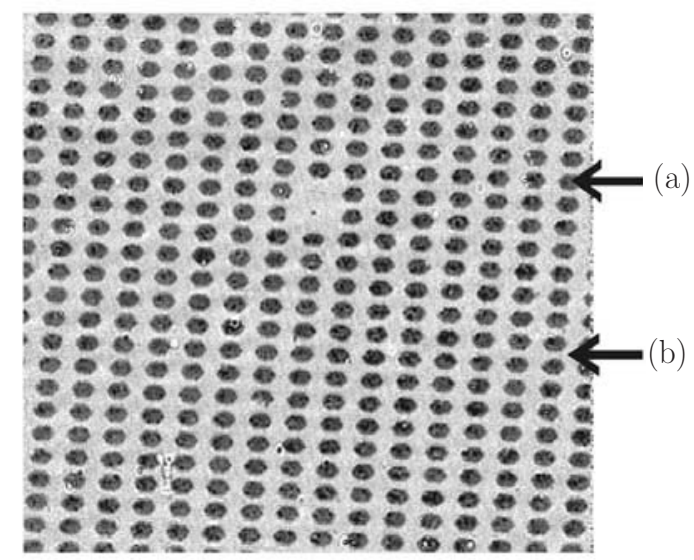

Figure 2.11: Ellipsometric image of the micropatterned polypeptide printed on a gold substrate, arrows depict (a) the polypeptide film and (b) the gold substrate with a approximately $4 \mathrm{~nm}$ thick polypeptide layer. Reprinted with permission from reference [48]. Copyright 2002 Elsevier.

Schmaljohann et al. investigated the thermoresponsive aggregation behavior of a micropattered hydrogel film by in situ by IE [36]. Thin films of a graft polymer, N-isopropylacrylamine-g-poly(ethylene glycol) (PNiPAAm-g-PEG), were cross- 
linked and immobilized on fluoropolymer substrates by low-pressure plasma treatment. A transmission electron microscopy (TEM) grid was used to cover a section of the wafer. The polymer layer which was protected by the TEM grid from plasma exposure was removed by rinsing with chloroform, while the unprotected part was cross-linked and covalently bound to the substrate. The $3 \mathrm{D}$ profile surface map of the micopatterned hydrogel film was recorded with an imaging ellipsometer as shown in Fig. 2.12. It displays the two-dimensional area plot of the micropattern with the ellipsometric parameter $\Delta$ in the third dimension. The decrease of $\Delta$ shown in Fig. 2.12 corresponds to the decrease of the film thickness with increasing temperature. The authors pointed out that the swelling and the collapsing of the hydrogel only occurred in the $z$-direction. This result was attributed to the immobilization process which prevented the swelling of the hydrogel in the $x$ - and $y$-direction. Overall, this study showed that dynamic processes like the swelling and collapsing of a hydrogel can be monitored by IE.

(a)

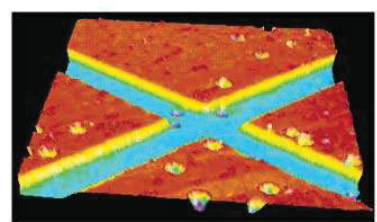

Figure 2.12: Ellipsometric images of the micropatterned hydrogel upon heating at (a) 25 , (b) 30 and (c) $35{ }^{\circ} \mathrm{C}$. The $z$-direction in the $3 \mathrm{D}$ profile represents the ellipsometric data $\Delta$ and the $z$-scale corresponds to $25 \mathrm{~nm}$ decrease in the film thickness. In the micropatterned film, the hydrogels are separated by grooves of $60 \mu \mathrm{m}$ width. Reprinted with permission from reference [36]. Copyright 2005 American Chemical Society.

\section{IE combined methods}

Svoboda et al. used IE in combination with a cyclic voltammetry (CV) based deposition technique to determine film thicknesses and morphologies of polymerized methylene green (poly-MG) films on platinum electrode surfaces [50]. 3D surface morphology obtained simultaneously by in situ IE revealed that the CV deposited polymer films were homogeneous and evenly distributed (Fig. 2.13).

IE in combination with an electrochemical potentiostate was utilized to study adsorption of proteins on charged surfaces (Fig. 2.14(a)) [51]. While the surface potential was controlled electrochemically, protein adsorption was monitored in real time by IE. AC impedance spectroscopy was used to determine the potential of zero charge (PZC) for gold-coated silicon wafers at the solid-liquid interface. Subsequently, the adsorption of fibrinogen was studied on potential controlled (at PZC) and non-biased surfaces by in-situ IE. The graph in Fig. 2.14(c) shows the 
(a)

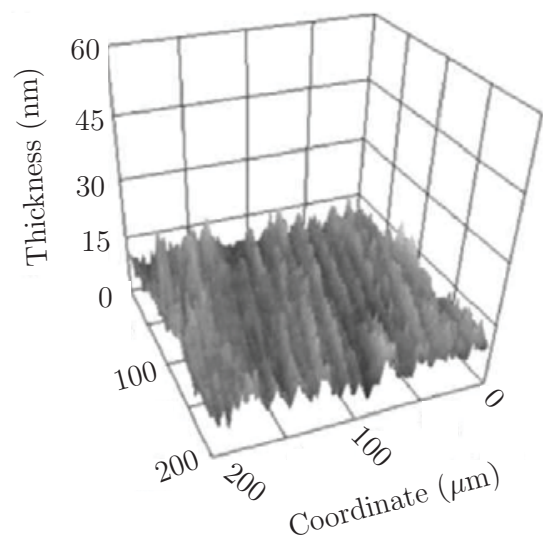

(b)

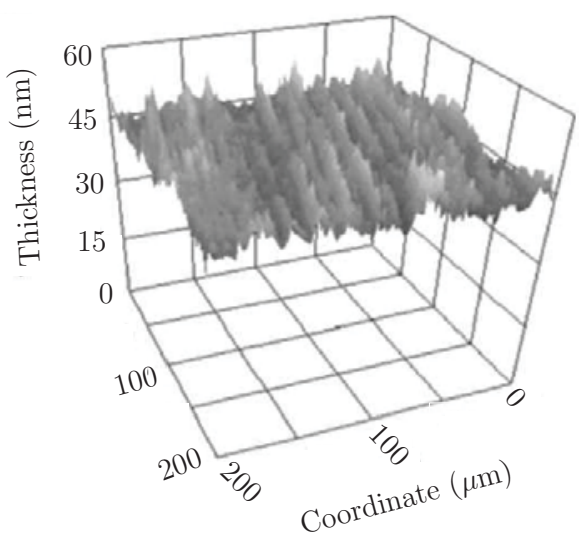

Figure 2.13: Ellipsometric images of a poly-MG film: (a) before and (b) after 16 CV cycles. The images show a $200 \times 200 \mu \mathrm{m}^{2}$ area. Reprinted with permission from reference [50]. Copyright 2007 the Electrochemical Society.

gray scale obtained by IE versus the adsorption time indicating the density of the proteins on the surface. Interestingly, the rate of fibrinogen adsorption on the potential controlled surface was faster than on the non-biased surface. This result was attributed to easier rearrangement of fibrinogen to a more stable position on the uncharged surface than on the charged surface.

More recently, Körstgens et al. proposed a combined IE and microbeam grazing incidence small angle X-ray scattering ( $\mu$ GISAXS) method which additionally enables characterization of structure and morphology [52]. In this combined configuration, IE is utilized to locate and characterize ROIs on a sample and study in situ the influence of external parameters such as temperature and humidity on structures simultaneously with the $\mu$ GISAXS. The self-assembly of colloidal polystyrene (PS) nanospheres on a rough surface, a diblock copolymer island structure generated by dewetting, was investigated simultaneously by $\mu$ GISAXS and IE. The roughness of the surface introduces a chemical heterogeneity in terms of compatibility with the material of the nanospheres.

Figure 2.15 shows the ellipsometric images and the corresponding $2 \mathrm{D} \mu \mathrm{GISAXS}$ patterns. Images on the left indicated with $t<0 \mathrm{~s}$ display the situation before droplet deposition. After placing a sample droplet, the ellipsometric image shows the drop vaguely, while the scattering intensity is very low due to the strong absorption of the incident X-ray beam by the droplet. Frizeau fringes appear in the ellipsometric image due to the thinning of the liquid layer upon evaporation. With further thinning, the layer becomes translucent and the scattering pattern shows increasing intensity close to the direct beam due to the reflection and the refraction of the beam at the droplet-air interface. At $t=2310 \mathrm{~s}$ the system 
(a)

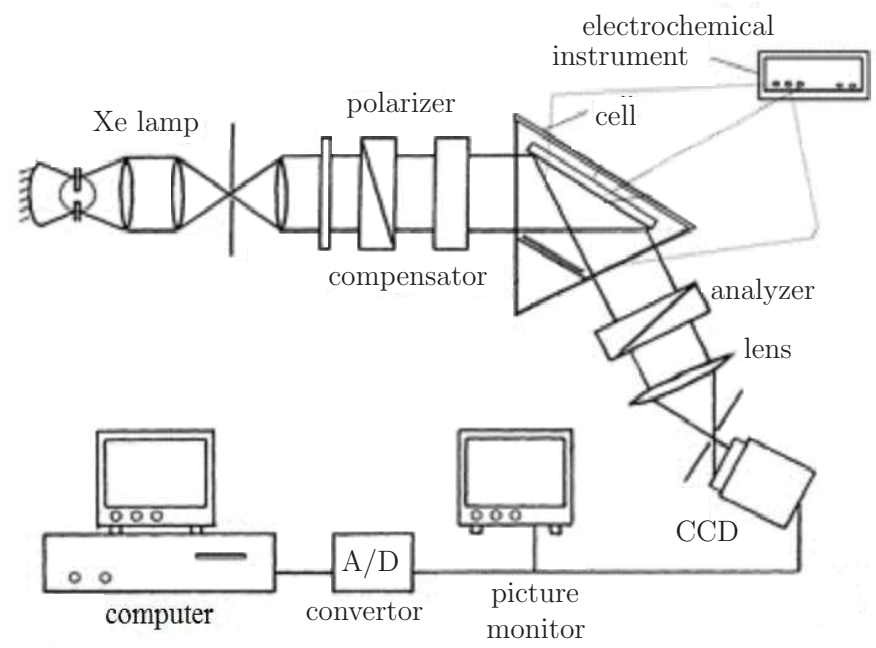

(b) reference

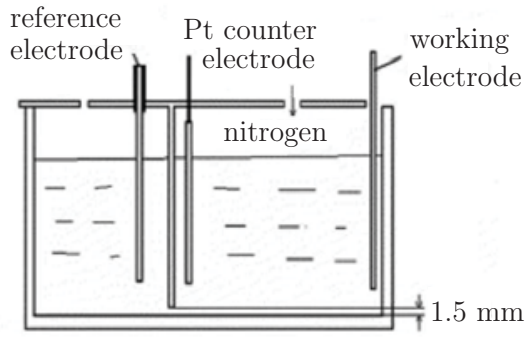

(c)

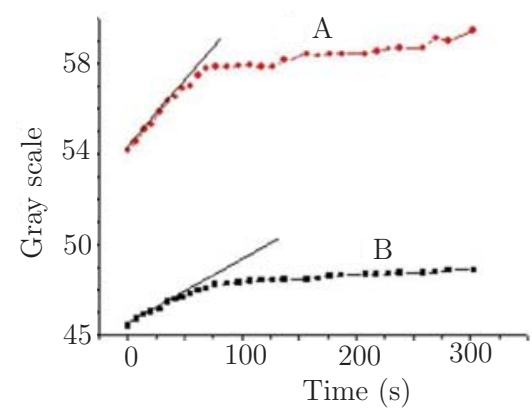

Figure 2.14: In (a) and (b), the schematic diagrams of the IE combined with an electrochemical instrument and the sample cell are shown, respectively. In (c), the graphs of the gray scale which illustrate the surface concentration of fibrinogen are shown versus the adsorption time. The curve A (red) shows the adsorption on the potential controlled surface, while the curve B (black) shows the adsorption on the non-biased surface. Reprinted with permission from reference [51]. Copyright 2004 Elsevier.

reached an equilibrium showing no obvious change in the following scans. Consequently, by combining $\mu$ GISAXS with IE, a full structural and morphological characterization was achieved. 
(a)

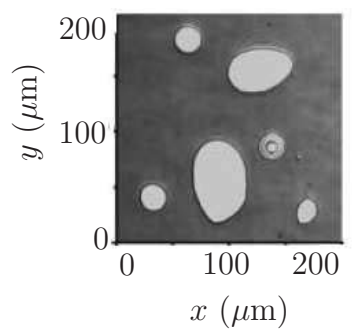

(b)

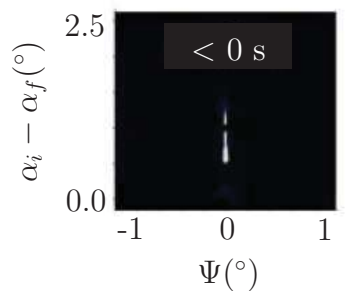

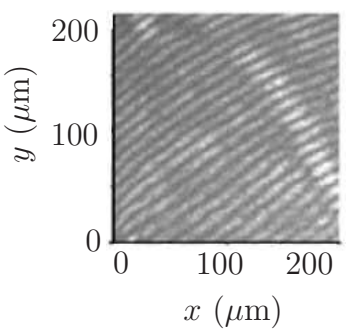

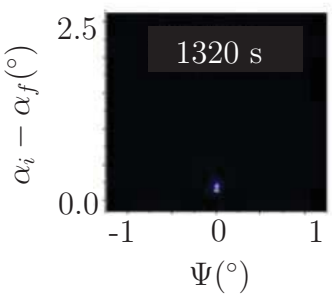

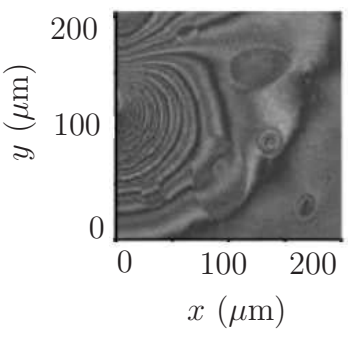

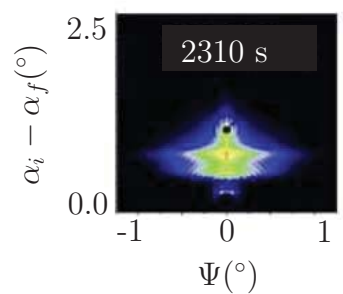

Figure 2.15: Investigation of the self-assembly of colloidal PS nanospheres on a diblock copolymer island structured surface was studied simultaneously with IE and 2D $\mu$ GISAXS. In (a), a series of ellipsometric images and in (b), the corresponding $2 \mathrm{D} \mu$ GISAXS patterns are shown. Data acquisition times are indicated on the top part of the $\mu$ GISAXS patterns. The images at $t<0 \mathrm{~s}$ represent the substrate before droplet deposition and images captured at various times after droplet deposition are shown. See the text for details. Reprinted with permission from reference [52]. Copyright 2009 Springer-Verlag.

\subsection{Scanning near-field optical microscopy}

\subsubsection{Aperture-based near-field optical microscopy}

In aperture-based SNOM, a probe with a small aperture is used to scan the sample in close proximity [53]. Generally, an aperture-based SNOM probe has a sub-wavelength aperture which is formed by tapering an optical fiber and coating its side walls with an aluminum layer to avoid light leakage. The performance of these probes are highly effected by the actual taper shape, the length of the probe and the quality of the aluminum layer.

Neumann et al. have proposed a probe preparation which leads to probes with improved absolute throughput and efficient coupling through the aperture [54]. These probes were prepared first by tapering an optical fiber via heat-pulling procedure and then by coating the fiber with an $220 \mathrm{~nm}$ thick aluminum layer. Next, a focused ion beam (FIB) milling was used to cut the end face of the fiber with a diameter of $370 \mathrm{~nm}$ and the end face coated with a $90 \mathrm{~nm}$ thick gold film. Consequently, a sub-wavelength aperture was milled at the center of the gold 
layer with a diameter of $110 \pm 10 \mathrm{~nm}$. Fig. 2.16(a) and (b) show the schematic and an SEM image of the metal-coated tapered fiber with an aperture in the end face. These probes provide sufficient brightness to image single molecules with an aperture of $45 \mathrm{~nm}$ diameter.

(a)

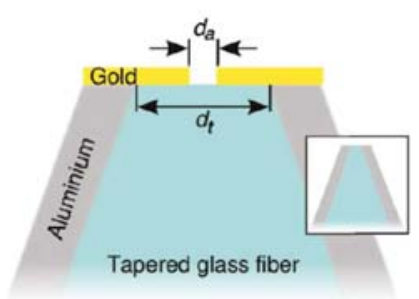

(c)

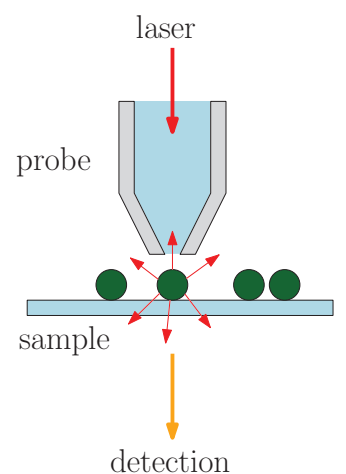

(b)
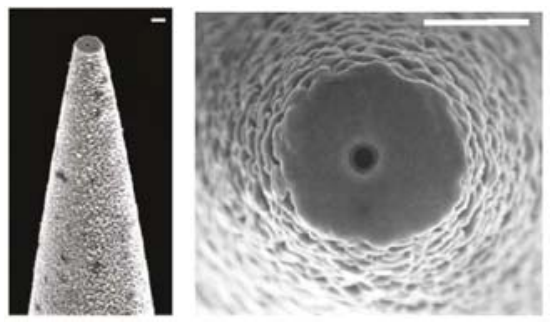

(e) (d)

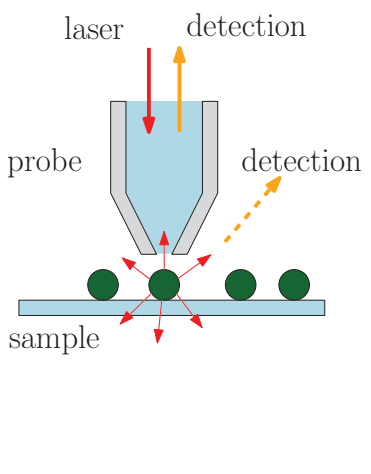

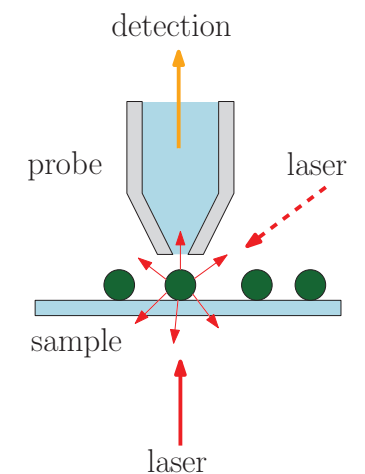

Figure 2.16: Schematic representation and SEM image of an extraordinary optical transmission near-field fiber probe and common configurations of aperture-based SNOM. In (a), the schematic representation of a SNOM fiber with a gold layer that is evaporated on to the end face is shown. The inset depicts the conventional SNOM fiber. The SEM image in (b) show the SNOM fiber probe represented in the scheme. The scale bar is $500 \mathrm{~nm}$. The common configuration (c) illumination, (d) reflection (e) collection modes of aperture-based SNOM are represented. The (a) and (b) are reprinted with permission from reference [54]. Copyright 2010 American Chemical Society.

Common operation modes of aperture-based SNOM techniques are illustrated in Fig. 2.16(c), (d) and (e). The earliest SNOM was operated in the illumination mode, where a dielectric aperture probe illuminates the sample and the light is collected in the far-field (Fig. 2.16(c)) [27]. In the reflection mode, a dielectric probe illuminates the sample and the radiation reflected from the surface is collected either by the probe tip itself or by the far-field optics (Fig. 2.16(d)). However, 
in the collection mode, the dielectric probe collects the light coming through the sample, while the sample is illuminated from the far field (Fig. 2.16(e)).

In aperture-based SNOM, the aperture size is the major factor for achieving high resolution. The intensity of the light at the aperture increases when the diameter of the aperture gets smaller. However, producing such a small aperture in a probe still remains a technical challenge. Mainly, the tapered optical fibers which are fabricated by heating and pulling methods or chemical etching are used as aperture probes. Due to the complication of the production steps, the reproducibility and the quality of the probes are limited.

\subsubsection{Apertureless near-field optical microscopy}

The introduction of apertureless SNOM (a-SNOM), also termed scattering SNOM (s-SNOM), in the mid-1990s has brought simplicity and new possibilities to the technique with the use of already existing atomic force microscopy (AFM) and scanning tunneling microscopy (STM) probes [55-57].

Common a-SNOM configurations are illustrated in Fig. 2.17. In the configuration shown in Fig. 2.17(a), the near-field scattering from the tip is collected by a lens and detected in the far-field [28]. In the scanning plasmon near-field optical microscopy (SPNM) configuration, the illumination is established through total internal reflection (TIR) (Fig. 2.17(b)) [58]. The apertureless probe is then positioned in the evanescent field created by the TIR above the surface. The third a-SNOM configuration, the scanning tunneling optical microscope (STOM), relies on the direct detection of localized fields in the near-field above the sample by uncoated dielectric tips (Fig. 2.17(c)) [59].

(a)

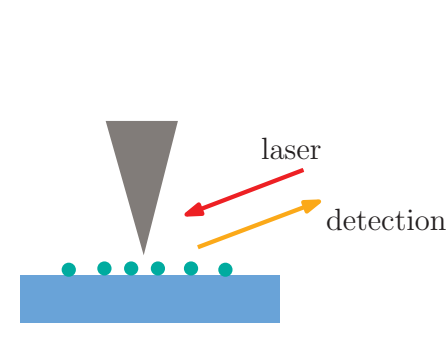

(b)

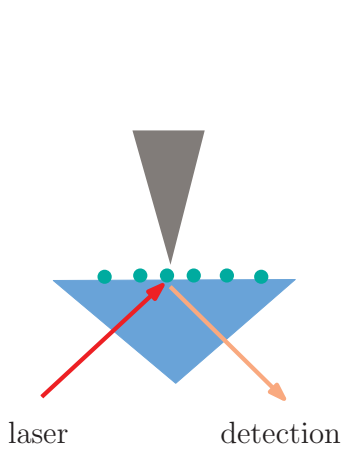

(c)

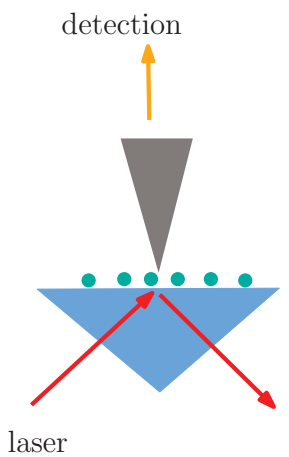

Figure 2.17: Common configurations of apertureless SNOM; (a) scattering type, (b) scattering evanescent excitation mode (c) photon scanning tunneling mode. 
In a-SNOM, the probe can either be used as a local scatterer or a local light source [8]. In the case of a local scatterer, the probe locally perturbs the field at the sample surface and the response to this perturbation is detected in the far field. On the other hand, the probe can also serve as a local light source, since the illumination of the probe can generate a strongly enhanced field near the apex of the probe. In this case, the use of suitable probe materials and the geometry become important to create the proper polarization and excitation conditions.

Various experiments have shown that metal coated probes generate stronger field enhancement and have higher scattering efficiency compared to dielectric or semiconducting tips $[8,55,60]$. Depending on the type of metal, the probe geometry and the illumination parameters such as polarization state and the excitation wavelength, the electromagnetic resonances associated with the free electrons in the metal can significantly enhance the field at the tip apex [8,61]. Therefore, the function of the probes is similar to electromagnetic antennas that convert propagating radiation into confined energy [62].

Although using AFM probes in a-SNOM brings simplicity to the technique, it has the disadvantage of mixing the background scattering with the pure near-field scattering signal. In a-SNOM, AFM provides feedback that keeps the probe in the near field of the surface, which is practically the region within a probe diameter of the sample surface. The scattered light at the near field is then collected in the far field, however, it is mixed with the background scattered light from the tip and the samples [63]. Attempts on eliminating the background scattering focused on demodulating the detector signal at the second or higher harmonic of the tapping frequency $(\Omega)$ owing to the nonlinear dependence of the optical signal on the tip-sample distance [63].

In the following part of this chapter, the emphasis is on tip-enhanced near-field optical microscopy techniques, in particular TERS and SNEM.

\section{Tip-enhanced Raman scattering}

Prior to the discovery of tip enhanced Raman scattering (TERS), surface enhanced Raman spectroscopy (SERS) was used to significantly enhance the sensitivity of Raman spectroscopy $[64,65]$. SERS is a surface sensitive vibrational spectroscopy technique for the structural detection of low concentration components. In SERS, the enhancement of the Raman scattering takes place at a metal surface with nanoscale roughness at which the molecules adsorbed. For SERS enhancement factors of $10^{10}$ to $10^{11}$ have been reported making it suitable for the detection of single molecules [66-69].

There is an ongoing debate in the literature on the exact mechanism of the enhancement effect of SERS. It is usually explained by two theories namely the chemical and the electromagnetic (EM) theory [70]. While the electromagnetic theory explains the excitation via localized surface plasmons, the chemical theory 
proposes the formation of charge-transfer complexes. Hence the chemical theory requires formation of chemical bonds on the surface, while the electromagnetic theory can be applied to cases of physisorbed specimen.

The inhomogeneity of the SERS substrate over the entire sample surface resulted in strong variations in the field enhancement and thus the Raman signal [71, 72]. This obstacle lead to the development of TERS where the enhancing hot spot is reduced to a single particle. Generally in a TERS set-up, an AFM or a STM, which acts as a field enhancing site, and a Raman spectrometer are coupled. In TERS, the tip and the sample are illuminated by an incident laser beam and the Raman scattering is recorded from the focal region [70]. Fig. 2.18 illustrates the enhanced electric field generated in the vicinity of a metallic tip [73]. A metallic probe tip is illumined by an incident beam with appropriate wavelength and polarization state through an objective. An enhanced field is generated at the vicinity of the metallic tip, which is much smaller than the diffraction limited spot. The enhanced field is illustrated in the inset of the Fig. 2.18. The field enhancement mainly occurs due to the excitation of the localized surface plasmon resonances on the metallized probe and the lightening rod effect $[71,74]$. Subsequently, the local Raman signal from the sample at the close vicinity of the metallic tip is detected.

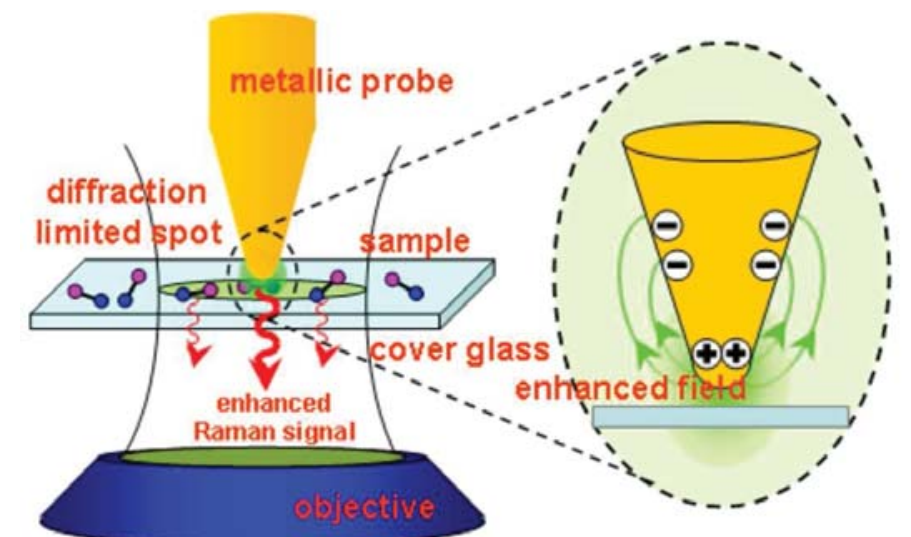

Figure 2.18: Concept of tip-enhanced Raman spectroscopy (TERS) shows a strongly enhanced optical field generated at the apex of sharp metal tip of SPM by the external illumination. Reprinted with permission from reference [73]. Copyright 2011 InTech.

TERS provides chemical and topographical information with a spatial resolution determined by the diameter of the metal tip apex. In an early TERS study by Stöckle et al., a silver coated AFM tip was used to increase the Raman signal of a thin brilliant cresyl blue (BCB) film on a surface [75]. Without the necessity of a special sample preparation, identical enhancement at every location of the sample was achieved contrary to the SERS results. The Raman signal was increased by more than 30 times when the metallized AFM tip was brought into contact with the sample compared to the measurements without the AFM tip (Fig. 2.19). 


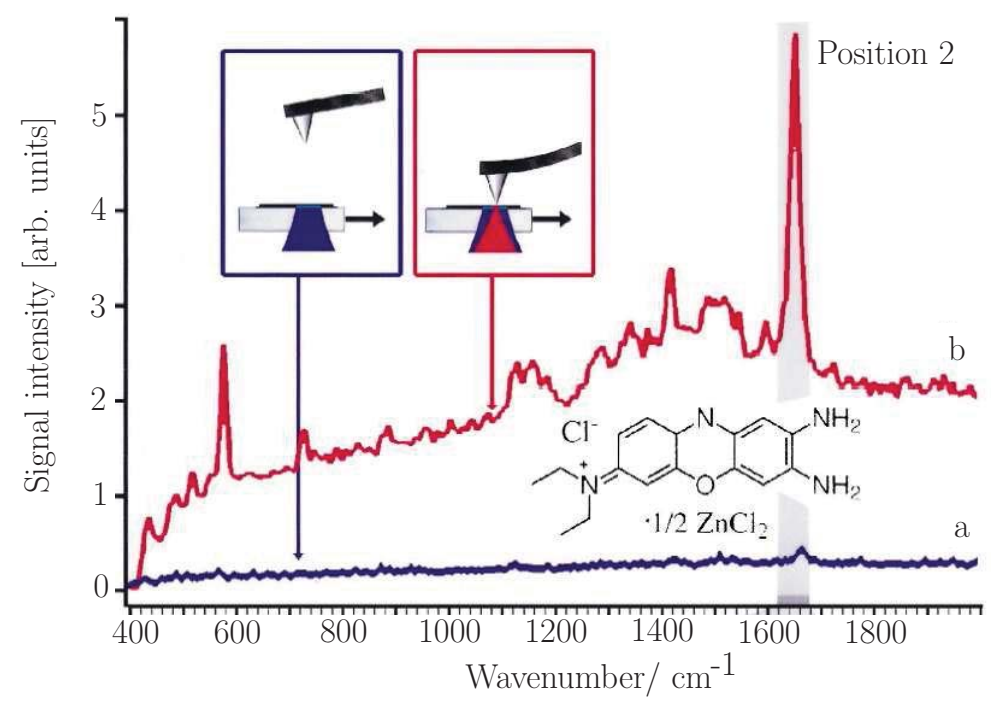

Figure 2.19: Tip-enhanced Raman spectra of brilliant cresyl blue (BCB) when the silver coated SPM tip is (a) away from and (b) in contact with the surface. Reprinted with permission from reference [75]. Copyright 2000 Elsevier.

Tip apex properties such as size, shape and material, play a pivotal role in field enhancement. Dependence of near-field enhancement $\left(\mathrm{E} / \mathrm{E}_{0}\right.$, the ratio between the resulting electromagnetic field with the incident field) on the tip material was calculated using quasistatic approximation [76]. Fig. 2.20 shows the electromagnetic field enhancement simulation results for gold, tungsten, silicon and glass tips of the same size. The gold tip $\left(\varepsilon_{A u}=-9.90+1.05 i\right)$ provided the strongest field enhancement of approximately $E / E_{0}=50$. The tungsten $\left(\varepsilon_{W}=5.05+21.8 i\right)$ and the silicon $\left(\varepsilon_{S i}=12.1+1.04 \times 10^{-8} i\right)$ tips, however, led to much lower field enhancements, in the range of $E / E_{0} \sim 10$. Finally, the glass tip, being a transparent dielectric with small indices of refraction $\left(\varepsilon_{\text {glass }}=2.25\right)$, revealed the weakest field enhancement.

Commonly, to achieve field enhancement in TERS, gold, silver and copper metals are used for probe preparation. Tips are generally prepared by electrochemical etching of solid metals or metal evaporation on conventional Si or SiN AFM tips [71]. The metal evaporation is the most common way to prepare tips for TERS, however, it results in large variability of tips. Therefore, it still remains a challenge to prepare tips with high stability and reproducibility.

In a recent study, thermal oxidization and subsequent metallization of commercial silicon tips was suggested as a method to increase reproducibility of the enhancement effect of TERS tips [77]. Additionally, the metal layer on the tip apex can wear off easily due to abrasion, oxidation and contaminations. Using an ultrathin aluminum oxide $\left(\mathrm{Al}_{2} \mathrm{O}_{3}\right)$ coating (thickness of 2-3 nm) was shown to improve the 


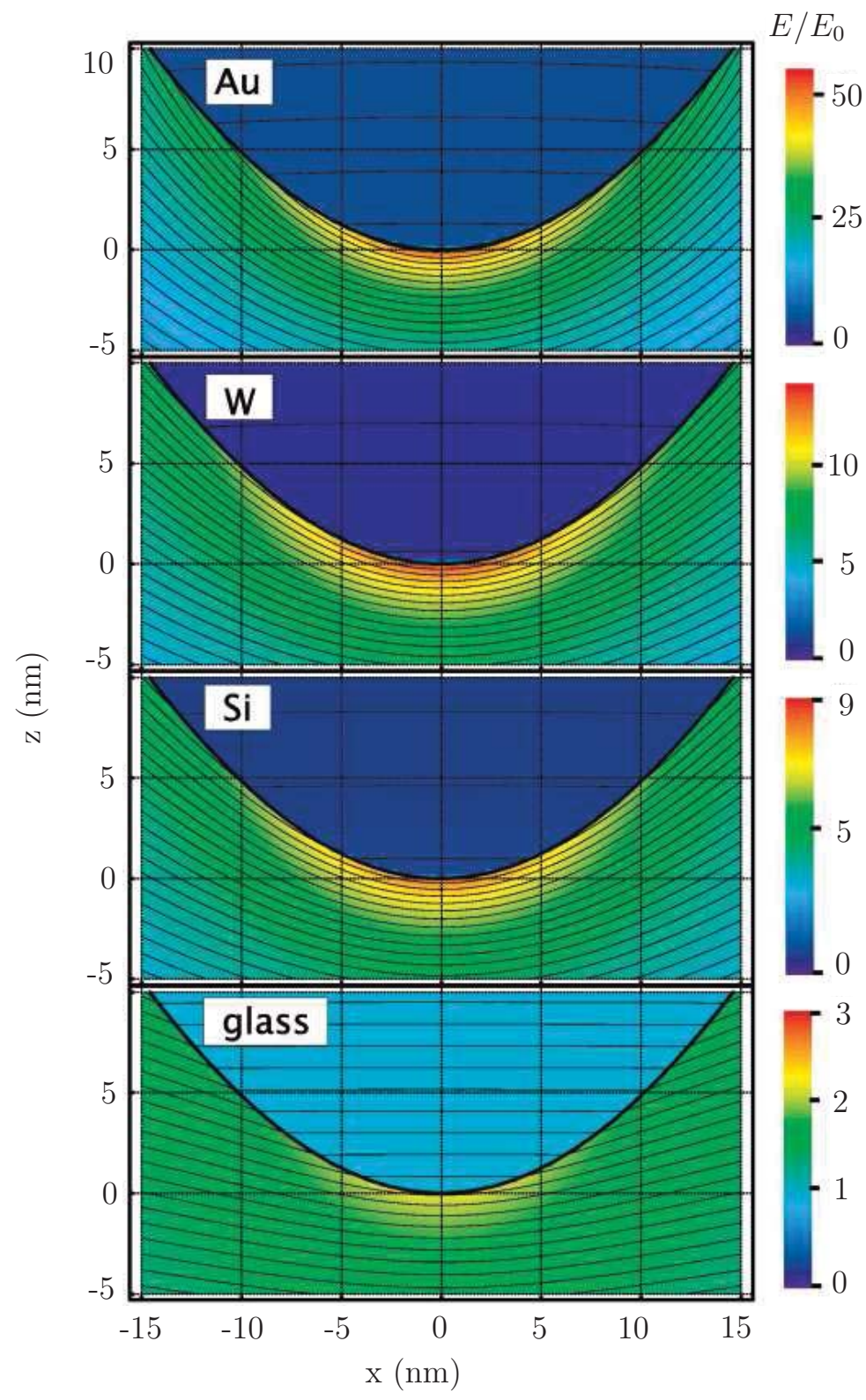

Figure 2.20: Field distribution and corresponding field enhancement $\left(\mathrm{E} / \mathrm{E}_{0}\right)$ for various tip materials namely gold, tungsten, silicon and glass. The tips were free-standing tips with apex radius of $10 \mathrm{~nm}$ and the field enhancement was calculated for the incident field with wavelength $\lambda=630 \mathrm{~nm}$. Reprinted with permission from reference [76]. Copyright 2008 American Chemical Society. 
stability of metal coated tips without sacrificing the initial TERS efficiency [78]. This method helped to increase the tip resistance against mechanical, chemical and possible laser-induced heating damage. It was also proposed that a similar approach could be utilized for improving the stability of other silver-based structures such as SERS substrates and structures for sensor applications.

The applications of TERS imaging in materials and life sciences include a wide range of systems, for instance nanotubes, graphene, solar cell materials, selfassembled monolayers of thiols and mixed supported lipid layers [79-83]. More recently, Lantman et al. have shown that time-resolved tip-enhanced Raman spectroscopy can monitor photocatalytic reactions at the nanoscale as well [84]. A silver coated AFM tip was used to enhance the Raman signal and to simultaneously act as the catalyst. The tip was brought in contact with a self-assembled monolayer of p-nitrothiophenol (pNTP) molecules adsorbed on a substrate of atomically flat gold nanoplates on glass. The photocatalytic reduction process was induced by an excitation light of $532 \mathrm{~nm}$ wavelength while the transformation process during the reaction was monitored by a laser light of $633 \mathrm{~nm}$ wavelength. The TERS spectra showed that the stable and complete pNTP monolayer is transformed into a monolayer with multiple spectral components by the irradiation with the excitation light at $532 \mathrm{~nm}$ (Fig. 2.21). The catalytic activity was proven by the appearance of the $p, \grave{p}$-dimercaptoazobisbenzene (DMAB) peaks at $1440 \mathrm{~cm}^{-1}$ as shown in Fig. 2.21(c).

(a)

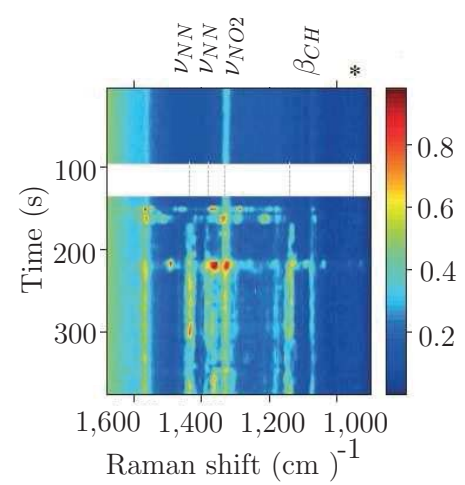

(b)

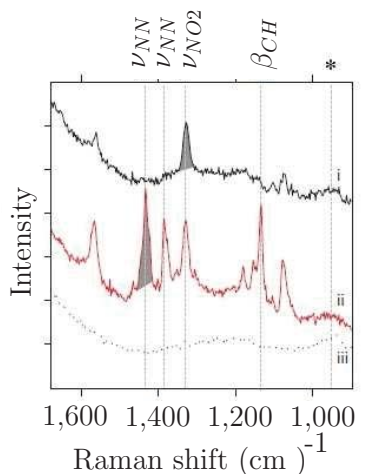

(c)

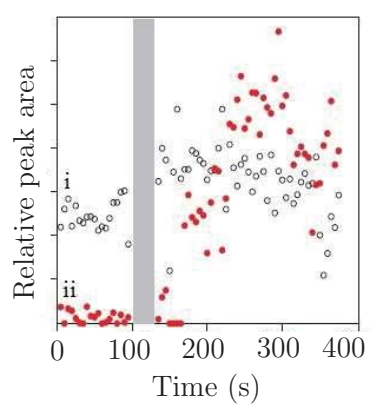

Figure 2.21: TERS measurement data: In (a), TERS spectra before (top) and after (bottom) the illumination at $633 \mathrm{~nm}$ excitation wavelength is shown. In (b) the spectra from (a) shown: (i) $90 \mathrm{~s}$ and (ii) $265 \mathrm{~s}$. the reference spectrum is shown in (iii). In (c), the peak areas as a function of time for the pNTP and DMAB bands are shown at (i) $1335 \mathrm{~cm}^{-1}$ and (ii) $1440 \mathrm{~cm}^{-1}$, respectively. Reprinted with permission from reference [84]. Copyright 2012 Macmillan Publishers Limited. 


\section{Scanning near-field ellipsometric microscopy}

Scanning near-field ellipsometric microscopy (SNEM) was first introduced by Karageorgiev et al. in 2001 [85]. This technique combined an AFM with an ellipsometer to visualize optical inhomogeneities in thin transparent films at a lateral resolution of about $20 \mathrm{~nm}$. In their instrumental arrangement (Fig. 2.22, left), an evanescent field was created at the sample surface by illumination with laser light from below through a prism in total internal reflection (TIR). The resulting evanescent field at the sample surface was then scattered by an AFM tip and collected by the ellipsometer detector enabling optical contrast imaging of inhomogeneities in transparent films. It was stated that the optical contrast was formed due to the interference of the propagating waves generated in the near-field of the tip with the light reflected from the back surface of the film.

A polycrystalline film of a thermotropic liquid crystal was imaged by SNEM to demonstrate the power of the technique (Fig. 2.22, left). The crystallites were revealed in the SNEM optical image as shown in Fig. 2.22(b) and (d). The periodic modulation of the SNEM signal in one monocrystal (Fig. 2.22(b)) was associated with the shape of the crystal (i.e., the thickness) rather than the change in the characteristics of the matter along this crystallite (i.e., refractive index or absorption coefficient). This conclusion was attributed to the fact that the crystalline state gives identical orientation and packing density of the molecules inside one monocrystal.

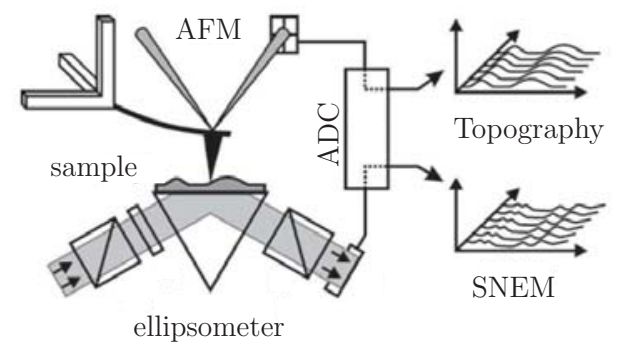

ellipsometer
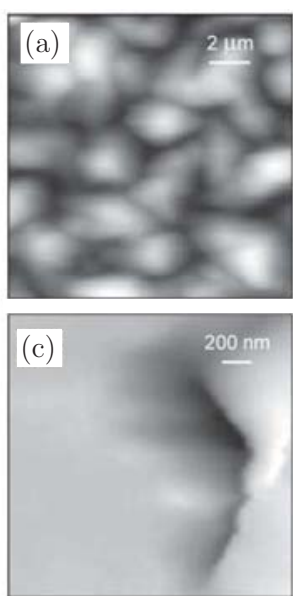
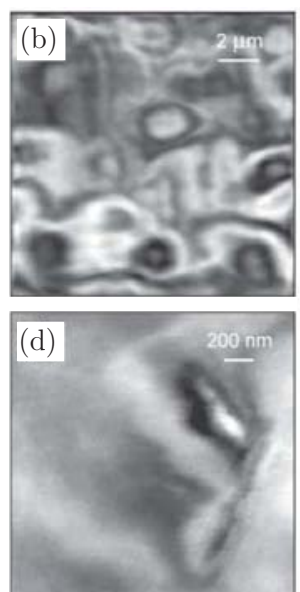

Figure 2.22: On the left side, the instrumental configuration of the AFMellipsometry set-up is depicted. On the right side, (a) and (c) the topography and (b) and (d) the corresponding optical images of a polycrystalline film of a thermotropic liquid crystal are shown. The gray scale denotes 0-1.12 $\mu \mathrm{m}$ (a), 0-265 $\mathrm{mV}$ (b), 0-1.14 $\mu \mathrm{m}$ and 0-42 $\mathrm{mV}$ (d). Reprinted with permission from reference [85]. Copyright 2001 American Institute of Physics. 
A near-field ellipsometry method based on the similar setup but with an aperture SNOM was proposed by Liu et al. in 2010 [86]. In this study, theoretical formulations of the near-field ellipsometry for single layer thin films were provided. Also, the thickness and the refractive index distribution of a thin gold layer coated on a glass slide were calculated. The same authors recently reported a new method based on a reflection configuration [87]. In this new method, a modified dipole-image model was used to analyse the polarization state changes due to the probe-sample interactions and the topography. Reflection configuration enabled characterization of samples with non-transparent or bulky substrates extending the application of SNOM based near-field ellipsometry.

The performance of the system was investigated by experiments with a thin gold film. In these experiments, an $\mathrm{Al}$ coated tapered fiber with an aperture of $50 \mathrm{~nm}$ was used. The system was operated at constant distance mode and a heterodyne detection was used to measure the near-field polarization for $x$ - and $y$ - polarized light at the thin film surface. The experimental characterization including the topography image, calculated thickness and the dielectric constant distributions of a single layer thin film is shown in Fig. 2.23. The thickness distribution was calculated by using the ellipsometry equations (Fig. 2.23(b)). The thickness distribution was found to be correlated to the topography since the sample was prepared by coating a thin layer of gold on a glass substrate.

The calculated average real and imaginary parts of the dielectric constant of the gold film (Fig. 2.23(c) and (d)) was similar to the theoretical value of gold estimated from the Drude model $\left(\varepsilon_{A u}=-11.0592+1.0133 i\right)$. The variations in the dielectric constants were attributed to the porous structure of the film caused by the nano-size air bubbles formed during the coating process. In addition, the resolution was determined from the cross-section along the line $\mathrm{AB}$ in the real part of the dielectric constant distribution image (Fig. 2.23(e)) and found to be around $80 \mathrm{~nm}$. Thus, this near-field ellipsometry method was proposed to be a tool for nano-scale thin film characterization.

An alternative SNEM configuration was introduced by Tranchida et al. in 2011 in our laboratory [88]. In this approach variations in the polarization state of the electromagnetic field at the apex of a metallic AFM tip placed in the proximity of the sample surface were recorded. Instrumental details and the contrast mechanism of this configuration differed from the set-up proposed by Karageorgiev et al. [85]. Our set-up was operated at an incident angle away from the critical angle, whereas in the configuration proposed by Karageorgiev et al., the sample was illuminated under TIR. This detail changes the origin of the field enhancement.

Local field enhancement, in the configuration proposed by Tranchida et al., occurred at the vicinity of the metallic tip due to the confinement of the surface charge density at the tip apex, the so-called "lightning-rod effect", and the localized surface plasmon excitation. In contrast, in the configuration proposed by Karageorgiev et al., the metallic tip is scanned within the evanescent field on the sample surface. The set up proposed by Tranchida et al. was operated in 
(a)

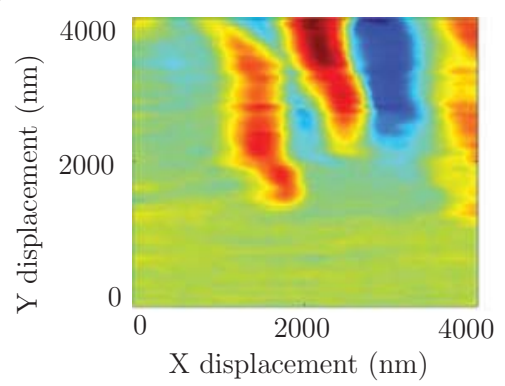

(c)

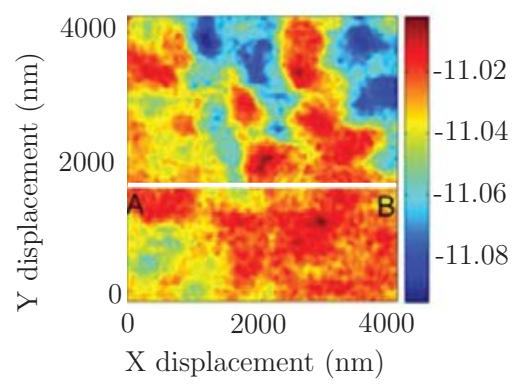

(b)

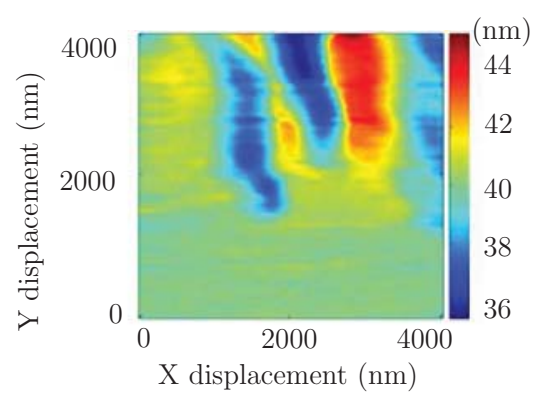

(d)

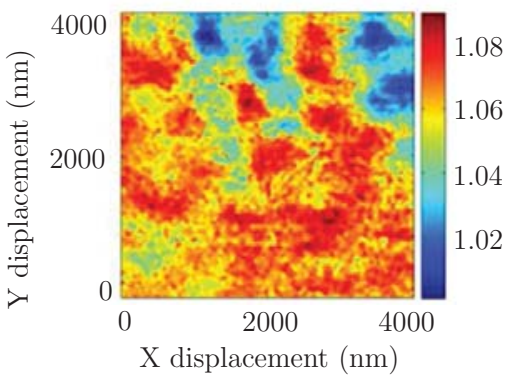

(e)

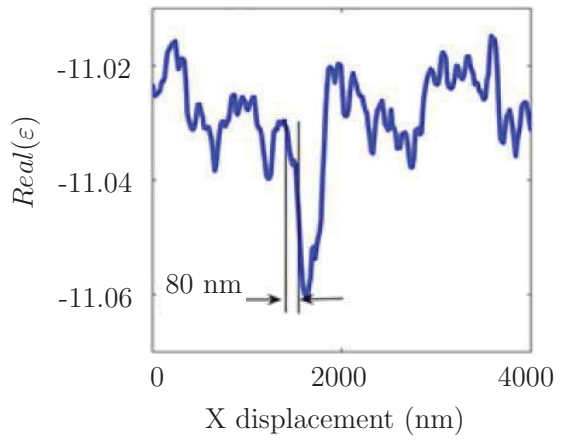

Figure 2.23: SNEM characterization of a thin gold film. In (a), the topography and in (b), the thickness data calculated from the simulations using ellipsometry equations are displayed. In (c) and (d), the real and the imaginary parts of the calculated dielectric constants are shown, respectively. Areas in the dielectric constant map, which show variations from the topography is related to the porosity of the sample. In (e), the cross-section of the real part of the dielectric constant along the line $\mathrm{AB}$ is depicted. Reprinted with permission from reference [87]. Copyright 2012 Elsevier.

contact mode AFM and the optical signal was recorded in null-ellipsometry conditions. The overall optical signal (I) was suggested to be composed of three parts, namely a constant background at a fixed vertical position of the tip ( $\mathrm{I}_{\text {background }}$ ), the near-field signal $\left(\mathrm{I}_{1}\right)$ and the signal due to the vertical displacement of the tip 
$\left(I_{0}\right)$ [88]. The dependence of the value of $\mathrm{I}(\mathrm{x}, \mathrm{y}, \mathrm{z})$ on $\delta \mathrm{z}$ (where $z$ is the sample normal direction) was determined by recording the optical signal, while performing a force-displacement measurement on glass. The background intensity- which follows a sinusoidal function-subtracted intensity plot versus the tip-sample separation is shown in Fig. 2.24. The corrected intensity decays steeply with increasing tip-sample distance and reaches the noise level of the detector at distances around $100 \mathrm{~nm}$ (see Fig. 2.24).

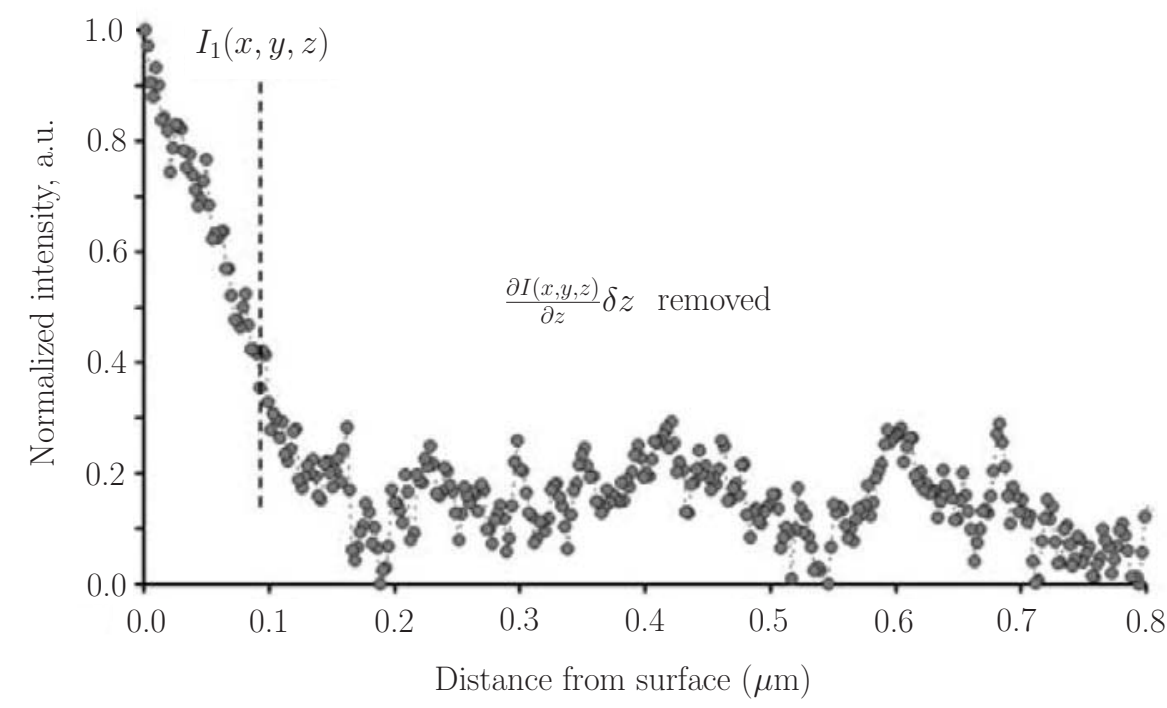

Figure 2.24: Intensity versus distance curve corrected for the effect of background and interference. Reprinted with permission from reference [88]. Copyright 2011 The Royal Society of Chemistry.

SNEM optical and AFM topography images of a poly (methyl methacrylate) (PMMA) thin film loaded with $80 \mathrm{~nm}$ diameter silver nanoparticles were simultaneously captured by SNEM (Fig. 2.25) [88]. The silver nanoparticles were clearly identified both, in contact mode AFM height and the corresponding optical images. The contrast in the optical image was attributed to the difference among the particles and the polymer in scattering the incident light.

Overall, the different SNEM configurations have demonstrated the great potential of the technique for thin film characterization. Using new models to define ellipsometry equations that consider the near-field sample probe interactions and the topography of the thin films have shown the capability of near-field ellipsometry in determining the thickness and dielectric constant distributions of thin films with nanometer scale resolution [87]. Moreover, recording the changes in the state of the polarization of the light scattered at the close vicinity of the tipsample coupled system have shown the capability of using AFM in combination with ellipsometry to obtain near-field optical images [85, 88]. 


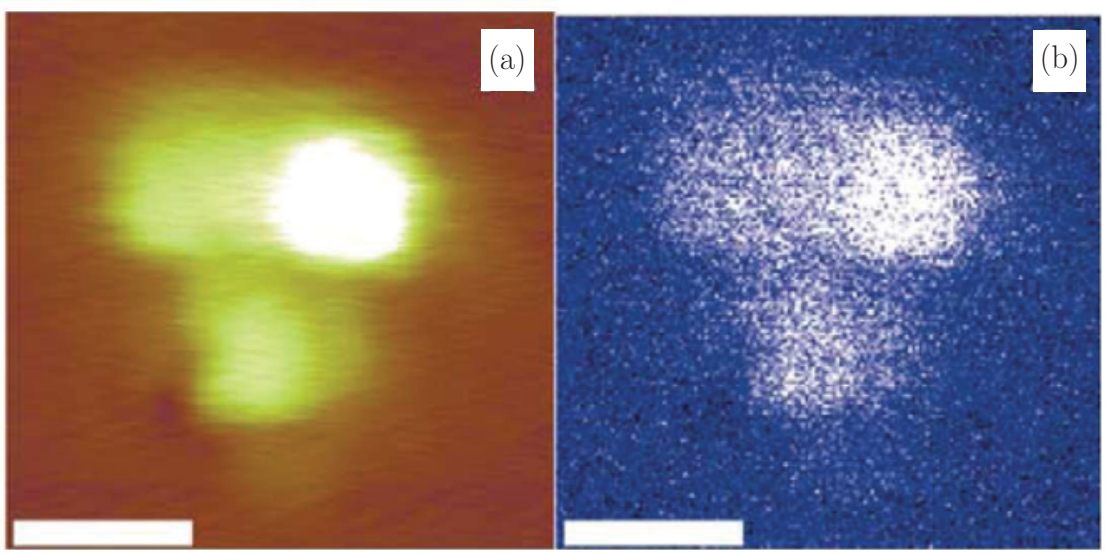

Figure 2.25: SNEM images of a PMMA film loaded with silver nanoparticles, (A) AFM topography image (the $z$-scale covers $40 \mathrm{~nm}$ ) and (B) optical image (the $z$-scale covers $40 \mathrm{mV}$ ). The images were captured by a gold-coated tip at a scan speed of $280 \mathrm{~nm} \mathrm{~s}^{-1}$. The scale bar represents $100 \mathrm{~nm}$. Reprinted with permission from reference [88]. Copyright 2011 The Royal Society of Chemistry.

\subsection{Conclusions}

In this chapter, fundamentals and selected applications of various microscopy techniques that evolved into enabling platforms for nanoscale characterization providing resolution below the diffraction limit were discussed. Firstly, the emphasis was on high spatial resolution fluorescence microscopy techniques including SIM, STED, PALM and STORM which, for instance, enable the investigation of dynamic processes of biomolecules in living cells or reveal nanoscopic features of cellular structures.

Later, the basics and applications of IE, a diffraction limited optical microscopy that enables ellipsometric quantification as well as visualization of the lateral thickness distribution of thin transparent films on solid substrates, were discussed. IE enables the investigation of lateral inhomogeneity of patterned surfaces and dynamic studies like thermoresponsive aggregation behavior of a micropatterned hydrogel film. Combining IE with other techniques for instance electrochemical methods enables studies of dynamic processes such as protein adsorption on charged surfaces or film deposition.

Finally, SNOM techniques such as TERS and SNEM were considered. TERS allows one to obtain local spectroscopic information in various materials even yet brings up the possibility of monitoring photocatalytic reactions at the nanoscale. SNEM facilitates the nanoscale thin film characterization of materials such as heterogeneous thin films and polycrystalline films of thermotropic liquid crystals. 


\section{Bibliography}

[1] Spencer, M. Fundamentals of Light Microscopy; Cambridge University Press: New York, 1982.

[2] Murphy, D. B.; Davidson, M. W. Fundamentals of Light Microscopy and Electronic Imaging; Wiley-Blackwell: Hoboken, N.J., 2013.

[3] Abramowitz, A.; Davidson, M. W.; Anatomy of the microscope: Introduction; Website; 2014; http://www.olympusmicro.com/primer/anatomy/introduction. html.

[4] Schatten, H. Micron 2011, 42, 175-185.

[5] Müller, E. W. Science 1965, 149, 591-601.

[6] Firtel, M.; Beveridge, T. Micron 1995, 26, 347-362.

[7] Novotny, L.; Hecht, B. Principles of Nano-Optics; Cambridge University Press: New York, 2008.

[8] Novotny, L.; Stranick, S. J. Annu. Rev. Phys. Chem. 2006, 57, 303-331.

[9] Abbe, E. Archiv fur Mikroskopische Anatomie 1873, 9, 413-468.

[10] Evanko, D. Nat. Methods 2009, 6, 19-20.

[11] Günzler, H.; Williams, E. Handbook of Analytical Techniques; Wiley VCH, 2001.

[12] Hell, S. W.; Wichmann, J. Opt. Lett. 1994, 19, 780-782.

[13] Hell, S. W. Nat. Methods 2009, 6, 24-32.

[14] Hell, S. W. Science 2007, 316, 1153-1158.

[15] Best, G.; Amberger, R.; Baddeley, D.; Ach, T.; Dithmar, S.; Heintzmann, R.; Cremer, C. Micron 2011, 42, 330-335.

[16] Busko, D.; Baluschev, S.; Crespy, D.; Turshatov, A.; Landfester, K. Micron 2012, 43, 583-588.

[17] Brown, T. A.; Fetter, R. D.; Tkachuk, A. N.; Clayton, D. A. Methods 2010, 51, 458-463.

[18] Rust, M. J.; Bates, M.; Zhuang, X. W. Nat. Methods 2006, 3, 793-795.

[19] Shao, L.; Isaac, B.; Uzawa, S.; Agard, D. A.; Sedat, J. W.; Gustafsson, M. G. L. Biophys. J. 2008, 94, 4971-4983.

[20] Rittweger, E.; Rankin, B. R.; Westphal, V.; Hell, S. W. Chem. Phys. Lett. 2007, 442, 483-487. 
[21] Hess, S. T.; Girirajan, T. P. K.; Mason, M. D. Biophys. J. 2006, 91, 42584272 .

[22] Bates, M.; Huang, B.; Zhuang, X. W. Curr. Opin. Chem. Biol. 2008, 12, 505-514.

[23] Eggeling, C.; Ringemann, C.; Medda, R.; Schwarzmann, G.; Sandhoff, K.; Polyakova, S.; Belov, V. N.; Hein, B.; von Middendorff, C.; Schönle, A.; Hell, S. W. Nature 2009, 457, 1159-U121.

[24] Huang, B.; Wang, W. Q.; Bates, M.; Zhuang, X. W. Science 2008, 319, 810-813.

[25] Shroff, H.; Galbraith, C. G.; Galbraith, J. A.; Betzig, E. Nat. Methods 2008, 5, 417-423.

[26] Mauser, N.; Hartschuh, A. Chem. Soc. Rev. 2014, 43, 1248-1262.

[27] Heinzelmann, H.; Pohl, D. W. Appl. Phys. a-Mater. 1994, 59, 89-101.

[28] Kirstein, S. Curr. Opin. Colloid. In. 1999, 4, 256-264.

[29] Synge, E. H. Philos. Mag. 1928, 6, 356-362.

[30] Pohl, D. W.; Denk, W.; Lanz, M. Appl. Phys. Lett. 1984, 44, 651-653.

[31] Lewis, A.; Isaacson, M.; Harootunian, A.; Muray, A. Ultramicroscopy 1984, 13, 227-231.

[32] Zhang, D.; Heinemeyer, U.; Stanciu, C.; Sackrow, M.; Braun, K.; Hennemann, L. E.; Wang, X.; Scholz, R.; Schreiber, F.; Meixner, A. J. Phys. Rev. Lett. 2010, 104, 56601 1-4.

[33] Azzam, R. M. A.; Bashara, N. M. Ellipsometry and Polarized Light; NorthHolland Pub. Co.: Amsterdam, 1987.

[34] Hennig, A.; Eichhorn, K. J.; Staudinger, U.; Sahre, K.; Rogalli, M.; Stamm, M.; Neumann, A. W.; Grundke, K. Langmuir 2004, 104, 6685-6691.

[35] Neumaier, K. R.; Elender, G.; Sackmann, E.; Merkel, R. Europhys. Lett. 2000, 49, 14-19.

[36] Schmaljohann, D.; Nitschke, M.; Schulze, R.; Eing, A.; Werner, C.; Eichhorn, Y. J. Langmuir 2005, 21, 2317-2322.

[37] Asinovski, L.; Beaglehole, D.; Clarkson, M. T. Phys. Status Solidi A 2008, 205, 764-771.

[38] Jin, G.; Jansson, R.; Arwin, H. Rev. Sci. Instrum. 1996, 67, 2930-2936.

[39] Bae, Y. M.; Oh, B. K.; Lee, W.; Lee, W. H.; Choi, J. W. Anal. Chem. 2004, 76, 1799-1803. 
[40] Huang, C. H.; Li, J. X.; Tang, Y.; Wang, C. X.; Hou, C. J.; Huo, D. Q.; Chen, Y. Y.; Jin, G. Mat. Sci. Eng. C-Mater. 2011, 31, 1609-1613.

[41] Jin, G.; Meng, Y. H.; Liu, L.; Niu, Y.; Chen, S.; Cai, Q.; Jiang, T. J. Thin Solid Films 2011, 519, 2750-2757.

[42] Bae, Y. M.; Oh, B. K.; Lee, W.; Lee, W. H.; Choi, J. W. Biosens. Bioelectron 2004, 20, 895-902.

[43] Faiss, S.; Schuy, S.; Weiskopf, D.; Steinem, C.; Janshoff, A. J. Phys. Chem. $B$ 2007, 111, 13979-13986.

[44] Howland, M. C.; Szmodis, A. W.; Sanii, B.; Parikh, A. N. Biophys. J. 2007, 92, 1306-1317.

[45] Rotermund, H. H.; Haas, G.; Franz, R. U.; Tromp, R. M.; Ertl, G. Science 1995, 270, 608-610.

[46] Wurstbauer, U.; Roling, C.; Wurstbauer, U.; Wegscheider, W.; Vaupel, M.; Thiesen, P. H.; Weiss, D. App. Phys. Lett. 2010, 97, 231901 1-3.

[47] Abrantes, L. M.; Correia, J. P.; Savic, M.; Jin, G. Electrochim. Acta. 2001, 46, 3181-3187.

[48] Beyerlein, D.; Kratzmller, T.; Eichhorn, K. J. Vib. Spectrosc. 2002, 29, 223 227.

[49] Pak, H. K.; Law, B. M. Rev. Sci. Instrum. 1995, 66, 4972-4976.

[50] Svoboda, V.; Cooney, M. J.; Rippolz, C.; Liaw, B. Y. J. Electrochem. Soc. 2007, 154, D113-D116.

[51] Yu, Y.; Jin, G. J. Colloid Interf. Sci. 2005, 283, 477-481.

[52] Körstgens, V.; Wiedersich, J.; Meier, R.; Perlich, J.; Roth, S. V.; Gehrke, R.; Müller-Buschbaum, P. Anal. Bioanal. Chem. 2010, 396, 139-149.

[53] Hecht, B.; Sick, B.; Wild, U. P.; Deckert, V.; Zenobi, R.; Martin, O. J. F.; Pohl, D. W. J. Chem. Phys. 2000, 112, 7761-7774.

[54] Neumann, L.; Pang, Y.; Houyou, A.; Juan, M. L.; Gordon, R.; van Hulst, N. F. Nano Lett. 2011, 11, 355-360.

[55] Inouye, Y.; Kawata, S. Opt. Lett. 1994, 19, 159-161.

[56] Zenhausern, F.; O’boyle, M. P.; Wickramasinghe, H. K. Appl. Phys. Lett. 1994, 65, 1623-1625.

[57] Bachelot, R.; Gleyzes, P.; Boccara, A. C. Opt. Lett. 1995, 20, 1924-1926.

[58] Dehollander, R. B. G.; Vanhulst, N. F.; Kooyman, R. P. H. Ultramicroscopy 1995, 57, 263-269. 
[59] Vigoureux, J. M.; Girard, C.; Courjon, D. Opt. Lett. 1989, 14, 1039-1041.

[60] Novotny, L.; Sanchez, E. J.; Xie, X. S. Ultramicroscopy 1998, 71, 21-29.

[61] Zayats, A. V. Opt. Commun. 1999, 161, 156-162.

[62] Novotny, L.; van Hulst, N. Nat. Photonics 2011, 5, 83-90.

[63] Keilmann, F.; Hillenbrand, R. Phil. Trans. R. Soc. Lond. A 2004, 362, 787805.

[64] Sharma, B.; Frontiera, R. R.; Henry, A. I.; Ringe, E.; Van Duyne, R. P. Mater. Today 2012, 15, 16-25.

[65] Stiles, P. L.; Dieringer, J. A.; Shah, N. C.; Van Duyne, R. R. Annu. Rev. Anal. Chem. 2008, 1, 601-626.

[66] Blackie, E. J.; Le Ru, E. C.; Etchegoin, P. G. J. Am. Chem. Soc. 2009, 131, 14466-14472.

[67] Le Ru, E. C.; Blackie, E.; Meyer, M.; Etchegoin, P. G. J. Phys. Chem. C 2007, 111, 13794-13803.

[68] Nie, S. M.; Emery, S. R. Science 1997, 275, 1102-1106.

[69] Le Ru, E. C.; Meyer, M.; Etchegoin, P. G. J. Phys. Chem. B 2006, 110, 1944-1948.

[70] Pettinger, B.; Schambach, P.; Villagomez, C. J.; Scott, N. Annu. Rev. Phys. Chem. 2012, 63, 379-399.

[71] Bailo, E.; Deckert, V. Chem. Soc. Rev. 2008, 37, 921-930.

[72] Deckert-Gaudig, T.; Deckert, V. Phys. Chem. Chem. Phys. 2010, 12, 1204012049.

[73] Wang, J.; Wu, X.; Wang, R.; Zhang, M. Electronic Properties of Carbon Nanotubes; InTech, 2011.

[74] Asghari-Khiavi, M.; Wood, B. R.; Hojati-Talemi, P.; Downes, A.; McNaughton, D.; Mechler, A. J. Raman Spectrosc. 2012, 43, 173-180.

[75] Stöckle, R. M.; Suh, Y. D.; Deckert, V.; Zenobi, R. Chem. Phys. Lett. 2000, 318, 131-136.

[76] Behr, N.; Raschke, M. B. J. Phys. Chem. C 2008, 112, 3766-3773.

[77] Hayazawa, N.; Yano, T.; Kawata, S. J. Raman Spectrosc. 2012, 43, 11771182.

[78] Barrios, C. A.; Malkovskiy, A. V.; Kisliuk, A. M.; Sokolov, A. P.; Foster, M. D. J. Phys. Chem. C 2009, 113, 8158-8161. 
[79] Yano, T.; Verma, P.; Saito, Y.; Ichimura, T.; Kawata, S. Nat. Photonics 2009, 3, 473-477.

[80] Saito, Y.; Verma, P.; Masui, K.; Inouye, Y.; Kawata, S. J. Raman Spectrosc. 2009, 40, 1434-1440.

[81] Zhang, D.; Wang, X.; Braun, K.; Egelhaaf, H. J.; Fleischer, M.; Hennemann, L.; Hintz, H.; Stanciu, C.; Brabec, C. J.; Kern, D. P.; Meixner, A. J. J. Raman Spectrosc. 2009, 40, 1371-1376.

[82] Stadler, J.; Schmid, T.; Opilik, L.; Kuhn, P.; Dittrich, P. S.; Zenobi, R. Beilstein J. Nanotech. 2011, 2, 509-515.

[83] Opilik, L.; Bauer, T.; Schmid, T.; Stadler, J.; Zenobi, R. Phys. Chem. Chem. Phys. 2011, 13, 9978-9981.

[84] Lantman, E. M. S.; Deckert-gaudig, T.; Mank, A. J. G.; Deckert, V.; Weckhuysen, B. M. Nat. Nanotechnol. 2012, 7, 583-586.

[85] Karageorgiev, P.; Orendi, H.; Stiller, B.; Brehmer, L. Appl. Phys. Lett. 2001, 79, 1730-1732.

[86] Liu, Z.; Zhang, Y.; Kok, S. W.; Ng, B. P.; Soh, Y. C. Opt. Express 2010, 18, 3298-3310.

[87] Liu, Z.; Zhang, Y.; Kok, S. W.; Ng, B. P.; Soh, Y. C. Ultramicroscopy 2013 , 124, 26-34.

[88] Tranchida, D.; Diaz, J.; Schön, P.; Schönherr, H.; Vancso, G. J. Nanoscale 2011, 3, 233-239. 


\section{Chapter 3}

\section{Imaging ellipsometry of redox-responsive polymer films}

This chapter describes a novel approach to quantitatively visualize sub-nanometer height changes occurring in thin films of redox-active polymers upon reversible electrochemical oxidation/reduction in-situ and in real-time with electrochemical imaging ellipsometry (EC-IE). This approach is based on the utilization of a micro patterned substrate containing circular patterns of passive (non-redox active) 11mercapto-1-undecanol (MCU) within a redox-responsive oligoethylene sulfide endfunctionalized poly(ferrocenyldimethylsilane) (ES-PFS) film on a gold substrate. The non-redox responsive MCU layer was used as a molecular reference layer for the direct visualization of the thickness variations of the ES-PFS layer. The ellipsometric microscopy images were recorded in aqueous electrolyte solutions at potentials of $-0.1 \mathrm{~V}$ and $0.6 \mathrm{~V}$ vs. $\mathrm{Ag} / \mathrm{AgCl}$, corresponding to the reduced and oxidized redox states of ES-PFS, respectively. The ellipsometric contrast images showed a $37 \%( \pm 2)$ intensity increase in the ES-PFS layer upon oxidation. The thickness of the ES-PFS layer reversibly changed between $4.0 \mathrm{~nm}( \pm 0.1)$ and $3.4 \mathrm{~nm}( \pm 0.1)$ upon oxidation and reduction, respectively, as determined by IE. Additionally, electrochemical atomic force microscopy (EC-AFM) was used to verify the redox controlled thickness. The proposed method opens novel avenues to optically visualize rapid height changes occurring in redox-active polymer films in a fast and non-invasive manner. 
The contribution of this chapter is published in:

Cumurcu, A., Feng, X., Dos Ramos, L., Hempenius, M. A., Schön, P. M. and Vancso, G. J., Sub-nanometer expansions of redox responsive polymer films monitored by imaging ellipsometry, Nanoscale, 2014, DOI: $10.1039 / \mathrm{C} 4 \mathrm{NR} 02852 \mathrm{~J}$. 


\subsection{Introduction}

Growing demand for organic thin films in functional coatings, lithography resists, and other areas in nanotechnology make it inevitable to study dynamic processes such as film swelling and growth, or molecular adsorption in real time at the nanoscale [1-9]. For instance, in situ ellipsometry has been used to determine polymerization kinetics and polymer growth as well as morphological changes of polymer films upon environmental changes such as variations in solvent, temperature and $\mathrm{pH}$ in real time [1-8]. The thickness changes upon oxidation and reduction for redox-active monolayers were also determined by in situ ellipsometry $[10,11]$. However, conventional ellipsometry lacks the possibility of direct optical visualization of morphology variations of thin films.

Imaging ellipsometry (IE), a hybrid of ellipsometry and optical microscopy, combines the sub-nanometer vertical resolution of ellipsometry with the lateral resolution of optical microscopy [12]. The morphological structures of surfaces are imaged by using an objective and a CCD detector $[12,13]$. IE is based on the use of combined null and off-null ellipsometry. This means that while the optical signal on one area of the sample surface is minimized by null-ellipsometry conditions (areas appearing dark in the image), the un-nulled areas, simultaneously, provide a stronger signal (which appear bright in the image) [12]. IE is a label free and non-contact technique with in situ measurement possibilities, therefore it is wellsuited to monitor real time changes at surfaces of thin films [14-20]. For instance, using in situ IE, Schmaljohann et al. investigated the thermoresponsive aggregation behavior of a micropatterned hydrogel film in the region of $35-37^{\circ} \mathrm{C}[16]$. The temperature dependent swelling and collapse of the hydrogel film in water was monitored by recording the ellipsometric angle, $\Delta$, as a function of temperature. In another study by Faiss et al., the thickness variations of microstructured lipid bilayers upon temperature change were monitored by IE [17]. Ellipsometric thickness maps of 1,2-dimyristoyl-sn-glycero-3-phosphocholine (DMPC) bilayers showed a reversible thickness change between 13 and $46{ }^{\circ} \mathrm{C}$.

IE was also combined with other techniques such as electrochemical methods and microbeam grazing incidence small angle X-ray scattering ( $\mu$ GISAXS) $[15,18-20]$. For instance, in a study by Svoboda et al., in situ IE was combined with cyclic voltammetric deposition to monitor the film growth of conductive biocatalytic polymerized methylene green (poly-MG) films on platinum surfaces [15]. It was shown that the film growth was homogenous and resulted in an evenly distributed polymer film. Another hybrid IE technique was introduced by Körstgens et al. [18]. In this new tool a microbeam grazing incidence small angle X-ray scattering ( $\mu$ GISAXS) was coupled to an IE for structural and morphological characterization of nanostructures. To illustrate the capabilities of this tool, kinetics of the self-assembly of colloidal polymer nanospheres on a rough substrate were studied [18]. In another study by Yu et al., IE was combined with electrochemical methods to study electrostaticially driven adsorption of proteins on solid surfaces in real time [19]. The adsorption of fibrinogen on potential-controlled and non- 
controlled surfaces was measured in real time. Liu et al. used total internal reflection imaging ellipsometry (TIRIE) to monitor protein interaction processes in real time [20].

Macromolecules which contain organometallic groups or inorganic elements in their main chain are of particular interest in current research, since they not only possess the processability of polymers but also highly interesting optical, electrical, magnetic and chemical characteristics of the inorganic groups. [2123]. Poly(ferrocenylsilanes) (PFSs), containing alternating ferrocene and silicon units in their main chain, belong to this group of materials (Fig. 3.1) [24-26]. $\mathrm{PFS}$ is a redox active polymer which can be partially or fully oxidized using chemical or electrochemical procedures [27-30]. Therefore, PFS found use in various applications including ion recognition, biosensors, electrochemical sensing and molecular release [31-33]. Additionally, PFS was used as resist in different lithography techniques, such as UV photolithography, electron-beam (e-beam) lithography, as well as in more traditional lithography processes as an etch mask. The resulting metal-rich masking nano-structures offer etch resistance, catalytic, optical, sensing, electrical and magnetic properties [34-38].

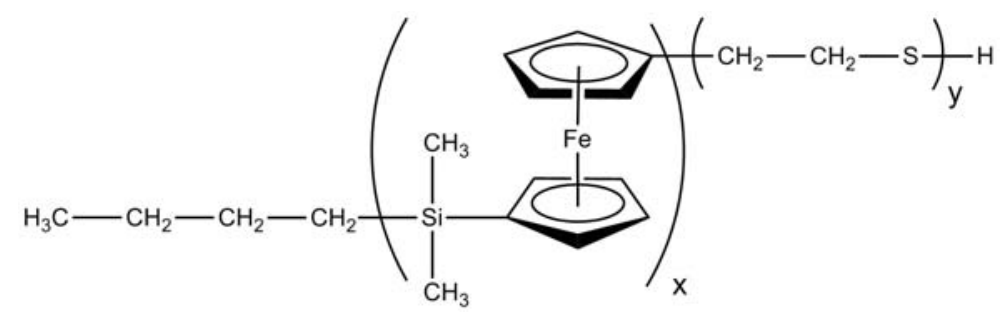

Figure 3.1: The structure of an oligoethylene sulfide end-functionalized poly(ferrocenyldimethylsilane) (ES-PFS).

The electrochemically induced morphology and volume changes of surface grafted PFS layers and also other redox-active polymers, such as polypyrrole (PPy) and polyaniline (PANI), were previously studied by spectroscopic ellipsometry and surface plasmon resonance spectroscopy under electrochemical control [10, 39, 40]. Electrochemical atomic force microscopy (EC-AFM) and microscopic observation simultaneous with voltammetric experiments were the only imaging techniques used to visualize the associated morphology changes [10, 39-42]. However, to the best of our knowledge, there are no reports in the literature on the imaging of thickness changes relative to a fixed layer with a fast, non-distractive method such as IE. For instance, sample areas of $600 \times 400 \mu \mathrm{m}^{2}$ can be imaged within two seconds with IE.

Soft lithography techniques including microcontact printing $(\mu \mathrm{CP})$, replica molding (REM), microtransfer molding ( $\mu \mathrm{TM})$, micromolding in capillaries (MIMIC), solvent assisted micromolding (SAMIM) etc., are cost-effective and fast microfabrication methods [43-50]. Soft lithography techniques allow one to control the chemistry of the patterned surfaces and to manufacture patterned surfaces with complex organic groups found in biology and biochemistry [51]. Patterned elas- 
tomers, such as polyurethanes, polyimides, poly(dimethylsiloxanes) (PDMS), are used as stamps in all soft lithography techniques [52]. The pattern on elastomeric stamps such as PDMS is produced by molding the PDMS on a mask which is fabricated by photolithography. Before casting the PDMS, the master must be functionalized with a silane vapor, such as a fluorosilane, to cap the reactive -OH groups with inert $-\mathrm{CH}_{3}$ or $-\mathrm{CF}_{3}$ groups so that the elastomer does not adhere to the master [53].

In $\mu \mathrm{CP}$, an ink solution (containing e.g. thiols or silanes) is used to coat the patterned elastomer (generally PDMS) and the inked stamp is then put in conformal contact with the surface [43]. Consequently, a monolayer of the ink solution is formed in the areas of contact between the substrate and the PDMS and the areas with no contact remain bare. PDMS stamps can be used hundreds of times without degradation. They are durable and inert to most of the ink solutions and chemically resistant to many solvents. The elastomeric properties of PDMS allow stamps of PDMS to achieve conformal contact with the substrate with little or no applied pressure. However, its high compressibility may cause PDMS to deform and collapse resulting in defects in the pattern [54]. Microstructures of self-assembled monolayers (SAMs) can be prepared by $\mu \mathrm{CP}$ in seconds without the necessity of expensive clean room facilities [55].

In this chapter, the redox-induced morphology and volume changes of an oligoethylene sulfide end-functionalized poly (ferrocenyldimethylsilane) (ES-PFS) film on a gold substrate was visualized versus a 11-mercapto-1-undecanol (MCU) layer in situ by electrochemical imaging ellipsometry (EC-IE). A micro-patterned film of the MCU layer (100 $\mu \mathrm{m}$ diameter circles) was prepared by $\mu \mathrm{CP}$ and subsequently the unpatterned areas were filled with the ES-PFS layer. The MCU film served as zero-level, inert reference layer to monitor sub nm thickness variations of the ES-PFS film. The thickness changes were verified in situ by IE and AFM under electrochemical control. This strategy allows one to precisely and accurately measure thickness changes, and simultaneously observe at film surfaces morphology variations. This approach may find applications in studies of thin film erosion, photoresist pattern development, use of responsive layers in (bio)sensing and actuation, in fluidics, and other nanotechnologically relevant areas.

\subsection{Results and discussion}

\subsubsection{Micro-patterned ES-PFS/MCU film preparation}

The redox-induced morphology and volume changes of a ES-PFS film on gold were monitored in situ by EC-IE. For this purpose, a micro-patterned ES-PFS/MCU sample was prepared on a gold substrate by $\mu \mathrm{CP}$. The sample preparation steps are shown in Fig. 3.2. Firstly, the MCU layer was printed on a gold substrate using a PDMS stamp (Fig. 3.2, step 1 to 4). Next, the un-patterned areas on the 
gold substrate were filled with the ES-PFS layer by pouring the ES-PFS solution on the sample (Fig. 3.2, step 5). Subsequently, the sample was washed with large amounts of THF and ethanol to remove any traces of physisorbed ES-PFS and MCU layers (Fig. 3.2, step 6). Finally, the ES-PFS/MCU sample contained circle patterns of non-redox active MCU with a surrounding redox active ES-PFS layer (Fig. 3.2, step 7).

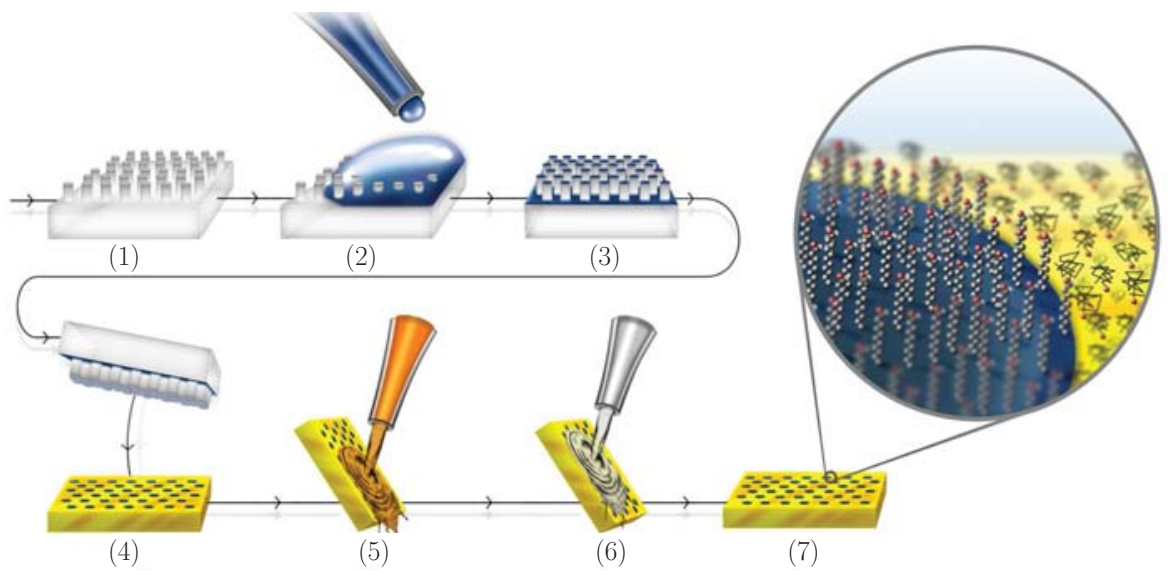

Figure 3.2: Preparation of the micro-patterned ES-PFS/MCU sample by $\mu \mathrm{CP}$ : (1) PDMS elastomeric stamp with pillars, (2) the stamp was covered by the ink solution of MCU, (3) the stamp was dried until no visible ink remains, (4) the ink was printed by contacting the inked stamp with a gold substrate, (5) ES-PFS solution was poured onto the MCU patterned gold substrate, (6) the substrate was washed with THF and ethanol, (7) the final micro-patterned ES-PFS/MCU sample. The inset in the step 7 shows a zoomed scheme of the patterned MCU monolayer (represented with a blue background) and the ES-PFS layer present on the outside of the patterned area (represented with a yellow background).

As expected, the sub-nanometer thickness variations in ES-PFS films, it is important to define an inert zero level as reference. Thus it is of pivotal importance to exclude possible nonspecific adsorption of PFS to the MCU patterns. In order to determine the adsorption of ES-PFS on the MCU layer, a non-patterned sample was prepared first by immersing the gold substrate into the MCU solution overnight to form the thiol monolayer on the entire gold surface. The same amount of ES-PFS solution was poured on this sample as in the micro-patterned ES-PFS/MCU sample preparation and the sample was then washed with the same amount of THF and ethanol. The XPS spectra of this non-patterned sample did not show any traces of Fe, proving the absence of ES-PFS on the MCU pattern after this fabrication procedure (Fig. 3.3). 


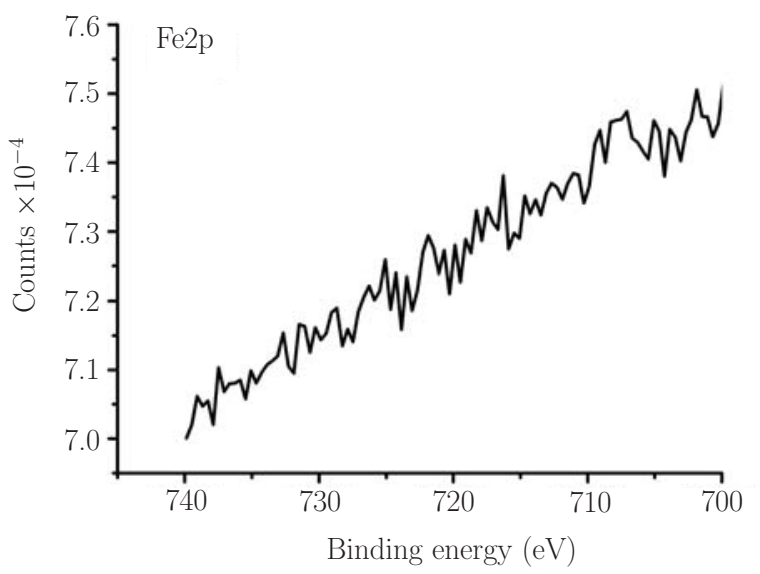

Figure 3.3: XPS element spectra scan of Fe in characterization sample of MCU. See the text for the details.

\subsubsection{Electrochemistry of the ES-PFS/MCU sample}

A cyclic voltammogram (CV) of the micro-patterned ES-PFS/MCU sample on a gold substrate, recorded with the EC-IE, is shown in Fig. 3.4. The double-wave peaks in the $\mathrm{CV}$ are indicative for PFS and reflect the intermetallic coupling between neighboring iron centers in the polymer chain $[10,28,30]$. In the first oxidation wave, ferrocene units in alternating positions are oxidized, generating positively charged ferrocenium $(\mathrm{Fc}+)$ moieties, followed by oxidation of the remaining ferrocenes in the second wave at higher potential $[56,57]$. Broadening of the second wave is attributed to intermetallic interactions with a variable number of oxidized neighboring units [10].

Additionally, the surface coverage of the ferrocene sites $\left(\Gamma_{F c}\right)$ was calculated as $1.76 \times 10^{-9} \mathrm{~mol} \mathrm{~cm}^{-2}$ using the following equation:

$$
\Gamma_{F c}=\frac{Q_{F c}}{n F A}
$$

where $n$ is the number of electrons involved in the electron transfer (here $n_{e}=1$ ), $F$ is the Faraday constant $\left(F=96485 \mathrm{C} \mathrm{mol}^{-1}\right), A$ is the geometric surface area of the electrode $\left(A=1 \mathrm{~cm}^{2}\right)$. $\mathrm{Q}_{F c}$ is the charge passed for the oxidation/reduction of ferrocene sites and was determined by integrating the areas under the redox peaks. This corresponds to the number of grafted chains per unit area of 0.17 chain nm $^{-2}$. The surface coverage was calculated using a CV of a full ES-PFS layer prepared on a gold substrate by the same procedure of the ES-PFS filling of the micropatterned ES-PFS/MCU sample (Fig. 3.5). This full ES-PFS layer was used to 


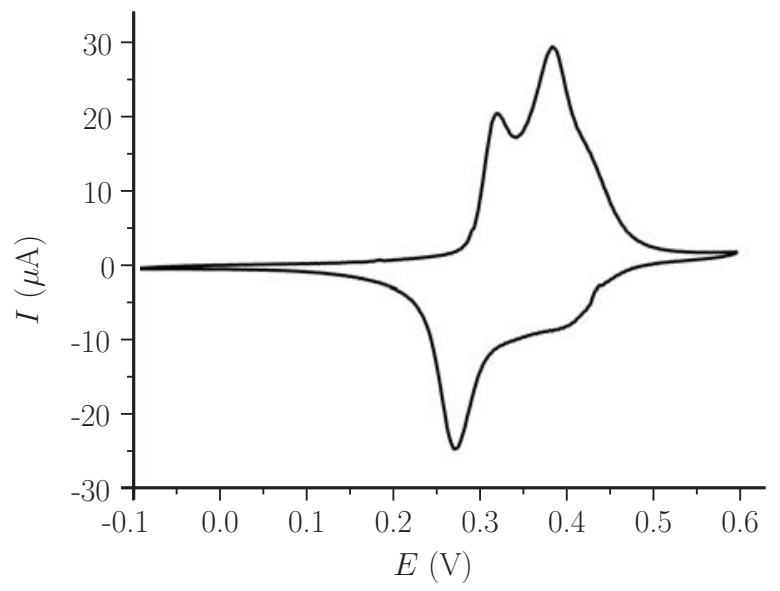

Figure 3.4: Cyclic voltammogram recorded for a patterned PFS/MCU monolayer on a gold substrate at a scan rate of $50 \mathrm{mV} \mathrm{s}^{-1}$ in $0.1 \mathrm{M} \mathrm{NaClO}_{4}$ with a $\mathrm{Ag} / \mathrm{AgCl}$ reference electrode, and a $\mathrm{Pt}$ counter electrode.

determine the surface area of the ES-PFS accurately. A previous study on ES-PFS layers reported a surface coverage of $2.3 \times 10^{-9} \mathrm{~mol} \mathrm{~cm}{ }^{-2}$ for the ES-PFS with 50 repeating units (note that ES-PFS with 60 repeating units was used) [28]. The difference in the surface coverage is due to the significantly shorter immersion time of the gold substrate in the ES-PFS solution in our procedure. A short immersion time reduces physisorption of the ES-PFS on the MCU layer.

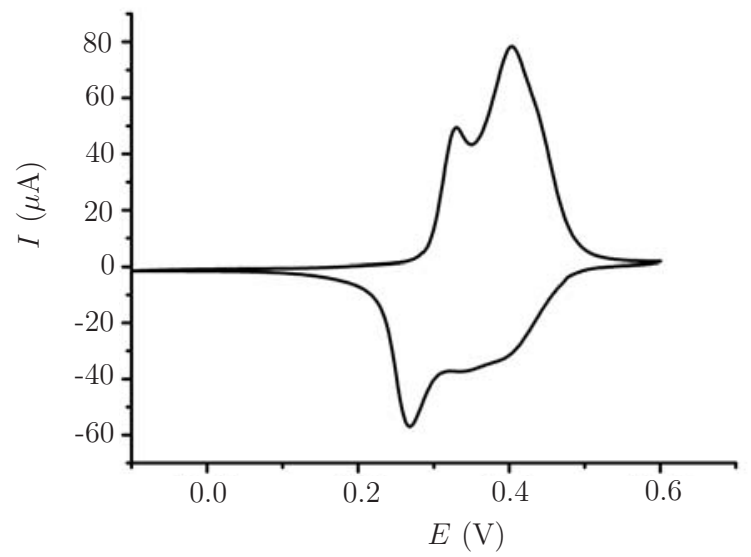

Figure 3.5: Cyclic voltammogram of an ES-PFS layer on gold captured at $50 \mathrm{mV} \mathrm{s}^{-1}$ scan rate. The reference electrode is $\mathrm{Ag} / \mathrm{AgCl}$, the counter electrode is $\mathrm{Pt}$ and $0.1 \mathrm{M} \mathrm{NaClO}_{4}$ is used as an electrolyte. 


\subsubsection{Thickness measurements by EC-IE}

The thickness changes induced upon electrochemical oxidation and reduction were monitored in situ by EC-IE. Fig. 3.6 shows the graphs of ellipsometric angles $(\Delta$ and $\Psi$ ) and the film thicknesses measured at oxidized and reduced states of the ES-PFS layer, respectively. The measurements for the oxidized and reduced states were recorded after keeping the potential at 0.6 and $-0.1 \mathrm{~V}$ for $2 \mathrm{~min}$. In cycle 1 (Fig. 3.6), the ES-PFS layer was in the oxidized state revealing a film thickness of $4.1 \mathrm{~nm}$ corresponding to $\Delta=120.814^{\circ}$ and $\Psi=44.063^{\circ}$. The film was then electrochemically reduced by keeping the potential at $-0.1 \mathrm{~V}$ for $2 \mathrm{~min}$ and showed $\Delta=121.206^{\circ}$ and $\Psi=44.147^{\circ}$ values corresponding to a film thickness of $3.4 \mathrm{~nm}$ (depicted as cycle 2 in Fig. 3.6). Repetitive cycling proved reversibility of the thickness changes upon oxidation and reduction. The average thickness increase was found to be $20 \%$ upon oxidation over three selected region of interests (ROIs) of ES-PFS. The thickness increase of the PFS layer upon oxidation was due to the repulsive interactions between $\mathrm{Fc}^{+}$centers in the polymer and chain stiffening of the oxidized backbone $[10,58]$.

In a previous study thickness changes of ES-PFS layers were determined upon oxidation via surface plasmon resonance spectroscopy (SPR) and spectroscopic ellipsometry [10]. SPR measurements, using a He/Ne laser with a wavelength of $632.8 \mathrm{~nm}$, revealed that the thickness increase from the neutral state to the fully oxidized state was $19 \%$ for a ES-PFS layer chains with degree of polymerization $D P_{n}=50$. Spectroscopic ellipsometry measurements, performed in the visible region between 310 and $820 \mathrm{~nm}$, showed that the thickness change of the same layer upon oxidation was $14 \%$ [10].

As seen in Fig. 3.6 ellipsometric angles decrease upon oxidation and increase upon reduction. The change in $\Delta\left(\sim 0.38^{\circ}\right)$ upon oxidation and reduction is much larger than the change in $\left(\sim 0.074^{\circ}\right)$. This is expected since for thin films $\Psi$ is much less sensitive to thickness changes compared to $\Delta[59]$. It is also important to know that the IE instrument standard deviations of $\Delta$ and $\Psi$ are approximately $0.004^{\circ}$. This value was determined from the ES-PFS layer on a gold substrate in the aqueous $\mathrm{NaClO}_{4}$ solution by recording the ellipsometric angles on the same area. The standard deviations of the ellipsometric angles were calculated from 22 data points and averaged over the values determined from three different areas. Additionally, the MCU layer thickness was found to be $1.6 \mathrm{~nm}( \pm 0.2)$. The thickness of the MCU layer did not change upon oxidation and reduction, since it does not contain any redox active units. 
(a)

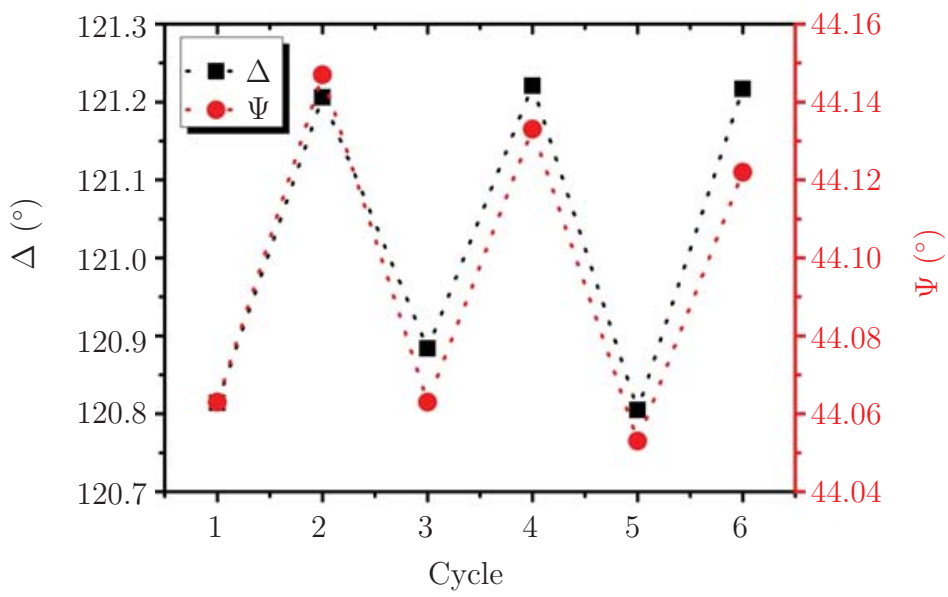

(b)

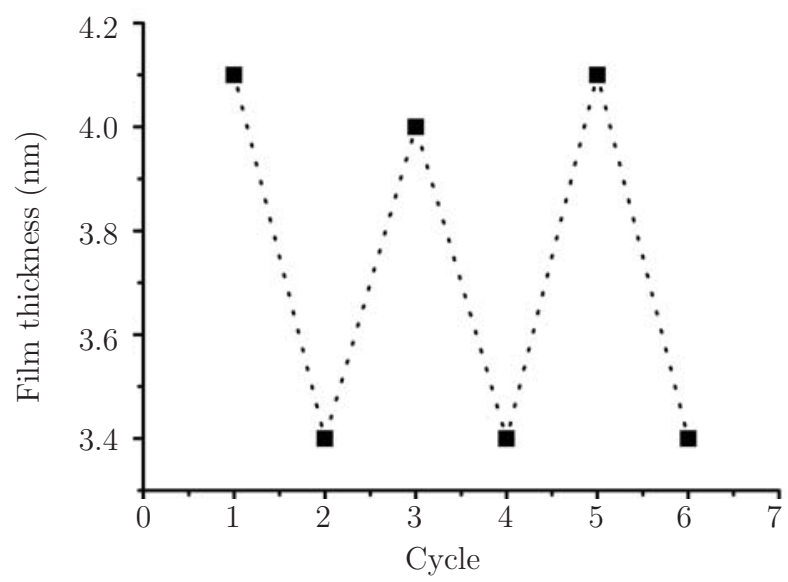

Figure 3.6: Ellipsometric data and film thickness of the ES-PFS layer: In (a), $\Delta$ (left) and $\Psi$ (right) variations and in (b) the film thicknesses are plotted versus oxidation and reduction cycles. The first cycle represents the oxidized state. The dotted lines are included for guidance.

\subsubsection{In situ ellipsometric contrast images}

EC-IE was used to visualize the redox induced thickness change of the ES-PFS layer. Fig. 3.7 shows the ellipsometric contrast images of the ES-PFS/MCU sample collected in situ with EC-IE and the average intensity changes for the ES-PFS and the MCU layers. The ellipsometric contrast image represents the reflected light intensity detected by the CCD camera at the optimized position of the polarizing components to obtain maximal contrast and lateral resolution of the pattern. 

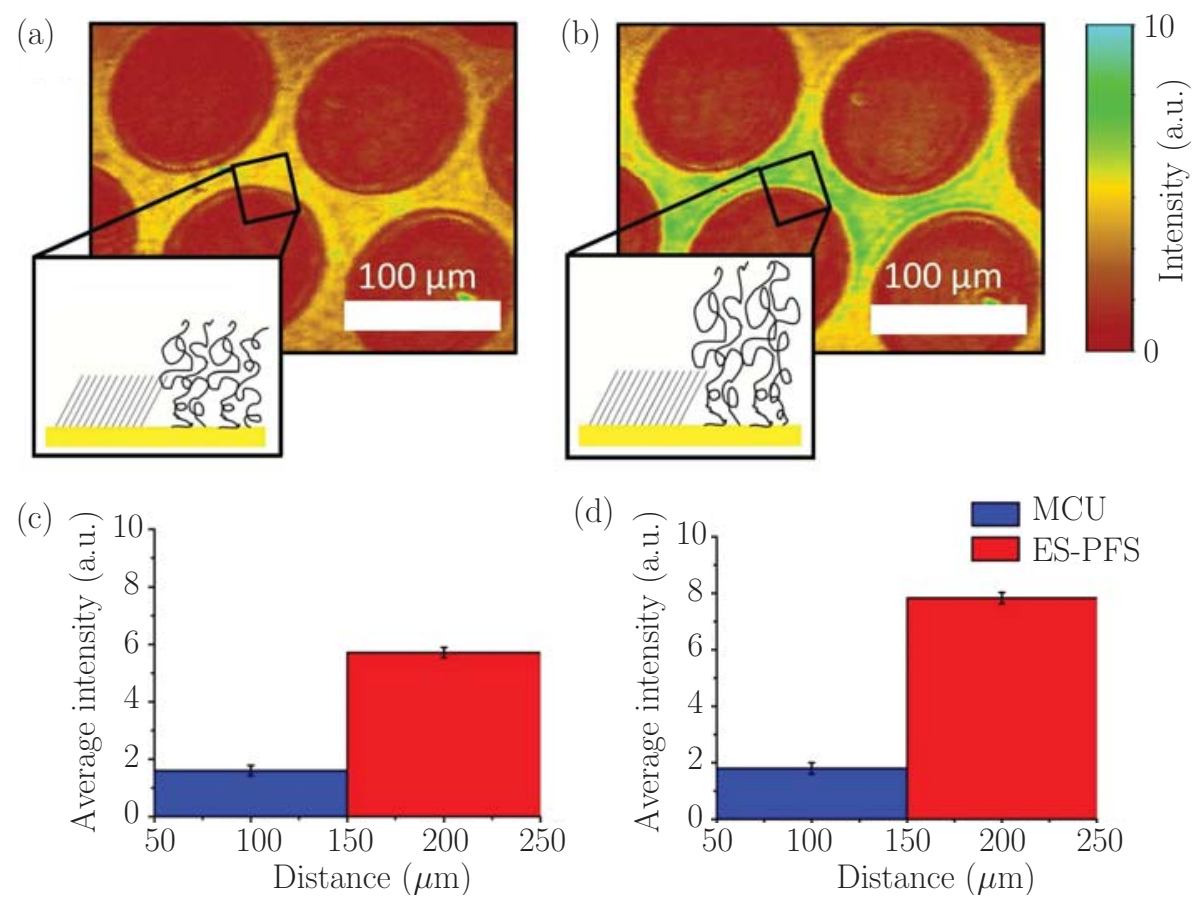

Figure 3.7: Ellipsometric contrast images of the micro patterned ES-PFS/MCU sample captured at potentials (a) -0.1 and (b) $0.6 \mathrm{~V}$ vs Ag/ $\mathrm{AgCl}$. The insets in (a) and (b) show the schemes of the thickness changes of the ES-PFS layer against the stationary MCU layer. The graphs in (c) and (d) show the average intensities of the MCU (blue column) and ES-PFS (red column) layers determined from the images captured at the potentials -0.1 and $0.6 \mathrm{~V}$, respectively. The error bars show the standard deviation of the average intensities.

With the nulling mode, the signal on one area of the sample surface is minimized while the other un-nulled areas provide a strong signal simultaneously. The images in Fig. 3.7(a) and (b) show the real time intensity at the reduced and the oxidized states of the sample, respectively. The MCU layer (the circular areas) and the ESPFS layer (surrounding areas) revealed different intensities due to the thickness and refractive index differences of the layers. However, more importantly the images showed a clear intensity increase for the ES-PFS regions upon oxidation, while the MCU regions maintained the same intensity. This result is attributed to the change in the optical thickness of the ES-PFS layer upon oxidation due to conformational changes in the polymer chains by the increase in the charge density.

The intensity in the central part of the ellipsometric contrast images is higher than in the margins due to the focus of the laser beam. However, the intensity change of the ES-PFS layer between the reduced and oxidized state is the same for all the areas of the image. 
The average intensities of the ES-PFS and MCU layers were determined over five cross-sections of $100 \mu \mathrm{m}$ distances by averaging 450 data points in each crosssection. The intensity of the ES-PFS layer was increased $37 \%( \pm 2)$ upon oxidation as opposed to the stationary MCU layer. IE, as a relative approach, provides higher sensitivity since it does not require data fitting to simulations and assumptions such as the refractive index of the polymer film, the medium and the material density uniformity. Additionally, since IE is a fast, non-distractive method and has high precision, it is a better suited technique for determining the changes in the dynamic processes compared to other imaging techniques such as AFM, for instance.

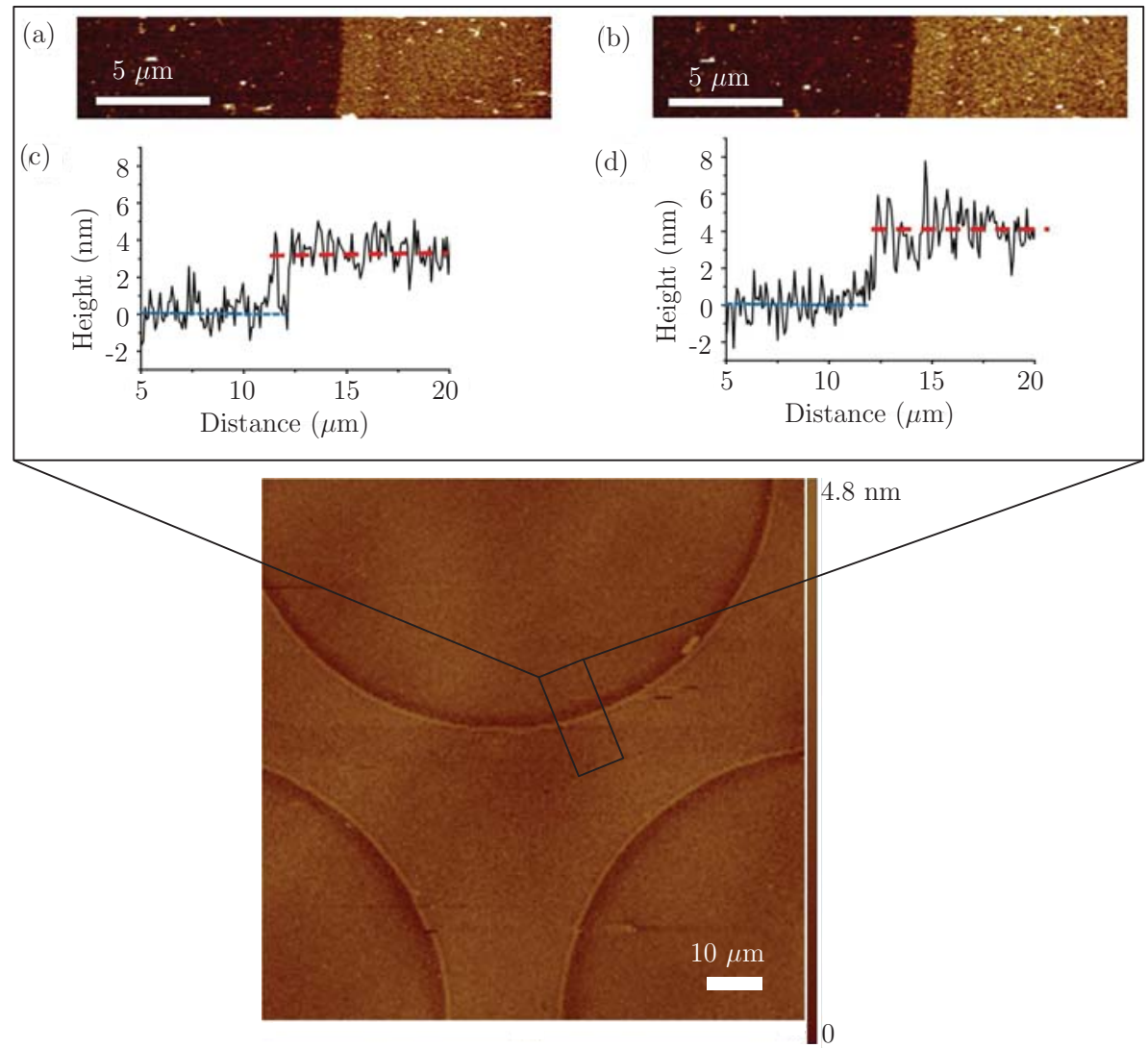

Figure 3.8: EC-AFM topography images of the micro patterned ES-PFS/MCU sample: the large scale image at the bottom shows the AFM image of the micropatterned structure of the sample. The inset (top images) shows a zoomed area indicated by the frame shown in the bottom image. The step height images and the corresponding cross sections which was calculated by the average height of (a) reduced and (b) oxidized states of the sample show the response of the redox-active PFS layer. 
The real time ellipsometric microscopy images were recorded with EC-IE, continuously while cycling the potential between -0.1 and $0.6 \mathrm{~V}$ in a video recording. This recording is provided in the http://www.mtpgroup.nl/diss-aysegul-cumurcu.aspx website. The video shows the reversibility of the thickness change simultaneously with the potential change. In addition, the movie provides further evidence of the two step oxidation, as can be seen from a corresponding stepwise intensity increase and decrease upon oxidation and reduction, respectively.

\subsubsection{AFM topography imaging}

EC-AFM was used to verify the thickness changes of the ES-PFS layer against the stationary MCU layer in situ as a function of the applied potential (Fig. 3.8). The topography of the micro-patterned ES-PFS/MCU sample was imaged at potentials -0.2 and $0.6 \mathrm{~V}$. The ES-PFS layer revealed a rougher surface at the oxidized state compared to the reduced state [10]. The height difference of the ES-PFS layer from reduced to oxidized state was $0.6 \mathrm{~nm}$ which corresponded to a height change of $18 \%$ against the stationary MCU layer. Here peak force tapping AFM was applied which utilizes the maximum loading force as feedback and thus operates in the repulsive contact regime. In this manner, possible influences on the measured height profiles due to electrostatic interactions changes are eliminated.

\subsection{Conclusions}

In this chapter, a novel concept was proposed to quantitatively visualize subnanometer height changes occurring in thin films of redox active polymers upon reversible electrochemical oxidation/reduction in-situ and in real time combined electrochemistry imaging ellipsometry (EC-IE). This approach is based on the use of micropatterned thin films, including patterns of inert (non-redox active) alkanethiols which function as zero level molecular reference. As the reference layer remains unperturbed upon electrode potential changes, it maintains a constant height during the experiment. This method allows one to monitor the two step oxidation of surface grafted PFS on patterned areas of $600 \times 400 \mu \mathrm{m}^{2}$ within two seconds. From imaging ellipsometry data, a height change of $6 \pm 1 \AA$ can be deduced, which was also confirmed from electrochemical AFM measurements. The proposed method opens entirely novel avenues to optically visualize minute and rapid changes occurring in redox-active polymer films in a fast and non-invasive manner. 


\subsection{Experimental section}

Materials: MCU and 1H,2H,2H-perfluorodecyltrichlorosilane were purchased from Sigma-Aldrich. PDMS prepolymer (Sylgard silicone elastomer 184) and curing agent (Sylgard silicone elastomer 184 curing agent) were provided from Dow Corning Corporation. Tetrahydrofuran (THF) and ethanol were acquired from Biosolve (Valkenswaard, The Netherlands). Hydrogen peroxide $\left(\mathrm{H}_{2} \mathrm{O}_{2}\right) 30 \%$ was obtained from Merck (Darmstadt, Germany). Sulfuric acid $\left(\mathrm{H}_{2} \mathrm{SO}_{4}\right)$ 95-97\% was obtained from Sigma-Aldrich (St. Louis, MO, USA). All materials were used as received. Milli-Q water was produced by a Millipore Synergy system (Billereca, MA, USA). ES-PFS $\left(\mathrm{M}_{n}=22465 \mathrm{~g} / \mathrm{mol}, \mathrm{M}_{w}=26063 \mathrm{~g} / \mathrm{mol}, \frac{M_{w}}{M_{n}}=1.16\right)$ was prepared by treating living PFS with ethylene sulfide according to a previously published procedure [30].

Sample preparation: Gold substrates (silicon wafer, $10 \mathrm{~nm}$ chromium adhesive layer and $100 \mathrm{~nm}$ gold layer) were prepared using the Balzer BAK 600 evaporation system. Root mean square (RMS) roughness of a gold substrate was $0.89 \mathrm{~nm}$ for a $40 \times 40 \mu \mathrm{m}^{2}$ area as determined by AFM. Prior to use, the gold substrates with dimensions of $2.5 \times 1.5 \mathrm{~cm}^{2}$ were cleaned in piranha $\left(30 \% \mathrm{H}_{2} \mathrm{O}_{2} / 70 \% \mathrm{H}_{2} \mathrm{SO}_{4}\right)$ for $20 \mathrm{~s}$ and rinsed with Milli-Q water and ethanol. Then the substrates were dried in a stream of nitrogen. Caution: Piranha solution is highly corrosive. Extreme care should be taken when handling Piranha solution and only small quantities should be prepared. The elastomeric stamp was prepared by pouring a mixture of PDMS prepolymer and its curing agent (10:1 by weight) onto a silicon master with features of a hole array of $100 \mu \mathrm{m}$ circles at a depth of $600 \mathrm{~nm}$. Prior to use, the master was treated with an anti-adhesion layer of $1 \mathrm{H}, 1 \mathrm{H}, 2 \mathrm{H}, 2 \mathrm{H}-$ Perfluorodecyltrichlorosilane to facilitate remolding. The master had dimensions of $1.5 \times 1.5 \mathrm{~cm}^{2}$ with a patterned region of $1 \times 1 \mathrm{~cm}^{2}$. After curing at $70{ }^{\circ} \mathrm{C}$ for 12 hours, the PDMS was carefully peeled off the stamp at room temperature. The features of the master were transferred to the PDMS stamp as pillars of the same dimensions.

A $2 \mathrm{mM}$ solution of MCU in ethanol was used as ink for the $\mu \mathrm{C}$ printing. First, the ink solution was applied to the surface of the PDMS stamp by a cotton Q-tip and then dried with a stream of $\mathrm{N}_{2}$ for $\sim 1$ min [60, 61]. The dry PDMS stamp, meaning no visible ink was present on the surface, was brought into contact with the gold substrate for $30 \mathrm{~s}$. It is important to note that gentle tapping on the PDMS stamp may be needed to ensure that air bubbles are not trapped between the PDMS stamp and the gold substrate [62]. After removing the stamp, a $5 \mathrm{ml}$ solution of ES-PFS ( $1 \mathrm{mg} \mathrm{m}{ }^{-1}$ solution in THF) was poured on the substrate. Next, the substrate was washed with large amounts of THF and ethanol at room temperature. Finally, the substrate was dried in a stream of $\mathrm{N}_{2}$. 
Characterization techniques:

Electrochemical imaging ellipsometry (EC-IE)

In-situ IE measurements were performed with a Multiskop imaging ellipsometer (Optrel GBR, Kleinmachnow, Germany) under electrochemical potential control with an Autolab PGSTAT10 potentiostat (Ecochemie, Utrecht, The Netherlands) in a liquid cell with a volume of $0.7 \mathrm{ml}$ and PEEK cover and Teflon tubing (Nanofilm surface analysis, Halcyonics $\mathrm{GmbH}$ ). The angle of incidence was set to $60^{\circ}$, which assured that the light path was oriented normal to the window of the liquid cell [16]. The IE was operated in the Polarizer-Compensator-SampleAnalyzer (PCSA) configuration. The local height profile of the polymer film was obtained using a microscope objective (M-Plan APO 10×, Mitutoyo, Neuss, Germany) and a CCD camera with a resolution of $640 \times 480$ pixels. The sample was illuminated with a He-Ne laser $(\lambda=632.8 \mathrm{~nm})$.

The electrochemical experiments (cyclic voltammetry) were done in a liquid cell with a three-electrode configuration. The gold substrate with the polymer coating was used as working electrode, in combination with $\mathrm{Ag} / \mathrm{AgCl}$ reference and $\mathrm{Pt}$ counter electrode. Measurements were done in $0.1 \mathrm{M} \mathrm{NaClO}_{4}$ aqueous solution. The cyclic voltammograms were recorded between -0.1 and $0.6 \mathrm{~V}$ (versus $\mathrm{Ag} / \mathrm{AgCl}$ ) at $50 \mathrm{mV} \mathrm{s}^{-1}$ scan rate. The IE images were captured after keeping the sample at potentials of -0.1 and $0.6 \mathrm{~V}$ for two minutes each, to insure the complete redox-response of the whole polymer layer. The images were corrected for the distortion caused by the oblique angle of incidence [12]. The average intensities were calculated from five cross-sections over a $100 \mu \mathrm{m}$ distance with 450 data points on each cross-section for the both layers.

Thicknesses of the ES-PFS layers were determined by IE from the ROIs containing the ES-PFS layers on the sample surface. A commercial software supplied with the Multiskop setup was used to fit a three-layer model (gold/ES-PFS/water) using Fresnel theory [63]. The refractive index of the ES-PFS layer was taken as $n=1.687$ for both the oxidized and the reduced state [10]. Refractive index and the extinction coefficient of the gold substrate were determined experimentally by ellipsometry as 0.175 and 3.519 , respectively.

Electrochemical atomic force microscopy (EC-AFM)

AFM measurements were performed in an EC-AFM setup using a Multimode 8 AFM (Bruker Nano Surfaces, Santa Barbara, CA, USA) with a NanoScope V controller and an electrochemical liquid cell in combination with an Autolab PGSTAT10 potentiostat (Ecochemie, Utrecht, The Netherlands). Commercially available V-shaped silicon nitride cantilevers (DNP, Bruker AFM probes, Camarillo, CA, USA) with nominal spring constants of $0.35 \mathrm{~N} \mathrm{~m}^{-1}$ were used for imaging. Peak Force Tapping Mode AFM was done at a constant oscillation of the sample at $2 \mathrm{kHz}$ using amplitudes of $30-120 \mathrm{~nm}$ and peak forces of $100 \mathrm{pN}$. Scanning was performed at a speed of $0.977 \mathrm{~Hz}$. 
The EC-AFM experiments were performed in a closed electrochemical liquid cell (DI) with approximately $50 \mu \mathrm{l}$ inner volume. The liquid cell contained a threeelectrode system including the polymer covered gold substrate as working, a $\mathrm{Pt}$ wire as counter and $\mathrm{Ag}$ wire as reference electrode. A $0.1 \mathrm{M} \mathrm{NaClO}_{4}$ aqueous solution was used as electrolyte. The potential was cycled between -0.2 and $0.6 \mathrm{~V}$ at a scan rate of $50 \mathrm{mV} \mathrm{s}^{-1}$ and the AFM height images were captured after keeping the sample two minutes at the oxidized or reduced state, respectively. Before and after AFM imaging, cyclic voltammograms were recorded, to determine the surface concentration of the PFS and to verify proper electrochemistry of the modified substrate.

It should be noted that the EC-IE and EC-AFM measurements were done with different set-ups and electrochemical liquid cells. While for the EC-AFM measurements a silver wire was used as reference electrode, for the EC-IE measurements an $\mathrm{Ag} / \mathrm{AgCl}$ electrode was used. Therefore, the respective minimum and maximum vertex potentials were chosen according to the specific cyclic voltammogram recorded for each experiment, proofing complete oxidation or reduction, respectively. Accordingly, a slightly more positive potential was chosen for the EC-AFM $(-0.1 \mathrm{~V})$ compared to the EC-IE experiments $(-0.2 \mathrm{~V})$.

Image processing and data analysis were performed with the NanoScope software version 8.15, and NanoScope Analysis software version 1.4. To quantify the average height variation, step analysis was performed which makes relative height measurements between two regions (steps) on sample surfaces.

\section{$X$-ray photoelectron spectroscopy (XPS)}

The XPS spectra was recorded on a PHI Quantera XPS microprobe (Physical Electronics Inc., Chanhassen, MN, USA) using a monochromatic X-ray beam (Al $\mathrm{K} \alpha$ monochromatic at $1486.6 \mathrm{eV}, 200 \mu \mathrm{m}$ diameter, $50 \mathrm{~W}$ ) at a take-off angle of $45^{\circ}$ immediately after sample preparation. 


\section{Bibliography}

[1] Halthur, T. J.; Claesson, P. M.; Elofsson, U. M. J. Am. Chem. Soc. 2004, 126, 17009-17015.

[2] Harris, J. J.; Bruening, M. L. Langmuir 2000, 16, 2006-2013.

[3] Campoy-Quiles, M.; Ferenczi, T.; Agostinelli, T.; Etchegoin, P. G.; Kim, Y.; Anthopoulos, T. D.; Stavrinou, P. N.; Bradley, D. D. C.; Nelson, J. Nat. Mater. 2008, 7, 158-164.

[4] Papanu, J. S.; Hess, D. W.; Bell, A. T.; Soane, D. S. J. Electrochem. Soc. 1989, 136, 1195-1200.

[5] Santonicola, M. G.; de Groot, G. W.; Memesa, M.; Meszynska, A.; Vancso, G. J. Langmuir 2010, 26, 17513-17519.

[6] Sui, X. F.; Zapotoczny, S.; Benetti, E. M.; Memesa, M.; Hempenius, M. A.; Vancso, G. J. Polym. Chem-Uk 2011, 2, 879-884.

[7] Minko, S.; Sidorenko, A.; Stamm, M.; Gafijchuk, G.; Senkovsky, V.; Voronov, S. Macromolecules 1999, 32, 4532-4538.

[8] Halthur, T. J.; Elofsson, U. M. Langmuir 2004, 20, 1739-1745.

[9] Pasche, S.; De Paul, S. M.; Vörös, J.; Spencer, N. D.; Textor, M. Langmuir 2003, 19, 9216-9225.

[10] Peter, M.; Hempenius, M. A.; Kooij, E. S.; Jenkins, T. A.; Roser, S. J.; Knoll, W.; Vancso, G. J. Langmuir 2004, 20, 891-897.

[11] Carlin, C. M.; Kepley, L. J.; Bard, A. J. J. Electrochem. Soc. 1985, 132, $353-359$.

[12] Asinovski, L.; Beaglehole, D.; Clarkson, M. T. Phys. Status Solidi A 2008, 205, 764-771.

[13] Jin, G.; Jansson, R.; Arwin, H. Rev. Sci. Instrum. 1996, 67, 2930-2936.

[14] Munteanu, S.; Garraud, N.; Roger, J. P.; Amiot, F.; Shi, J.; Chen, Y.; Combellas, C.; Kanoufi, F. Anal. Chem. 2013, 85, 1965-1971.

[15] Svoboda, V.; Cooney, M. J.; Rippolz, C.; Liaw, B. Y. J. Electrochem. Soc. 2007, 154, D113-D116.

[16] Schmaljohann, D.; Nitschke, M.; Schulze, R.; Eing, A.; Werner, C.; Eichhorn, Y. J. Langmuir 2005, 21, 2317-2322.

[17] Faiss, S.; Schuy, S.; Weiskopf, D.; Steinem, C.; Janshoff, A. J. Phys. Chem. B 2007, 111, 13979-13986. 
[18] Körstgens, V.; Wiedersich, J.; Meier, R.; Perlich, J.; Roth, S. V.; Gehrke, R.; Müller-Buschbaum, P. Anal. Bioanal. Chem. 2010, 396, 139-149.

[19] Yu, Y.; Jin, G. J. Colloid Interf. Sci. 2005, 283, 477-481.

[20] Liu, L.; Chen, Y. Y.; Meng, Y. H.; Chen, S.; Jin, G. Thin Solid Films 2011, 519, 2758-2762.

[21] Nguyen, P.; Gomez-Elipe, P.; Manners, I. Chem. Rev. 1999, 99, 1515-1548.

[22] Whittell, G. R.; Manners, I. Adv. Mater. 2007, 19, 3439-3468.

[23] Trippe, G.; Ocafrain, M.; Besbes, M.; Monroche, V.; Lyskawa, J.; Le Derf, F.; Salle, M.; Becher, J.; Colonna, B.; Echegoyen, L. New. J. Chem. 2002, 26, 1320-1323.

[24] Manners, I. Macromol. Symp. 2003, 196, 57-62.

[25] Kulbaba, K.; Manners, I. Macromol. Rapid. Comm. 2001, 22, 711-724.

[26] Eloi, J. C.; Chabanne, L.; Whittell, G. R.; Manners, I. Mater. Today 2008, 11, 28-36.

[27] Ahmed, R.; Hsiao, M. S.; Matsuura, Y.; Houbenov, N.; Faul, C. F. J.; Manners, I. Soft Matter 2011, 7, 10462-10471.

[28] Peter, M.; Lammertink, R. G. H.; Hempenius, M. A.; Vancso, G. J. Langmuir 2005, 21, 5115-5123.

[29] Manners, I. Chem. Commun. 1999, 857-865.

[30] Peter, M.; Lammertink, R. G. H.; Hempenius, M. A.; van Os, M.; Beulen, M. W. J.; Reinhoudt, D. N.; Knoll, W.; Vancso, G. J. Chem. Commun. 1999, 359-360.

[31] Puzzo, D. P.; Arsenault, A. C.; Manners, I.; Ozin, G. A. Angew. Chem. Int. Edit. 2009, 48, 943-947.

[32] Feng, X. L.; Cumurcu, A.; Sui, X. F.; Song, J.; Hempenius, M. A.; Vancso, G. J. Langmuir 2013, 29, 7257-7265.

[33] Sui, X. F.; Feng, X. L.; Song, J.; Hempenius, M. A.; Vancso, G. J. J. Mater. Chem. 2012, 22, 11261-11267.

[34] Cheng, A. Y.; Clendenning, S. B.; Yang, G. C.; Lu, Z. H.; Yip, C. M.; Manners, I. Chem. Commun. 2004, 780-781.

[35] Clendenning, S. B.; Aouba, S.; Rayat, M. S.; Grozea, D.; Sorge, J. B.; Brodersen, P. M.; Sodhi, R. N. S.; Lu, Z. H.; Yip, C. M.; Freeman, M. R.; Ruda, H. E.; Manners, I. Adv. Mater. 2004, 16, 215-219.

[36] Ramanathan, M.; Nettleton, E.; Darling, S. B. Thin Solid Films 2009, 517, $4474-4478$. 
[37] Cheng, J. Y.; Ross, C. A.; Chan, V. Z. H.; Thomas, E. L.; Lammertink, R. G. H.; Vancso, G. J. Adv. Mater. 2001, 13, 1174-1178.

[38] Lammertink, R. G. H.; Hempenius, M. A.; van den Enk, J. E.; Chan, V. Z. H.; Thomas, E. L.; Vancso, G. J. Adv. Mater. 2000, 12, 98-103.

[39] Suarez, M. F.; Compton, R. G. J. Electroanal. Chem. 1999, 462, 211-221.

[40] Smela, E.; Gadegaard, N. Adv. Mater. 1999, 11, 953-957.

[41] Schmidt, D. J.; Cebeci, F. C.; Kalcioglu, Z. I.; Wyman, S. G.; Ortiz, C.; Van Vliet, K. J.; Hammond, P. T. Acs Nano 2009, 3, 2207-2216.

[42] Lizarraga, L.; Andrade, E. M.; Molina, F. V. J. Electroanal. Chem. 2004, $561,127-135$.

[43] Wilbur, J. L.; Kumar, A.; Kim, E.; Whitesides, G. M. Adv. Mater. 1994, 6, 600-604.

[44] Xia, Y. N.; Kim, E.; Zhao, X. M.; Rogers, J. A.; Prentiss, M.; Whitesides, G. M. Science 1996, 273, 347-349.

[45] Zhao, X. M.; Xia, Y. N.; Whitesides, G. M. Adv. Mater. 1996, 8, 837-840.

[46] Kim, E.; Xia, Y. N.; Whitesides, G. M. Nature 1995, 376, 581-584.

[47] Kim, E.; Xia, Y. N.; Zhao, X. M.; Whitesides, G. M. Adv Mater 1997, 9, 651-654.

[48] Korczagin, I.; Lammertink, R. G. H.; Hempenius, M. A.; Golze, S.; Vancso, G. J. Adv. Polymer Sci. 2006, 200, 91-117.

[49] Song, J.; Janczewski, D.; Ma, Y. J.; van Ingen, L.; Sim, C. E.; Goh, Q. L.; Xu, J. W.; Vancso, G. J. Eur. Polym. J. 2013, 49, 2477-2484.

[50] Song, J.; Janczewski, D.; Guo, Y. Y.; Xu, J. W.; Vancso, G. J. Nanoscale 2013, 5, 11692-11698.

[51] Voskuhl, J.; Brinkmann, J.; Jonkheijm, P. Curr. Opin. Chem. Biol. 2014, $18,1-7$.

[52] Acikgoz, C.; Hempenius, M. A.; Huskens, J.; Vancso, G. J. Eur. Polym. J. 2011, 47, 2033-2052.

[53] Delamarche, E.; Schmid, H.; Bietsch, A.; Larsen, N. B.; Rothuizen, H.; Michel, B.; Biebuyck, H. J. Phys. Chem. B 1998, 102, 3324-3334.

[54] Delamarche, E.; Schmid, H.; Michel, B.; Biebuyck, H. Adv. Mater. 1997, 9, 741-746.

[55] Smith, R. K.; Lewis, P. A.; Weiss, P. S. Prog. Surf. Sci. 2004, 75, 1-68.

[56] Brandt, P. F.; Rauchfuss, T. B. J. Am. Chem. Soc. 1992, 114, 1926-1927. 
[57] Rulkens, R.; Lough, A. J.; Manners, I.; Lovelace, S. R.; Grant, C.; Geiger, W. E. J. Am. Chem. Soc. 1996, 118, 12683-12695.

[58] Shi, W. Q.; Giannotti, M. I.; Zhang, X.; Hempenius, M. A.; Scönherr, H.; Vancso, G. J. Angew. Chem. Int. Ed. 2007, 46, 8400-8404.

[59] Chao, T. S.; Lee, C. L.; Lei, T. F. J. Electrochem. Soc. 1991, 138, 1756-1761.

[60] Xia, Y. N.; Whitesides, G. M. Langmuir 1997, 13, 2059-2067.

[61] Kumar, A.; Biebuyck, H. A.; Whitesides, G. M. Langmuir 1994, 10, 14981511.

[62] Tien, J.; Xia, Y.; Whitesides, G. M. Thin Films 1998, 24, 227-250.

[63] Advincula, R.; Aust, E.; Meyer, W.; Knoll, W. Langmuir 1996, 12, 35363540 . 


\section{Chapter 4}

\section{Scanning near-field ellipsometric microscopy setup}

This chapter discusses the experimental arrangement of the scanning near-field ellipsometric microscopy (SNEM) setup established in this thesis, which is comprised of AFM combined with an ellipsometer. The AFM and the ellipsometer were both commercially available and they did not require major changes in their design to build the hybrid microscope. SNEM was used for optical imaging of heterogeneous transparent thin films with spatial resolution well below the diffraction limit. The point dipole model is introduced which explains the interactions between the coupled tip-sample dipole system in scattering near-field optical microscopy (s-SNOM). Additionally, the origins of the far-field background signal which depends interferometrically on the vertical tip position is explained. 
The contribution of this chapter is published in:

Cumurcu, A., Duvigneau, J., Lindsay, I. D., Schön, P. M., and Vancso, G. J., Eur. Polym. J. 94 (2013) 1935.

Cumurcu, A., Duvigneau, J., Lindsay, I. D., Schön, P. M., and Vancso, G. J., MRS Online Proceedings Library 1562 (2013) mrsf13-1652-ll06-03 doi:10.1557/opl.2014.261. 


\subsection{Introduction}

In the past 20 years, the atomic force microscope (AFM) has evolved into a powerful tool for the nanoscale investigation of polymeric materials down to the single macromolecule level [1]. Although AFM provides nanometer spatial resolution on topography, friction and mechanical properties, there is a fundamental need to obtain further material specific information, for instance with respect to optical material properties such as refractive index [2]. In this regard the integration of AFM into hybrid devices, in particular in combination with optical techniques, has opened novel avenues for nanoscale material characterization [3].

The introduction of tip-enhanced near-field optical techniques in the mid-1990s has brought simplicity and new possibilities with the use of already existing AFM and scanning tunneling microscopy (STM) probes [4-6]. These techniques rely on the strong electromagnetic field enhancement that occurs at the apex of the probing tip when appropriately illuminated by a light source. Therefore, the tip geometry, material, roughness and illumination parameters such as polarization state and angle of the incident light as well as the excitation wavelength affect the field enhancement [7-9]. Typically, the probing tip fulfills one or both of two roles. Firstly, the above mentioned field enhancement effects can cause the probe tip to act as a highly localized light source enhancing processes such as Raman scattering or fluorescence emission by the sample in the immediate vicinity. This is the basis of tip-enhanced near field optical microscopy techniques such as nearfield fluorescence enhancement [10], tip enhanced Raman scattering (TERS) [11], and tip-enhanced coherent anti-Stokes Raman scattering microscopy (CARS) [12]. Secondly, the probe tip can act as a localized sub-wavelength scatterer, effectively coupling the normally non-propagating evanescent field localized at the sample surface into the far field where it can be detected. This effect forms the basis of apertureless scanning near-field optical microscopy (a-SNOM), also termed scattering SNOM (s-SNOM), either with or without the additional field enhancement effect [13].

Karageorgiev et al. proposed a hybrid a-SNOM system consisting of a combination of an AFM with an ellipsometer to study the optical properties of thin polymeric films [14]. In this hybrid setup, termed scanning near-field ellipsometric microscopy (SNEM), the sample was illuminated from below through a prism in total internal reflection (TIR) to create an evanescent field that was scattered by an AFM probe tip at the sample surface. Hence, the instrument allowed imaging optical inhomogeneities in thin transparent films with a lateral resolution within the nanometer range. In this instrument, the AFM was operated in contact mode and by scanning the tip within the evanescent field, the null ellipsometry conditions were disturbed. Subsequently, the intensity change was recorded at the detector and displayed simultaneously with the topography image of AFM. A platinum coated silicon conical tip with a radius of $20 \mathrm{~nm}$ was used. The optical contrast occured due to the interference of the propagating waves generated in the near-field of the tip with the light reflected from the back surface of the film. 
Nine years after the introduction of SNEM, Liu et al. proposed a study on nearfield ellipsometry measurements to characterize dielectric constants and thicknesses of thin films using a home build system with an aperture fiber probe [15]. This SNEM was operated at transmission configuration and the incident angle was adjusted to achieve total internal reflection to create evanescent waves on the top surface of the thin film. The heterodyne detection was used to measure the near-field polarization for $x$ and $y$ polarized light on the thin film surface. More recently, they presented a new version of the setup which works in the reflection configuration [16]. This method allows the characterization of optical properties of samples on bulky or opaque substrates. Additionally, new ellipsometry equations to achieve more precise thin film characterization were established by including the near-field sample-probe interactions.

In this thesis, an alternative SNEM configuration, which relies on recording the changes in the state of the polarization of the electromagnetic field scattered by the vibrating probe placed in the proximity of the specimen surface, is used [17-19]. For excitation of gold-coated probes in the visible region of the spectrum, as used here, field enhancement at the tip apex would be expected to occur both as a result of the increasing confinement of the surface charge density at the sharp tip apex, the so-called "lightning-rod effect" and due to the resonant excitation of localized surface plasmon modes of the metalized probe tip [20-22]. Such an enhanced electromagnetic field at the tip apex is altered by the presence of the sample. Therefore, in our setup, the signal-to-noise ratio is increased compared to the first SNEM setup from Karageorgiev in which the probe is used solely to scatter the evanescent field at the sample surface. It should also be noted that depending on the mode of operation of the AFM other surface specific information, e.g. phase and friction contrast, can be obtained as well.

Qualitative calculation of the relative scattering when probing different materials was suggested with a simple model called point dipole model [23-25]. In this model, the scattering probe was assumed to be a polarizable sphere. The electric field of the induced probe dipole was assumed to induce surface charges in the sample creating an image dipole located inside the sample. Hence, the effective polarizability of the probe-sample coupled system was defined by the superposition of the both probe and image dipole fields. This model was most commonly used to explain material contrast observed in SNOM experiments [9, 26-33]. However, the point dipole model fails to provide quantitative description of the near-field contrast, mainly since the tip shape is simplified to a sphere.

A model called finite-dipole model, which represents the probe as an isolated spheroid, was then introduced to improve the analytical model of the near-field interactions [34]. Numerical methods namely 2D finite element calculations (FEM), boundary element method (BEM) and finite difference time-domain (FDTD) method were also used to describe near-field interactions [35-37]. However, since the point dipole model is a simple and efficient model for qualitative comparisons of the experimental results, it was used in this thesis to explain some of the phenomena observed in SNEM measurements. 
In this chapter, introductions of the experimental arrangement of the SNEM setup and the point dipole model are provided. Also the origin of the far-field background signal is explained.

\subsection{Principle of the SNEM setup}

SNEM is based on recording the changes in the state of polarization of the light. The incident beam contains electric fields both parallel $(p-)$ and perpendicular $(s-)$ to the plane of incidence. The tip-sample coupled system changes the $p-$ and $s$ - polarization of the light resulting to a change in the outgoing polarization of the light. Since the dielectric constant of the tip is consistent during scanning, the variations in the dielectric constant of the sample play the major role in changing the $p-$ and $s$ - polarization of the light. Therefore, SNEM is sensitive to the differences in the dielectric constants of the different domains present in the sample.

The ellipsometric contrast mechanism of SNEM resembles the mechanism of imaging ellipsometry (a study as representative example of imaging ellipsometry is given in Chapter 3) providing sub-nanometer vertical sensitivity [38, 39]. By SNEM one can measure surface heterogeneities with small differences in their dielectric constants, e.g. between the blocks of block copolymers [17, 18]. The advantage of SNEM over imaging ellipsometry is its nanoscale resolution in the lateral direction. In contrast, the lateral resolution (approximately $2 \mu \mathrm{m}$ ) of imaging ellipsometry is limited to the wavelength of the light source and the numerical aperture of the objective used.

Fig. 4.1 shows a schematic representation of the SNEM hybrid setup. The AFM probe tip is illuminated from below by elliptically polarized laser light of the ellipsometer at an incident angle $\theta$ smaller than the critical angle. Imaging is carried out by raster-scanning the transparent glass-substrate supported samples in the $x$ - $y$ direction, while the AFM probe position is controlled in the $z$-direction to accommodate the sample topography. This configuration is advantageous for near-field optical microscopy applications, since the realignment of the laser is not necessary.

The AFM is operated in tapping mode AFM imaging, while contact mode AFM imaging was used in a previous work on the same setup performed in our laboratory [18]. Intermittent contact or tapping mode imaging reduces the probability of tip wear and contamination significantly due to greatly reduced lateral forces between the AFM probe tip and substrate surface. In addition, the use of tapping mode imaging allows the characterization of a broader range of samples that cannot be addressed by contact mode imaging due to e.g. plastic deformation of the sample surface. Furthermore, recording the phase of the probe oscillations provides an additional mechanical contrast mechanism [40, 41]. 


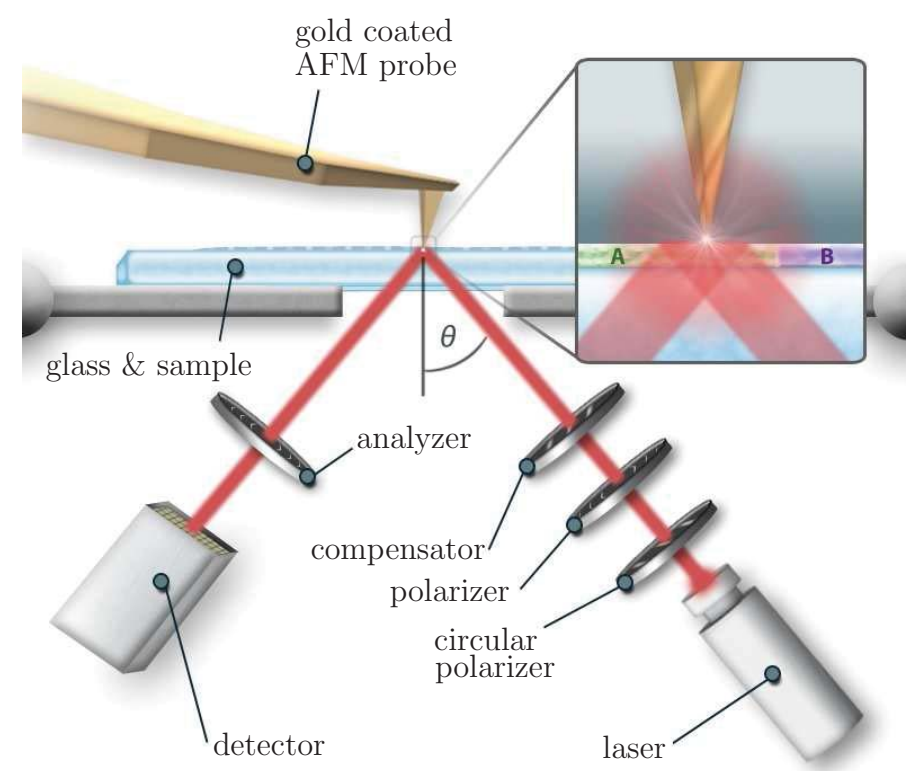

Figure 4.1: Schematic representation of the SNEM setup, a hybrid microscope that consists of a commercially available AFM and ellipsometer. In our AFM instrument, a decoupled $X-Y$ and $Z$ scanner is used. The AFM tip is illuminated from below with polarized laser light of the ellipsometer operated at null conditions. The ellipsometer laser light has an incident angle of $40^{\circ}$. The inset demonstrates the electromagnetic field enhancement at the tip apex.

Prior to scanning, when the tip is in contact with the sample surface, nullellipsometry conditions are applied. This procedure consists of finding component (polarizer and analyzer) settings that minimize the light at the detector. The azimuths of the polarizer and compensator are adjusted to achieve the correct elliptical polarization which, following reflection from the sample surface, results in linearly polarized light. The analyzer is then set perpendicular to the polarization axis of the linearly polarized reflected light to minimize the intensity at the detector.

More precisely, the net polarization state resulting from the superposition of the near field local scattering of the AFM probe and the scattered field averaged over the whole illuminated area of the sample (which also includes the background scattering which originates from scattering between the AFM cantilever and the sample surface) is minimized at the detector for the first scan point by applying null-ellipsometry conditions when the AFM tip is in contact with the surface. However, since the polarization state of the light scattered by the tip is different at different locations on the heterogeneous sample, the initially set null-ellipsometry conditions are altered during scanning. These changes in the polarization state are detected at the ellipsometer detector for each scanned point. 
As already noted, two mechanisms contribute to the electromagnetic field enhancement in the immediate vicinity of the probing tip: surface plasmon resonances and the lighting rod effect. The first mechanism is based on the excitation of localized surface plasmon resonances at the metal coated AFM tip by the incident light. Approximating the end of the probing tip to a metallic spheroidal particle allows a resonance condition to be defined which depends on the spheroid geometry and dielectric constant [42]. Excitation with an incident field at this resonant frequency thus leads to strong field enhancement at the tip apex.

(a)

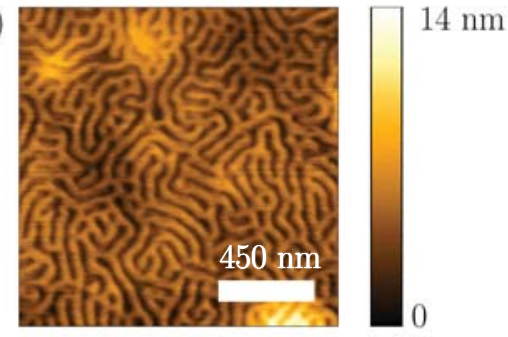

(b)

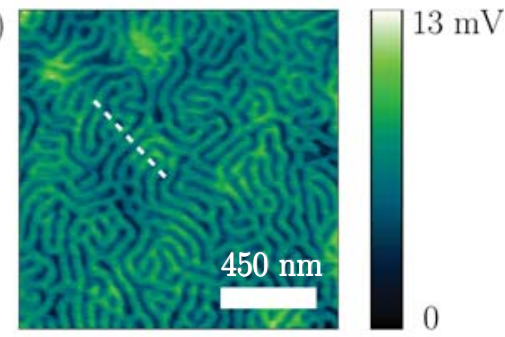

(c)

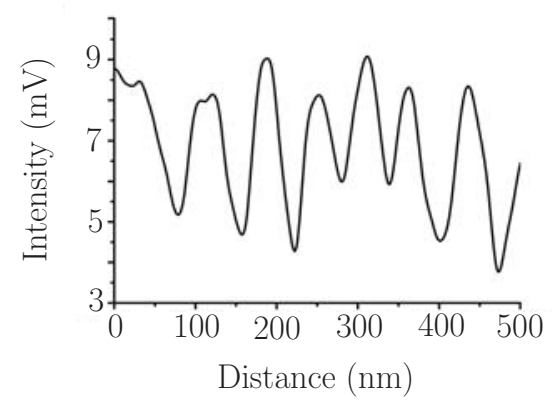

Figure 4.2: SNEM data of a microphase separated PS- $b$-PMMA diblock copolymer film: (a) AFM height, (b) corresponding optical image. In (c), the corresponding cross-section along the white dashed line in (b) is shown for the optical image. The image is collected in tapping mode AFM at $0.75 \mu \mathrm{m} \mathrm{s}^{-1}$ with $1024 \times 1024$ pixel $^{2}$ resolution.

The latter mechanism corresponds to local field enhancement by a nonresonant effect. The electromagnetic field is highly enhanced at any sharp geometrical feature as charge tends to accumulate at a sharper edge [43]. Therefore, sharp objects with a high aspect ratio will generate a strong field. Consequently, the used material, size, shape and roughness of the tip as well as the properties of the excitation light source such as the wavelength and the polarization play an important role in the field enhancement [11]. An example material used in SNEM optical imaging is a microphase separated PS- $b$-PMMA diblock copolymer film. Fig. 4.2 shows a tapping mode AFM height and a corresponding SNEM optical image of this block copolymer film. The optical image revealing strong contrast allows the typical microphase separated morphology to be resolved. The average spacing of the lamellar structure of the block copolymer is $37 \mathrm{~nm}$. 


\subsection{Point dipole model}

A simple model termed point dipole model describes the optical near-field interactions between the probing tip and the sample surface $[23,25,26,44,45]$. In this model, the scattering probe is assumed to be a polarizable sphere with dielectric value $\varepsilon_{t}$, radius $a \ll \lambda$ and polarizability of

$$
\alpha=4 \pi a^{3} \frac{\left(\varepsilon_{t}-1\right)}{\left(\varepsilon_{t}+2\right)} .
$$

The scattering probe is induced by the incident field $E_{i}$ in $z$-direction, as suggested by the antenna action of the shaft of the tip (Fig. 4.3). The sample, with a dielectric constant $\varepsilon_{s}$ and polarizability of

$$
\beta=\frac{\left(\varepsilon_{s}-1\right)}{\left(\varepsilon_{s}+1\right)}
$$

is assumed to be polarizable only by the sphere's dipolar field, since the enhanced near-field at the tip exceeds the incident field, $E_{i}$. Additionally, the probe's sphere is replaced by a point dipole of equal strength (direction is located in the sphere's center) to simplify the calculations.

The response of the sample to the sphere's point dipole is a mirror charge inside the sample with the polarizability of $\alpha \beta$. The electric field generated by the mirror dipole acts back on the sphere's point dipole, decreasing with the third power of distance, and further polarizes it. Multiple interactions resulting from the response of the sample to the sphere's point dipole are described by the effective polarizability, $\alpha_{e f f}$, according to eq. 4.3 :

$$
\alpha_{e f f}=\frac{\alpha(1+\beta)}{1-\frac{\alpha \beta}{16 \pi(a+z)^{3}}},
$$

where $z$ is the tip-sample separation. The scattered field, $E_{s}$, from the coupled tipsample dipole system is directly proportional to the effective polarizability $E_{s} \propto$ $\alpha_{e f f} E_{i}$. The effective polarizability is a complex value which can be expressed as

$$
\alpha_{e f f}=s e^{i \varphi}
$$

by a relative amplitude s and phase shift $\varphi$ between the incident and the scattered light. Eq. 4.3 shows that a sample influences the scattered near-field signal only through its dielectric value $\varepsilon_{s}$ at the wavelength of illumination. This reveals that s-SNOM can provide local dielectric identification of heterogeneous sample systems. 


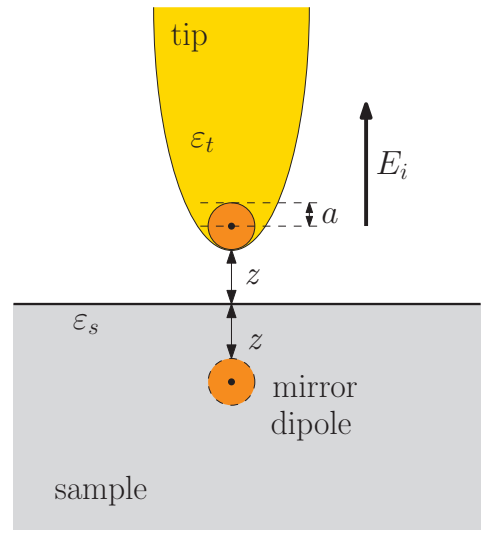

Figure 4.3: Model of the near-field interactions between coupled tip-sample dipole systems in s-SNOM.

\subsection{Contribution of the far-field signal}

In SNEM, the laser light illuminates not only the region of the tip apex but also a wider region of the surface and the tip shaft and cantilever (Fig. 4.4). Light scattered from these unwanted sources combine to create a far-field background signal that depends interferometrically on the vertical tip position [6]. Initially the background scattering is minimized in SNEM by setting the null conditions. However, during scanning the background signal varies with the motion of the tip normal to the sample surface (on scale $\lambda$ due to interference fringes parallel to the sample surface) introducing a cross-talk between height and optical images. This is termed $z$-motion artifact, and it yields features in the optical image that are correlated to the structures in the sample topography, thus making image interpretation ambiguous [46-48].

An intensity-distance curve, collected with a gold coated AFM probe tip on a PSb-PMMA block copolymer film, is shown in Fig. 4.5. This curve reveals oscillations which originate from the interference of the multiple reflections between the AFM tip, including the tip shaft, and the sample surface. In this respect, the tipsample system resembles the two moving mirrors in a Michelson interferometer with a periodicity of $\lambda /(2 \cos \theta)$ equal to $413 \mathrm{~nm}$ (incident angle of the light, $\left.\theta=40^{\circ}\right)[46,49]$.

Additionally, the initially nulled area which is illuminated by the laser light changes during scanning since a $X-Y$ sample scanner is used. This deviation from the nulled area can introduce a change in the background signal as well. However, for samples with relatively uniform distribution of features that are much smaller than the focus spot, this effect expected to be relatively small as the changes are averaged over a large area. 


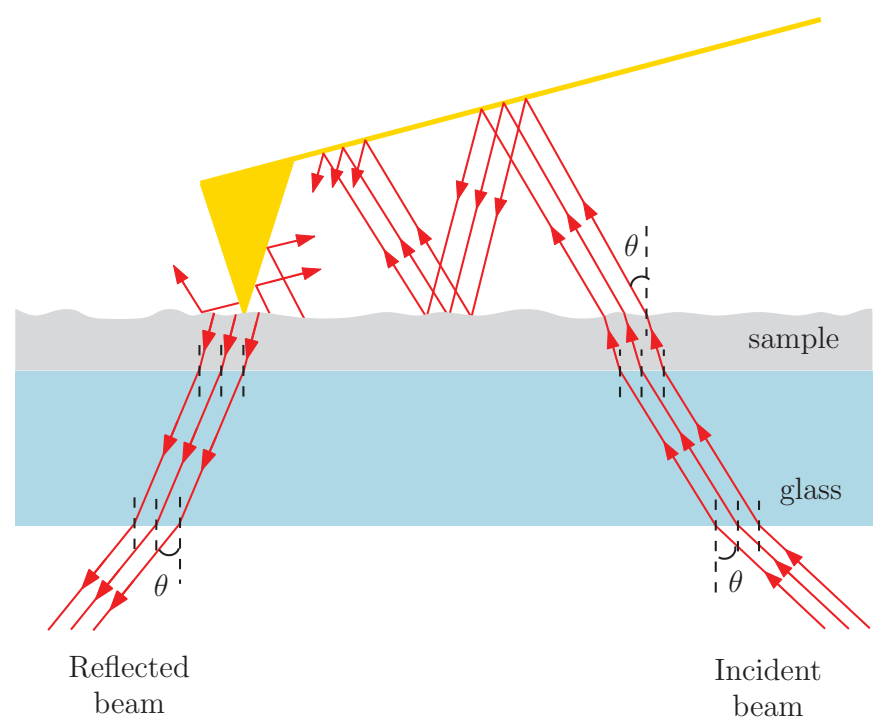

Figure 4.4: The optical path and the multiple reflections between the gold coated AFM cantilever and the sample surface. The angle of the incident beam is $\theta$. For the clarity of the image the complete light path is not shown.

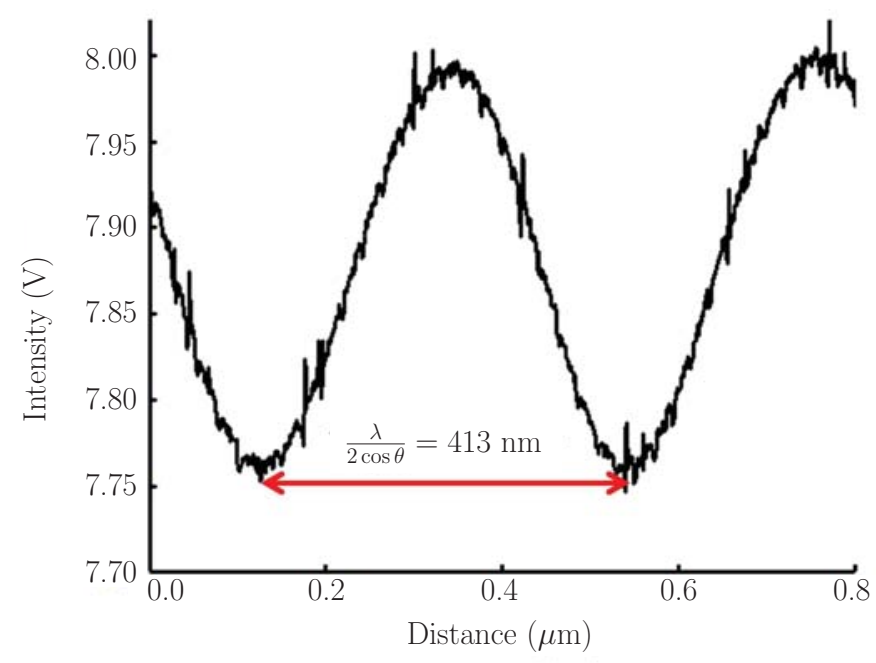

Figure 4.5: A SNEM intensity-distance curve recorded simultaneously during acquisition of a force curve on a polymer film. The optical signal shows oscillations with $\lambda /(2 \cos \theta)$ period. 


\subsection{Conclusions}

This chapter described the experimental arrangement of the SNEM setup which consists of an AFM and an ellipsometer for optical imaging of heterogeneous transparent thin films. The point dipole model which describes the interactions between the coupled tip-sample dipole system in s-SNOM was deliberated. Additionally, the far-field background signal, especially its origin and its effect to the optical image, was discussed.

\subsection{Experimental section}

Materials: PS- $b$-PMMA diblock copolymer with a number average molar mass, $\mathrm{M}_{n}$, of 50000 and $47000 \mathrm{~g} \mathrm{~mol}^{-1}$ for the PS and PMMA block, respectively and a polydispersity index of 1.09 was obtained from Polymer Source Inc. (Montreal, Canada). Toluene was purchased from Biosolve (Valkenswaard, the Netherlands). All compounds were used without further purification. Milli-Q water was produced by a Millipore Synergy system (Billereca, MA, USA). The glass slides were purchased from Thermo Scientific (Menzel-Glaser, Gerhard Menzel GmbH, Braunschweig, Germany). The glass slides were made of extra-white soda-lime glass. Root mean square (RMS) roughness of a glass slides was $0.77 \mathrm{~nm}$ for a $1.5 \times 1.5 \mu \mathrm{m}^{2}$ area as determined by AFM.

Film preparation: The PS- $b$-PMMA diblock copolymer was dissolved in toluene (1 wt.\% solution) and spin coated on a piranha cleaned glass slide at $2000 \mathrm{rpm}$ for 1 minute. Prior to use, the glass slide with size of $26 \times 76 \times 1.0 \mathrm{~mm}$ and refractive index of 1.5 was cleaned in piranha solution $\left(1: 3(\mathrm{v} / \mathrm{v})\right.$ solution of $30 \% \mathrm{H}_{2} \mathrm{O}_{2}$ an concentrated $\mathrm{H}_{2} \mathrm{SO}_{4}$ ) for 20 minutes, rinsed with copious amounts of MilliQ water and ethanol followed by drying at $\mathrm{N}_{2}$ flow. Caution: Piranha solution is highly corrosive. Extreme care should be taken when handling Piranha solution and only small quantities should be prepared. The spin coated polymer film was annealed in air at $175^{\circ} \mathrm{C}$ overnight $[50,51]$. The film thickness was $35 \mathrm{~nm}$, as determined by AFM height measurements.

SNEM: The MFP-3D AFM (Asylum Research, Santa Barbara, CA, USA) was combined with a Multiskop ellipsometer (Optrel, Sinzing, Germany) in the SNEM setup. The sample was placed on a custom-made interface plate (Optrel, Sinzing, Germany). The ellipsometer was equipped with a HeNe laser $(\lambda=632.8 \mathrm{~nm})$ and was operated at an angle of incidence of $40^{\circ}$. The ellipsometer was operated at the null settings controlled by means of a computer with a sensitivity of $1 / 1000$ of a degree. For the SNEM measurement in tapping mode AFM imaging, a silicon cantilever (PointProbe Plus silicon probes, PPP-NCH, Nanosensors, Neuchatel, Switzerland) with a nominal resonance frequency of approximately $330 \mathrm{kHz}$ and a spring constant of approximately $42 \mathrm{~N} \mathrm{~m}^{-1}$ was used. The probe was coated with a $13 \mathrm{~nm}$ thin layer of gold using a Balzers SCD 040 sputtering machine at 
an argon pressure of 0.05 mbar (250 sec sputtering at a $15 \mathrm{~mA}$ current). The tip was vibrated perpendicularly to the sample surface with amplitude of approximately $32 \mathrm{~nm}$ (i.e. $64 \mathrm{~nm}$ peak to peak) [6, 49]. The amplitude setpoint was maintained by a feedback loop at approximately $80 \%$ of the free oscillation amplitude. The SNEM optical images were processed by polynomial background leveling, followed by two-dimensional fast Fourier transform (2D-FFT) filtering, to remove unwanted periodic structures attributed to electronic noise at fixed frequencies using Gwyddion software [52]. Images were collected in air at scan rates of $0.75 \mu \mathrm{m} \mathrm{s}^{-1}$ with $1024 \times 1024$ pixel $^{2}$ resolution. 


\section{Bibliography}

[1] Schönherr, H.; Vancso, G. J. Scanning force microscopy of polymers; Springer: Berlin Heidelberg, 2010.

[2] Redondo-Morata, L.; Giannotti, M. I.; Sanz, F. Molec. Membrane Biol. 2014, 31, 17-28.

[3] Flores, S. M.; Toca-Herrera, J. L. Nanoscale 2009, 1, 40-49.

[4] Inouye, Y.; Kawata, S. Opt. Lett. 1994, 19, 159-161.

[5] Zenhausern, F.; O'boyle, M. P.; Wickramasinghe, H. K. Appl. Phys. Lett. 1994, 65, 1623-1625.

[6] Bachelot, R.; Gleyzes, P.; Boccara, A. C. Opt. Lett. 1995, 20, 1924-1926.

[7] Adam, P. M.; Royer, P.; Laddada, R.; Bijeon, J. L. Appl. Optics 1998, 37, 1814-1819.

[8] Adam, P. M.; Royer, P.; Laddada, R.; Bijeon, J. L. Ultramicroscopy 1998, 71, 327-331.

[9] Raschke, M. B.; Lienau, C. Appl. Phys. Lett. 2003, 83, 5089-5091.

[10] Frey, H. G.; Paskarbeit, J.; Anselmetti, D. Appl. Phys. Lett. 2009, 94, 241116 1-3.

[11] Bailo, E.; Deckert, V. Chem. Soc. Rev. 2008, 37, 921-930.

[12] Ichimura, T.; Hayazawa, N.; Hashimoto, M.; Inouye, Y.; Kawata, S. Phys. Rev. Lett. 2004, 92, 220801 1-4.

[13] Keilmann, F. J. Electron Microsc. 2004, 53, 187-192.

[14] Karageorgiev, P.; Orendi, H.; Stiller, B.; Brehmer, L. Appl. Phys. Lett. 2001, 79, 1730-1732.

[15] Liu, Z.; Zhang, Y.; Kok, S. W.; Ng, B. P.; Soh, Y. C. Opt. Express 2010, 18, 3298-3310.

[16] Liu, Z.; Zhang, Y.; Kok, S. W.; Ng, B. P.; Soh, Y. C. Ultramicroscopy 2013, 124, 26-34.

[17] Cumurcu, A.; Duvigneau, J.; Lindsay, I. D.; Schön, P. M.; Vancso, G. J. Eur. Polym. J. 2013, 49, 1935-1942.

[18] Tranchida, D.; Diaz, J.; Schön, P.; Schönherr, H.; Vancso, G. J. Nanoscale 2011, 3, 233-239.

[19] Cumurcu, A.; Duvigneau, J.; Lindsay, I. D.; Schön, P. M.; Vancso, G. J. MRS Online Proceedings Library 2013, 1562. 
[20] Asghari-Khiavi, M.; Wood, B. R.; Hojati-Talemi, P.; Downes, A.; McNaughton, D.; Mechler, A. J. Raman Spectrosc. 2012, 43, 173-180.

[21] Hecht, B.; Sick, B.; Wild, U. P.; Deckert, V.; Zenobi, R.; Martin, O. J. F.; Pohl, D. W. J. Chem. Phys. 2000, 112, 7761-7774.

[22] Novotny, L.; van Hulst, N. Nat. Photonics 2011, 5, 83-90.

[23] Knoll, B.; Keilmann, F. Nature 1999, 399, 134-137.

[24] Hillenbrand, R.; Knoll, B.; Keilmann, F. J. Microsc-Oxford. 2001, 202, 7783.

[25] Keilmann, F.; Hillenbrand, R. Philos T Roy Soc A 2004, 362, 787-805.

[26] Taubner, T.; Hillenbrand, R.; Keilmann, F. Appl. Phys. Lett. 2004, 85, $5064-5066$.

[27] Raschke, M. B.; Molina, L.; Elsaesser, T.; Kim, D. H.; Knoll, W.; Hinrichs, K. Chemphyschem. 2005, 6, 2197-2203.

[28] Brehm, M.; Taubner, T.; Hillenbrand, R.; Keilmann, F. Nano. Lett. 2006, 6, 1307-1310.

[29] Ocelic, N.; Hillenbrand, R. Nat. Mater. 2004, 3, 606-609.

[30] Huber, A.; Ocelic, N.; Taubner, T.; Hillenbrand, R. Nano. Lett. 2006, 6, $774-778$.

[31] Samson, J. S.; Wollny, G.; Brundermann, E.; Bergner, A.; Hecker, A.; Schwaab, G.; Wieck, A. D.; Havenith, M. Phys. Chem. Chem. Phys. 2006, $8,753-758$.

[32] Kim, Z. H.; Liu, B.; Leone, S. R. J. Phys. Chem. B 2005, 109, 8503-8508.

[33] Hillenbrand, R.; Keilmann, F. Appl. Phys. Lett. 2002, 80, 25-27.

[34] Cvitkovic, A.; Ocelic, N.; Hillenbrand, R. Appl. Phys. Lett. 2007, 15, 85508565 .

[35] Fikri, R.; Barchiesi, D.; H'Dhili, F.; Bachelot, R.; Vial, A.; Royer, P. Opt. Commun. 2003, 221, 13-22.

[36] Cvitkovic, A.; Ocelic, N.; Aizpurua, J.; Guckenberger, R.; Hillenbrand, R. Phys. Rev. Lett. 2006, 97, 060801 1-4.

[37] Hatano, H.; Kawata, S. J. Microsc-Oxford. 1999, 194, 230-234.

[38] Bae, Y. M.; Oh, B. K.; Lee, W.; Lee, W. H.; Choi, J. W. Biosens. Bioelectron. 2004, 20, 895-902.

[39] Harke, M.; Motschmann, H. Langmuir 1998, 14, 313-318. 
[40] Reynaud, C.; Sommer, F.; Quet, C.; El Bounia, N.; Duc, T. M. Surf. Interface Anal. 2000, 30, 185-189.

[41] Tomasetti, E.; Legras, R.; Nysten, B. Nanotechnology 1998, 9, 305-315.

[42] Zayats, A. V. Opt. Commun. 1999, 161, 156-162.

[43] Yang, Z. L.; Aizpurua, J.; Xu, H. X. J. Raman Spectrosc. 2009, 40, 13431348.

[44] Knoll, B.; Keilmann, F. Opt. Commun. 2000, 182, 321-328.

[45] Hillenbrand, R.; Taubner, T.; Keilmann, F. Nature 2002, 418, 159-162.

[46] Weston, K. D.; Buratto, S. K. J. Phys. Chem. B 1997, 101, 5684-5691.

[47] Hecht, B.; Bielefeldt, H.; Inouye, Y.; Pohl, D. W.; Novotny, L. J. Appl. Phys. 1997, 81, 2492-2498.

[48] Kaupp, G.; Herrmann, A.; Haak, M. J. Phys. Org. Chem. 1999, 12, 797-807.

[49] David, T.; Chicanne, C.; Richard, N.; Krenn, J. R.; Scheurer, F.; Ounadjela, K.; Hehn, M.; Lacroute, Y.; Goudonnet, J. P. Rev. Sci. Instrum. 1999, 70, 4587-4594.

[50] Morkved, T. L.; Jaeger, H. M. Europhys. Lett. 1997, 40, 643-648.

[51] Zhang, X. H.; Berry, B. C.; Yager, K. G.; Kim, S.; Jones, R. L.; Satija, S.; Pickel, D. L.; Douglas, J. F.; Karim, A. Acs Nano 2008, 2, 2331-2341.

[52] Necas, D.; Klapetek, P. Cent. Eur. J. Phys. 2012, 10, 181-188. 


\section{Chapter 5}

\section{Multimodal imaging of PS- $b$-P2VP block copolymer films}

Scanning near-field ellipsometric microscopy (SNEM) is considered in this chapter to simultaneously obtain optical and tapping mode AFM topography images of a microphase separated morphology of poly(styrene- $b$-2-vinyl pyridine) (PS- $b$-P2VP) block copolymer thin films. Optical images revealed a spatial resolution of approximately $80 \mathrm{~nm}$. To obtain a deeper insight into the nature of the SNEM contrast mechanism, the use of gold coated and bare silicon AFM probe tips were investigated for the SNEM measurements. In addition, the dielectric constant difference between the two blocks of the diblock copolymer was increased by selectively staining the P2VP block with iodine vapor. This resulted in an increase in the optical contrast between the PS and P2VP domains. Furthermore, AFM probe tip-sample distances were varied and the resulting change in optical images was elucidated. Calculations according to the point dipole model for a polarizable probe in proximity to a sample surface supported the experimental data. 
The contribution of this chapter is published in:

Cumurcu, A., Duvigneau, J., Lindsay, I. D., Schön, P. M., and Vancso, G. J., Eur. Polym. J. 94 (2013) 1935.

Cumurcu, A., Duvigneau, J., Lindsay, I. D., Schön, P. M., and Vancso, G. J., MRS Online Proceedings Library 1562 (2013) mrsf13-1652-ll06-03 doi:10.1557/opl.2014.261. 


\subsection{Introduction}

Since the mid-1990s, apertureless scanning near-field optical microscopy (a-SNOM) has branched into a variety of powerful techniques, for the optical characterization of sample surfaces with resolution far below the diffraction limit. Tip enhanced fluorescence microscopy (TEFM) [1], tip enhanced Raman scattering (TERS) [2], tip-enhanced coherent anti-Stokes Raman scattering microscopy (CARS) [3], and scanning near-field ellipsometric microscopy (SNEM) [4], are examples of these techniques.

Metallic probe tips are frequently used in a-SNOM experiments because of their high scattering efficiency and field enhancement effects [2, 5-7]. Commonly, the etched metal wires and metal coated scanning probe microscopy (SPM) probes are employed due to the simplicity of their production $[2,7]$. The characteristics of the tips such as the tip geometry, its material and dielectric function have a major effect on the efficient coupling of light with the optical probe and thus the near-field optical enhancement.

Silver and gold metal coatings are most frequently used in TERS due to their high resonances in the visible light range [7]. The excitation of localized surface plasmon resonances at the metal coated tips enhances the electromagnetic field in the vicinity of the probe tip [8]. Gold reveals strong resonances above $600 \mathrm{~nm}$, while silver exhibits plasmonics over the entire visible range [7]. Other metals such as platinum, palladium and aluminum are less suitable, because they absorb strongly in the visible region. In a study, characteristic local field distributions and the corresponding field enhancement near the apex regions of four free standing tips with the same radius and shape but different materials, namely gold, tungsten, silicon and glass, were calculated within the quasistatic approximation [9]. These calculations have shown that the strongest field enhancement for the incident field with a wavelength of $630 \mathrm{~nm}$ was provided by the gold tip followed by tungsten, silicon and glass.

A TERS study on hematin samples using silver coated tips with coating thicknesses of 20,30 and $60 \mathrm{~nm}$ showed that the efficiency of the TERS probes were related to the total metal surface coverage [5]. When the cantilever was coated with a thick layer $(60 \mathrm{~nm})$ of silver, nanoparticle clusters on the tip apex affected the spatial resolution of the probe resulting in an inefficient probe. In the case of the thin silver layer $(20 \mathrm{~nm})$, the isolated metal islands were created and therefore, the yield of the probe was low. Finally, the medium thickness layer $(30 \mathrm{~nm})$ of silver resulted in a high efficiency probe for TERS.

The field enhancement at the apex of a gold-coated probe in the visible region of the spectrum would be expected to arise due to both a resonant and nonresonant excitation [5, 10-12]. The excitation of the localized surface plasmon polaritons by an external electric field enhances the incident field at the tip apex [5, 8]. The local field enhancement can also be achieved by a nonresonant effect, the 
so-called lightning-rod effect [5]. The sharp corner or the edge of a metal or the semiconductor object enhances the electromagnetic field due to the accumulation of dipolar fields. Therefore sharper objects with a higher aspect ratio create stronger field enhancement.

The local field enhancement is also depending on the distance between the tip and the sample. A study by Behr et al. revealed the variation of field enhancement at different tip-sample distances for a gold tip approaching to a gold sample surface [9]. The simulation results, based on the quasistatic approximation, suggested that the tip-sample interactions are related to the tip radius and become substantial at distances below the tip diameter. The field enhancement was found to rise superexponentially (faster than exponential increase) below distances equal to the tip radius and reach the maximum at the tip-sample contact. This nonlinear dependency of the scattering amplitude to the tip-sample distance is typical for near-field interactions [13].

Block copolymers are particularly suitable to investigate the lateral optical resolution of our SNEM setup due to the small scale of the phase separation (typically between 10-100 nm) between the blocks of polymers with different refractive index $[12,14,15]$. Repulsion between the domains of a block copolymer lead to segregation of the domains. However, due to the chemical bonding between the domains, the segregation cannot lead to macroscopic phase separation as in mixtures of two homopolymers. Instead there is a microphase separation that consists of microdomains rich in the blocks of the polymer [16-18]. In case of the microphase separations, these microdomains form a regular arrangement leading to a periodic structures such as the film morphology and the structural orientation of the block copolymer thin films are determined by various molecular and operational parameters such as block compositions, molecular weights and interfacial interactions with substrate and air [19].

Multimodal imaging of microphase separated poly(styrene- $b$-2-vinyl pyridine) (PS$b$-P2VP) block copolymer films by atomic force microscopy (AFM) and SNEM were reported in this chapter. Optical images captured with both gold coated and bare silicon AFM probe tips were investigated to obtain better understanding of the optical signal enhnacement in SNEM. The dielectric constant difference between the two blocks of the diblock copolymer was increased by selectively staining the P2VP block with iodine vapor. This resulted in an increase in the amplitude of the intensity variations between the PS and P2VP domains. Furthermore, the effect of increasing tip-sample separation on the amplitude of the intensity variations was studied. The non-linear decay of the near-field amplitude signal as a function of the tip-sample separation calculated using the point dipole model supported this experimental result. 


\subsection{Results and discussion}

\subsubsection{Comparing the gold coated tip with a bare silicon tip}

The microphase separated morphology of a PS- $b$-P2VP polymer thin film is imaged with SNEM operating the AFM in tapping mode. Fig. 5.1 shows AFM height, (a) and (c), and corresponding optical images, (b) and (d), collected with gold coated and bare silicon AFM probe tips, respectively. The optical image recorded with the gold coated tip reveals the P2VP vertical cylindrical domains within the PS matrix (Fig. 5.1(b)). The P2VP domains exhibited a lower optical signal than the surrounding PS matrix. The observed morphology matches well with the morphology observed in the corresponding topography image (Fig. 5.1(a)). In great contrast, the optical image recorded with the bare silicon tip shows almost no features characteristic for the microphase separated morphology.

The SNEM optical images were compared according to the amplitude of the intensity variations, calculated by the root mean square (RMS) deviation from the mean. It is important to note that the average optical intensities of the SNEM optical images may vary depending on the alignment of the tip and the nulling conditions. The amplitudes of the intensity variations arising from the periodic domain pattern form by the diblock copolymer were 1.0 and $0.2 \mathrm{mV}$ for the images captured with gold coated and bare silicon tip, respectively. This means that the field enhancement decreases by a factor 5 if a silicon tip used instead of a gold tip. The observed difference in the amplitude of the intensity variations was mainly attributed to the localized field enhancement due to a stronger lightning rod effect, the surface plasmon excitation and the higher scattering efficiency of the gold coated AFM probe compared to the bare silicon tip [9]. These results were in good agreement with data reported for gold-coated, silver-coated and uncoated tips used in tip-enhanced Raman microscopy [20]. The study demonstrated that the optical scattering spectra for isolated metal-coated tips showed wavelength dependence indicative of resonant excitation in the visible region, whereas the uncoated tips revealed a flat spectrum with a low signal intensity.

The laser light illuminates not only the apex of the AFM tip but also a large surrounding area which includes parts of the cantilever. Multiple reflections between the tip and the sample results in a background signal which is intially nulled in SNEM. The details of the background signal is discussed in Chapter 4. Due to the roughness of the sample surface, the background signal deviate from the null conditions resulting in an optical signal which is correlated to the height variations on the surface. In this regard, it is important to note that in addition to the contribution of the near-field enhancement effect of the gold coating on the optical images as obtained in Fig. 5.1, the reflections from the cantilever during raster scanning with the tip may contribute stronger for the gold coated tip than for the bare silicon tip. 


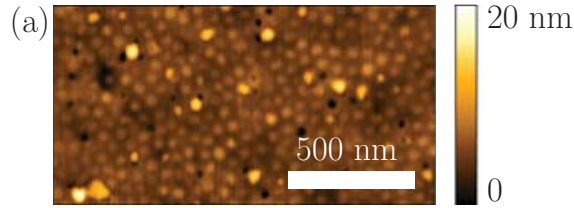

(c)

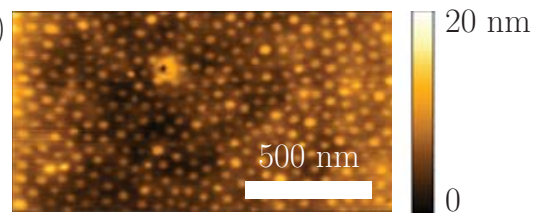

(e)

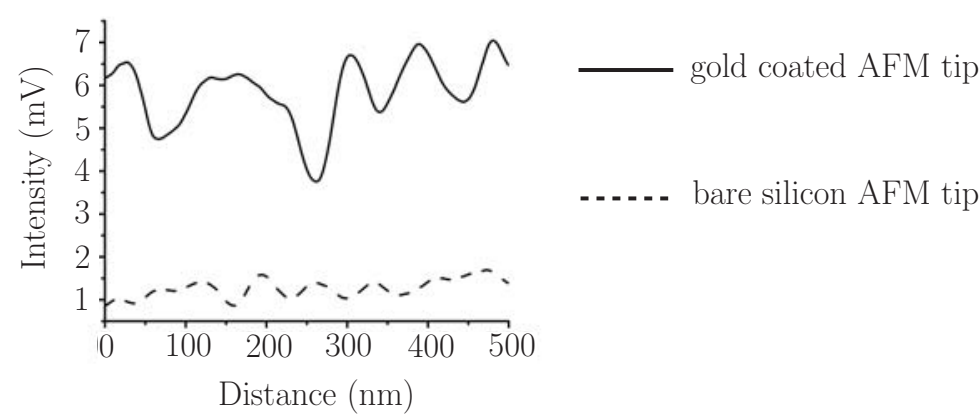

(b)

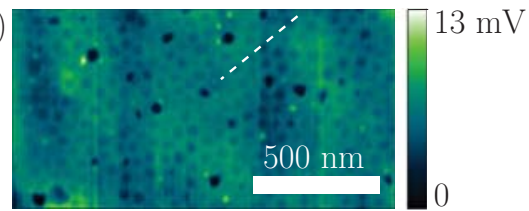

(d)

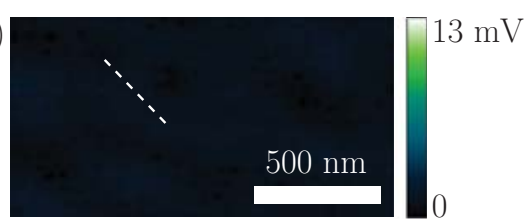

$3 \mathrm{mV}$

Figure 5.1: SNEM data of a microphase separated PS- $b$-P2VP diblock copolymer film: (a) AFM height and (b) corresponding optical image collected by a gold coated AFM tip, (c) AFM height and (d) corresponding optical image collected by a bare silicon AFM tip. In (e) the corresponding intensity cross sections along the white dashed lines for optical images (b) and (d) are shown. Images were collected at $0.4 \mu \mathrm{m} \mathrm{s}^{-1}$ with $1024 \times 683$ pixels resolution.

Furthermore, it is obvious from the height images shown in Fig. 5.1 that the P2VP domains are better resolved with the bare silicon tip compared to the gold coated tip. This was ascribed to the increase in AFM tip radius upon coating the tip with a gold layer of approximately $13 \mathrm{~nm}$ thickness. This brings forward a crucial point for the preparation and selection of the AFM probe tips that are used for SNEM, in analogy to those that are used in TERS studies [21]. Choosing the optimum coating thickness is important in obtaining a stable plasmonic activity and consequently a high field enhancement. It was observed during our experiments that an efficient near-field enhancement is achieved for a gold coating thickness of approximately $13 \mathrm{~nm}$. Yang et al. reported on a three-dimensional finite-difference time domain (3D-FDTD) simulation to investigate the field-enhancement of a gold tip [22]. The simulated data showed that the TERS enhancement was increased with increasing the tip radius from 15 to $50 \mathrm{~nm}$ after which it leveled off. This effect was attributed to the size-dependent nature of the localized surface plasmon resonance. However, tips with a large radius are not desirable for SNEM imaging 
due to their low spatial resolution. The same authors stated that the use of very sharp tips (radii $5 \mathrm{~nm}$ ) effected a strong increase in field enhancement due to the lightning rod effect.

The scattering response of a gold and silicon tip versus the dielectric constants of polymers in the visible region is calculated by point dipole model (Fig. 5.2) [13, 23]. In this model, the scattering probe is assumed to be a polarizable sphere with a radius of $a<<\lambda$ and a dielectric constant of $\varepsilon_{t}$. The detailed description of the model is given in Chapter 4. Fig. 5.2 shows the calculated amplitude as a function of the dielectric constant for a gold and a silicon tip with radii of $12 \mathrm{~nm}$, the dielectric constants, $\varepsilon_{A u}$ and $\varepsilon_{S i}$ used are $-10+2 i$ and $15.07+0.147 i$, respectively [13, 24]. From Fig. 5.2 it is apparent that the gold tip would be expected to scatter larger in amplitude compared to the silicon tip for a range of dielectric constants typical for polymers in the visible region. The slopes of the scattering amplitude of the PS $\left(\varepsilon_{P S}=2.5\right)$ and P2VP $\left(\varepsilon_{P 2 V P}=2.4\right)$ showed a factor 2 increase in optical contrast for the gold coated tip compared to a bare silicon tip. This is in agreement with the results shown in Fig. 5.1. However, a complete quantitative comparison between the experimental data and the model calculations is not possible for several reasons.

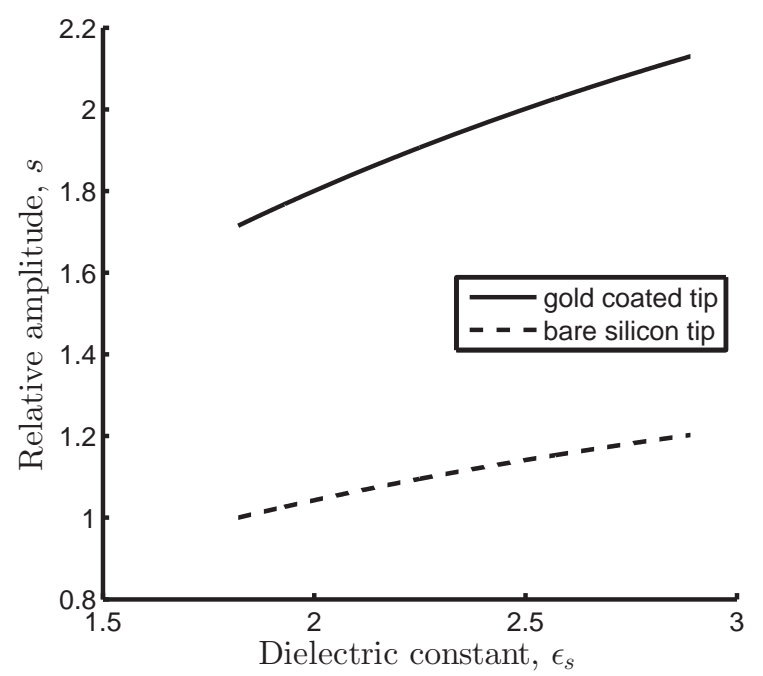

Figure 5.2: Optical near-field amplitude versus the dielectric constant of polymers in the visible region calculated for gold and bare silicon AFM tips by using the point dipole model. The amplitude change is calculated for a tip radius of $12 \mathrm{~nm}$ and for tip-sample contact.

Firstly, the model calculations shown in Fig. 5.2 represent relative changes in scattered amplitude for the tip-sample system which, while clearly modifying the resultant polarization state at the analyzer, do not translate directly into the changes in polarization state relative to the initial null condition detected experimentally. 
Secondly, the model calculations assumed an identical radius for gold and silicon AFM tips, to exclusively investigate the effect of the tip material on the scattering amplitude. However, the gold coated tip used in our experiments has a larger tip radius compared to the bare silicon tip which would be expected to affect the observed intensity of the optical signal.

Finally, it should be noted that the simple point dipole model used does not account for field enhancement effects due to localized surface plasmon modes associated with more realistic non-spherical tip profiles or due to the "lightningrod effect" [25]. Nevertheless, while only a qualitative comparison can be made between the experimental results and the model calculations, both indicate that the gold coating results in a stronger local scattering effect at the tip and therefore greater contrast in the SNEM image.

\subsubsection{Staining of the polymer film}

To further investigate the role of dielectric constant variation in the SNEM contrast mechanism, the microphase separated PS-b-P2VP diblock copolymer film was exposed to iodine vapor to selectively stain the P2VP domains and thus increased the dielectric constant difference between the domains [26]. This procedure has been used successfully in transmission electron microscopy (TEM) studies for PS- $b$-P2VP films [19]. Fig. 5.3 shows the height and optical images of the PS- $b$-P2VP diblock copolymer film after and before exposure to iodine vapor.

An increase in the amplitude of the intensity variations of the optical images following exposure to the iodine vapor was observed, while the height signal was only slightly changed. The amplitude of the intensity variations for the SNEM images were determined by the RMS deviation from the mean value and found as 0.3 and $2.7 \mathrm{mV}$ for images captured before and after the iodine exposure, respectively. This significant change was attributed to presence of the iodine only on the P2VP domains and it thus demonstrates the high sensitivity of SNEM to the differences in the dielectric constant of the sample.

For better direct comparison, the images in Fig. 5.3 were set to the same $z$ scale. Due to the much lower average intensity of the SNEM image before the iodine staining, the image in Fig. 5.3(b) did not clearly show the contrast between the block copolymer domains. Therefore, the same image is displayed again in Fig. 5.4(a) with different $z$-scale to clarify that the optical contrast between the domains of the block copolymer is present before the staining as well. 
(a)

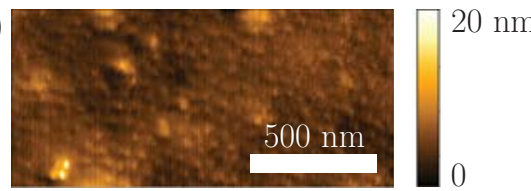

(c)

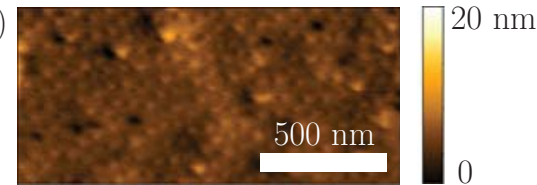

(e)

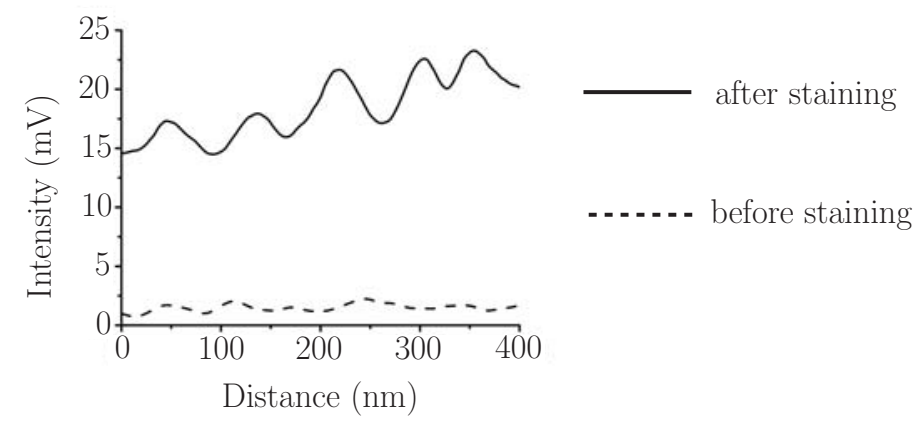

(b)

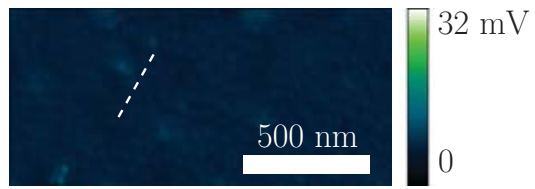

(d)

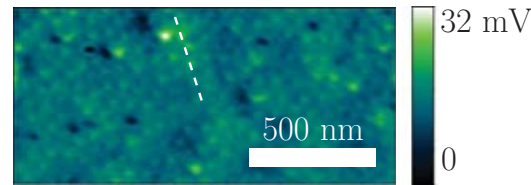

Figure 5.3: SNEM data collected with a gold coated AFM tip: (a) AFM height image and (b) corresponding optical image of a PS- $b$-P2VP film before exposure to iodine vapor. (c) AFM height image and (d) corresponding optical image of PS-b-P2VP film after exposure to iodine vapor. In (e), the corresponding cross-sections along the white dashed lines are shown for the optical ((b) and (d)) images. Images were collected at $2 \mu \mathrm{m} \mathrm{s}^{-1}$ with $512 \times 341$ pixels resolution.

\subsubsection{NAP images and model calculations}

Optical images of the block copolymer film were recorded by utilizing the so called NAP mode at three different tip sample separations $\Delta z=0,10$ and $50 \mathrm{~nm}$. In the NAP mode a line was scanned first in contact with the sample followed by scanning the same line with a height offset (termed NAP height) following the same $z$ profile of the first scan line [27]. Hence the tip-sample distance was kept at a constant distance for each scanned point.

Fig. 5.5 shows the obtained optical NAP images of the PS- $b$-P2VP diblock copolymer film. At $50 \mathrm{~nm}$ tip sample separation, the amplitude of the intensity variations was reduced while for a NAP height of $10 \mathrm{~nm}$ the amplitude of the intensity variations was nearly identical to the image obtained under scanning at intermittent contact (i.e. NAP height is 0 ). The amplitudes of the intensity variations, RMS deviation from the mean, were $0.618,0.616$ and $0.451 \mathrm{mV}$ for the optical images collected at $\Delta z=0,10$ and $50 \mathrm{~nm}$ tip-sample separations, respectively. 
(a)

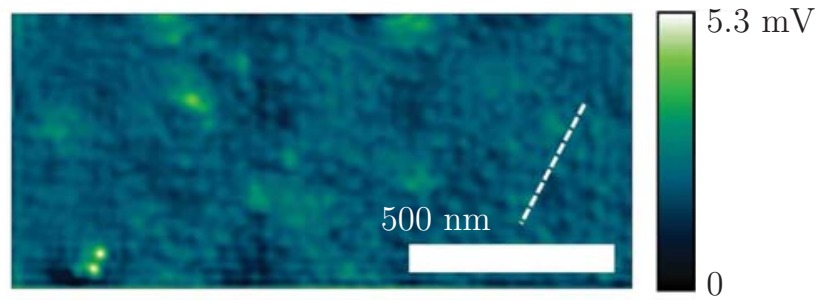

(b)

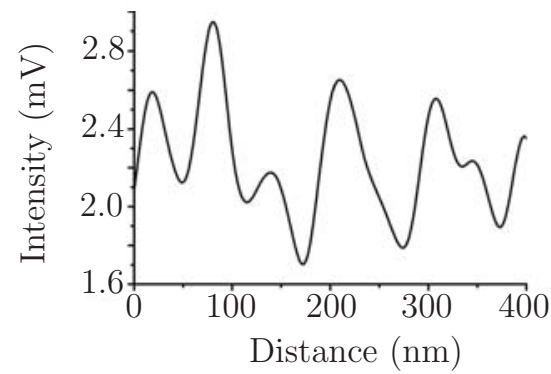

Figure 5.4: SNEM data collected with a gold coated AFM tip: (a) optical image of a PS- $b$-P2VP film before exposure to iodine vapor, (b) the corresponding cross-section along the white dashed line shown in the optical image. The image in Fig. 5.3 is reproduced here with different $z$-scale to reveal the optical contrast between the block copolymer domains.

Note that the amplitudes of the intensity variations were calculated over an area of $600 \times 600 \mathrm{~nm}^{2}$ for all the optical images to elliminate the contribution of the structures revealing an average height of around $10 \mathrm{~nm}$. These structures appear to be larger than the diblock copolymer domains and are assigned to defects which do not change the overall conclusion of the study.

The scattering optical contrast was calculated by the slope of the amplitude of the effective polarizability for PS and P2VP at various tip-sample distances using point dipole model (Fig. 5.6). The typical non-linear decay of the scattering amplitude was observed in Fig. 5.6 [13]. As previously noted, a full quantitative comparison of such calculations to the experimental data is not possible. However, the relative reduction in scattered amplitude as the tip-sample separation increases is consistent with the decrease in the amplitudes of the intensity variations of the SNEM optical images shown in Fig. 5.5. This confirms the role of the light scattered from the tip-sample region in the SNEM contrast mechanism. 
(a)
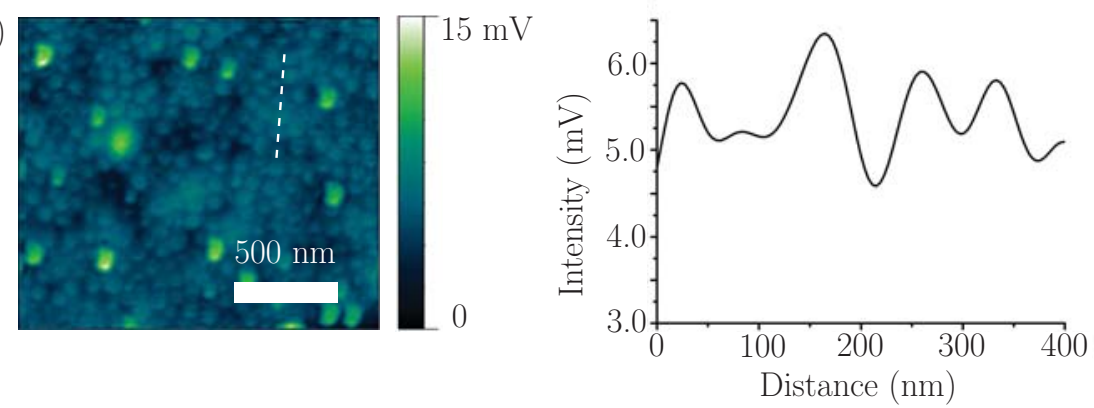

(b)
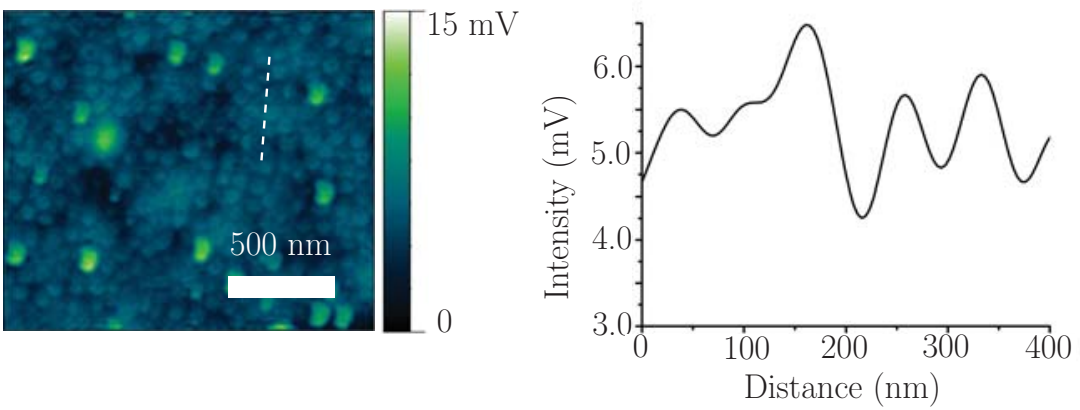

(c)
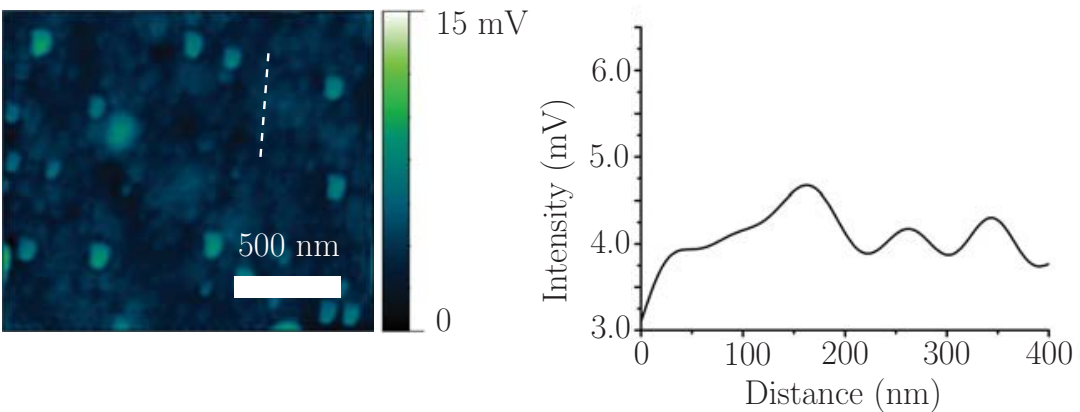

Figure 5.5: SNEM optical images and corresponding cross-sections of a PS- $b$-P2VP diblock copolymer film collected at a lift off distance of (a) 0 , (b) 10 and (c) $50 \mathrm{~nm}$. Images were collected at $2 \mu \mathrm{m} \mathrm{s}^{-1}$ with $512 \times 512$ pixels resolution. 


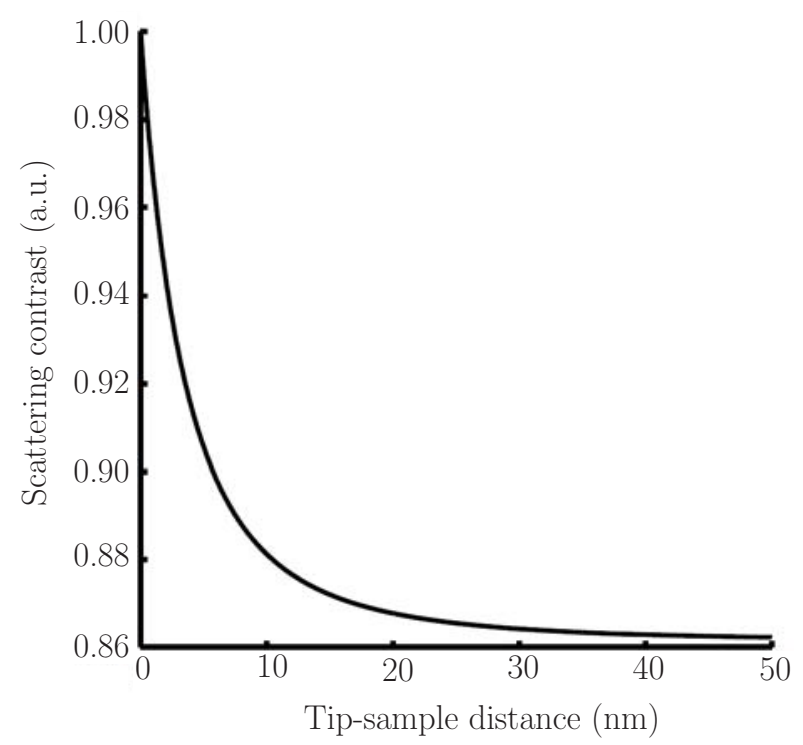

Figure 5.6: Calculated scattering optical contrast between PS and P2VP is plotted versus the distance between the tip and the sample. The scattering contrast amplitude is normalized to the tip-sample contact. The amplitude is calculated for a tip radius of $a=12 \mathrm{~nm}$ and dielectric constant of $\varepsilon_{t}=-10+2 i$.

\subsection{Conclusions}

SNEM imaging of the microphase separated nanoscale morphology of a PS- $b$-P2VP diblock copolymer film was carried out to improve understanding of the optical contrast mechanism. The effect of gold coating of the AFM tip on the optical signal was studied by comparing the optical images captured with a gold coated and a bare silicon tip. The optical image collected with the gold coated AFM tip showed high amplitude of the intensity variations between the blocks of the diblock copolymer having different dielectric constants. However, in images collected using the bare silicon tip the amplitude of the intensity variations was low. This was attributed to the increased polarizability of the gold-coated tip compared to uncoated silicon and stronger local field enhancement in the case of the gold tip due to both localized surface plasmon resonances and the geometric "lightening rod" effect.

Calculations of the scattering amplitude for gold and silicon tips based on the point dipole model supported the experimental results. To further demonstrate that the intensity variations in the optical image are mainly due to the dielectric constant difference in the sample, one of the blocks of the diblock copolymer was selectively stained with iodine to increase the dielectric constant difference. The optical image of the iodine stained block copolymer showed higher ampli- 
tude of the intensity variations, calculated by the RMS deviation from the mean, compared to the non-stained sample.

Finally, the optical images recorded at tip-sample separation distances of 0,10 and $50 \mathrm{~nm}$ were compared. The amplitude of the intensity variations was significantly reduced for the image collected at the $50 \mathrm{~nm}$ tip-sample distance. This result was consistent with the calculated decay of the scattered near-field contrast amplitude of the PS and P2VP domains as the probe was withdrawn from the sample surface. This provided further evidence for the role played by localized tip-sample scattering in SNEM imaging.

\subsection{Experimental section}

Materials: PS- $b$-P2VP diblock copolymer with a number average molar mass, $\mathrm{M}_{n}$, of 103000 and $40500 \mathrm{~g} \mathrm{~mol}^{-1}$ for the PS and P2VP block, respectively and a polydispersity index of 1.09 was purchased from Polymer Source Inc. (Montreal, Canada). Resublimed iodine was provided from Merck (Germany). Chloroform was obtained from Biosolve (Valkenswaard, The Netherlands). Hydrogen peroxide $\left(\mathrm{H}_{2} \mathrm{O}_{2}\right) 30 \%$ was purchased from Merck (Darmstadt, Germany) and sulfuric acid $\left(\mathrm{H}_{2} \mathrm{SO}_{4}\right)$ 95-97\% was bought from Sigma-Aldrich (St. Louis, MO, USA). All materials were used as received. Milli-Q water was produced by a Milipore Synergy system (Billereca, MA, USA). Schott Borofloat glass slides (1 mm thick) were purchased from Sydor Optics (Rochester, NY, USA).

Sample preparation: The PS- $b$-P2VP diblock copolymer was dissolved in chloroform (0.5 wt.\% solution) and spin coated on a glass slide at $1000 \mathrm{rpm}$ for $1 \mathrm{~min}$ to achieve a film thickness of approximately $65 \mathrm{~nm}$. The film thickness was determined by AFM. Prior to spin coating the glass slide was cleaned with piranha solution (solution of $1: 3(\mathrm{v} / \mathrm{v}) 30 \% \mathrm{H}_{2} \mathrm{O}_{2}$ and concentrated $\mathrm{H}_{2} \mathrm{SO}_{4}$ ) and rinsed with copious amounts of high-purity water (Millipore Milli-Q water). Caution: Piranha solution is highly corrosive. Extreme care should be taken when handling Piranha solution and only small quantities should be prepared. The spin coated polymer film was solvent annealed by exposing it to chloroform vapor at room temperature for $16 \mathrm{~h}$. Subsequently the sample was dried under vacuum for $2 \mathrm{~h}$ with a Leybold trivac rotary vane pump at room temperature [28]. To selectively stain the P2VP domains the PS- $b$-P2VP diblock copolymer film was exposed to iodine vapor for $2 \mathrm{~h}$. Subsequently, the film was left in air for 1 day at room temperature in order to remove any unbound iodine.

SNEM: SNEM measurements were carried out in tapping mode AFM imaging using silicon cantilevers (PointProbe Plus silicon probes, PPP-NCH, Nanosensors, Neuchatel, Switzerland) with a nominal resonance frequency and a spring constant of approximately $330 \mathrm{kHz}$ and approximately $42 \mathrm{Nm}^{-1}$, respectively. Probes were investigated both as supplied (bare silicon) and with a $13 \mathrm{~nm}$ sputtered gold coating using a Balzers SCD 040 sputtering machine at an argon pressure of 
0.05 mbar (250 sec sputtering at a $15 \mathrm{~mA}$ current). Images were collected in air at scan rates of 400 and $2000 \mathrm{~nm} \mathrm{~s}^{-1}$ with $512 \times 512$ and $1024 \times 1024$ pixels resolutions. The tip vibrated perpendicularly to the sample surface with an amplitude of approximately $32 \mathrm{~nm}$ (i.e. $64 \mathrm{~nm}$ peak to peak). The tip oscillation amplitude of similar range was used previously for s-SNOM [29, 30]. The amplitude set point was maintained by a feedback loop at approximately $76-80 \%$ of the free oscillation amplitude. The SNEM optical images were processed by polynomial background leveling, followed by two-dimensional fast Fourier transform (2D-FFT) filtering, to remove unwanted periodic structures attributed to electronic noise at fixed frequencies using Gwyddion software [31]. 


\section{Bibliography}

[1] Azoulay, J.; Débarre, A.; Richard, A.; Tchénio, P. J. Microsc. Oxford 1999, $194,486-490$.

[2] Bailo, E.; Deckert, V. Chem. Soc. Rev. 2008, 37, 921-930.

[3] Ichimura, T.; Hayazawa, N.; Hashimoto, M.; Inouye, Y.; Kawata, S. Phys. Rev. Lett. 2004, 92, 220801 1-4.

[4] Karageorgiev, P.; Orendi, H.; Stiller, B.; Brehmer, L. Appl. Phys. Lett. 2001, 79, 1730-1732.

[5] Asghari-Khiavi, M.; Wood, B. R.; Hojati-Talemi, P.; Downes, A.; McNaughton, D.; Mechler, A. J. Raman Spectrosc. 2012, 43, 173-180.

[6] Wang, J.; Wu, X.; Wang, R.; Zhang, M. Electronic Properties of Carbon Nanotubes 2011, 211-264.

[7] Schmid, T.; Opilik, L.; Blum, C.; Zenobi, R. Angew. Chem. Int. Edit. 2013, 52, 5940-5954.

[8] Zayats, A. V. Opt. Commun. 1999, 161, 156-162.

[9] Behr, N.; Raschke, M. B. J. Phys. Chem. C 2008, 112, 3766-3773.

[10] Novotny, L.; van Hulst, N. Nat. Photonics 2011, 5, 83-90.

[11] Hecht, B.; Sick, B.; Wild, U. P.; Deckert, V.; Zenobi, R.; Martin, O. J. F.; Pohl, D. W. J. Chem. Phys. 2000, 112, 7761-7774.

[12] Cumurcu, A.; Duvigneau, J.; Lindsay, I. D.; Schön, P. M.; Vancso, G. J. Eur. Polym. J. 2013, 49, 1935-1942.

[13] Keilmann, F.; Hillenbrand, R. Phil. Trans. R. Soc. Lond. A 2004, 362, 787805 .

[14] Tranchida, D.; Diaz, J.; Schön, P.; Schönherr, H.; Vancso, G. J. Nanoscale 2011, 3, 233-239.

[15] Cumurcu, A.; Duvigneau, J.; Lindsay, I. D.; Schön, P. M.; Vancso, G. J. MRS Online Proceedings Library 2013, 1562.

[16] Leibler, L. Macromolecules 1980, 13, 1602-1617.

[17] Bates, F. S.; Fredrickson, G. H. Phys. Today 1999, 52, 32-38.

[18] Abetz, V.; Simon, P. F. W. Adv. Polym. Sci. 2005, 1984, 125-212.

[19] Roland, S.; Gaspard, D.; Prud'homme, R. E.; Bazuin, C. G. Macromolecules 2012, 54, 5463-5476. 
[20] Methani, D.; Lee, N.; Hartschuh, R. D.; Kisliuk, A.; Foster, M. D.; Sokolov, A. P.; Caiko, F.; Tsukerman, I. J. Opt. A: Pure Appl. Opt. 2006, 8, 183-190.

[21] Deckert-Gaudig, T.; Deckert, V. Phys. Chem. Chem. Phys. 2010, 12, 1204012049 .

[22] Yang, Z.; Aizpurua, J.; Xu, H. J. Raman Spectrosc. 2009, 40, 1343-1348.

[23] Knoll, B.; Keilmann, F. Opt. Commun. 2000, 182, 321-328.

[24] Aspnes, D. E.; Theeten, J. B. J. Electrochem. Soc. 1980, 127, 1359-1365.

[25] Hillenbrand, R.; Taubner, T.; Keilmann, F. Nature 2002, 418, 159-162.

[26] Jang, S. G.; Khan, A.; Hawker, C. J.; Kramer, E. J. Macromolecules 2012, 45, 1553-1561.

[27] Fragouli, D.; Torre, B.; Bertoni, G.; Buonsanti, R.; Cingolani, R.; Athanassiou, A. Microsc. Res. Techniq. 2010, 73, 952-958.

[28] Maas, J. H.; Stuart, M. A. C.; Fleer, G. J. Thin Solid Films 2000, 358, 234-240.

[29] Bachelot, R.; Gleyzes, P.; Boccara, A. C. Opt. Lett. 1995, 20, 1924-1926.

[30] David, T.; Chicanne, C.; Richard, N.; Krenn, J. R.; Scheurer, F.; Ounadjela, K.; Hehn, M.; Lacroute, Y.; Goudonnet, J. P. Rev. Sci. Instrum. 1999, 70, 4587-4594.

[31] Necas, D.; Klapetek, P. Cent. Eur. J. Phys. 2012, 10, 181-188. 


\section{Chapter 6}

\section{SAM modification of gold coated AFM probes}

Tip-enhanced nanoscale optical imaging techniques such as apertureless scanning near-field optical microscopy (a-SNOM) and scanning near-field ellipsometric microscopy (SNEM) applications can suffer from a steady degradation in performance due to adhesion of atmospheric contaminants to the metal coated tip. In this chapter, it is demonstrated that a self-assembled monolayer (SAM) of ethanethiol (EtSH) is an effective means of protecting gold-coated atomic force microscopy (AFM) probe tips from accumulation of surface contaminants during prolonged exposure to ambient air. The period over which they yield consistent and reproducible results for SNEM imaging is thus extended. SNEM optical images of a microphase separated polystyrene-block-poly (methylmethacrylate) (PS-b-PMMA) diblock copolymer film, which were captured with bare and SAMprotected gold-coated AFM probes, both immediately after coating and following five days of storage in ambient air, were compared. During this period the intensity of the optical signals from the untreated gold tip fell by $66 \%$, while those from the SAM protected tip fell by $14 \%$. Additionally, gold coated AFM probe tips were modified with various lengths of alkanethiols to measure the change in intensity variations in the optical images with SAM layer thickness. The experimental results were compared with the point dipole model calculations. 
Parts of this chapter is submitted to:

Cumurcu, A., Diaz, J., Lindsay, I. D., de Beer, S., Duvigneau, J., Schön, P. M. and Vancso, G. J., Optical imaging beyond the diffraction limit by SNEM: Effects of AFM tip modifications with thiol monolayers on imaging quality, Ultramicroscopy. 


\subsection{Introduction}

Unstable plasmonic activity of a probe tip can originate from multiple sources including mechanical [1], thermal [2] and chemical degradation [3]. Studies on silver coated tips used in tip enhanced Raman scattering (TERS) showed that exposure to ambient air resulted in a steady reduction in the tip-enhanced signal over time scales of several days, a behaviour attributed to sulfidation of the silver surface via reaction with sulfur-containing compounds in the atmosphere [4-6]. In addition, it was reported that under high humidity conditions the chemical degradation rate of the silver coated tips was increased $[5,6]$. While acknowledging the lower reactivity of gold as opposed to silver in this context, another TERS study observed degradation of the enhancement by gold coated tips over similar timescales, attributing this to contamination both from the samples examined and atmospheric species [7]. A study of spectral fluctuations in TERS experiments with gold probes showed that such fluctuations could be induced by adsorbing carbon-based species onto the tip and noted such adsorption could also occur from ambient air [8].

Attempts at stabilizing the plasmonic activity of metallic tips against contamination have mainly focused on covering the metal surface with protective coatings. Aluminum oxide $\left(\mathrm{Al}_{2} \mathrm{O}_{3}\right)$ or silicon oxide $\left(\mathrm{SiO}_{x}\right)$ have been shown to preserve the stability of the silver coated tips stored in air up to 40 days [4, 5]. However, the $3 \mathrm{~nm}$ thick protective coating has shown to decrease the signal enhancement approximately 10 to $20 \%$. To our knowledge, the only study on mitigating the effects of contaminants on the plasmonic activity of gold coated tips proposed removal of the contaminations by cleaning the tips in concentrated sulfuric acid [7]. By this method organic adsorbents can be removed but the contamination process cannot be prevented. Furthermore, it is difficult to handle tips in strong acidic solutions and there is the risk of removing some of the gold layer from the tip surface along with the contaminations. Therefore, developing gold coated probes with a stable plasmonic activity remains a challenge.

An alternative approach to protect silver coated tips utilised a self-assembled monolayer (SAM) for TERS in liquid [9]. Alkanethiols $\left(\mathrm{CH}_{3}\left(\mathrm{CH}_{2}\right)_{n} \mathrm{SH}\right)$ adsorb from solution onto noble metal surfaces and form monolayer films generating hydrophobic surfaces with indefinite stability at room temperature with significantly lower surface energy than a bare gold surface [9-12]. Compared to $\mathrm{Al}_{2} \mathrm{O}_{3}$ and $\mathrm{SiO}_{x}$ coatings with $3 \mathrm{~nm}$ thickness, these layers have the advantage of a minor enhancement decrease due to the thickness of the protection layer, since the layer thickness and the packing density of the monolayers depends on the chain length of the thiols (1.5 $\AA$ per $\mathrm{CH}_{2}$ group) allowing controlled thin layers to be formed [10]. Techniques for thiol-based SAM functionalization of AFM tips are well understood and have been widely reported for purposes such as writing patterns with sub-100 nm dimensions [13-15], studying single chemical bond strength [16], chemical specificity [17] and electron transfer [18]. 
In this chapter, the use of a SAM of ethanethiol (EtSH) is described to protect gold coated AFM probe tips from the accumulation of surface contaminants during prolonged exposure to ambient air. SNEM optical images of a microphase separated polystyrene-block-poly (methylmethacrylate) (PS- $b$-PMMA) diblock copolymer film were captured with bare and SAM-protected gold coated probes both immediately after coating and following five days of storage in ambient air.

Gold coated AFM probe tips were modified with various lengths of alkanethiols, namely 1-butanethiol (BuSH), 1-octanethiol (OcSH) and 1-dodecanethiol (DoSH), to determine the length of alkenethiol at which the block copolymer domains are not visible in the optical image. Calculations using a point-dipole model were compared with the experimental data. The morphologies of the AFM probe tips before and after storage, both with and without SAM coating, were investigated with scanning electron microscopy (SEM). Infrared (IR) spectroscopy was used to gain further insight into the formation of the various SAM films.

\subsection{Results and discussion}

The schematic representations in Fig. 6.1 summarize the experiments provided in this chapter. The upper panels show representations of gold coated AFM probe tips covered with various surface layers being scanned across a surface with varying composition and topography, while the lower panels schematically illustrate the corresponding intensity variation detected by SNEM.

SAM of EtSH was used (Fig. 6.1(b)) to protect a gold coated AFM probe tip from the accumulation of surface contaminations during storage (Fig. 6.1(a)). As illustrated in the scheme, the optical contrast would diminish not only when the surface of the gold coating on the AFM probe tips covered with a contamination layer, but also when it is covered with a long chain alkanethiol SAM due to the high coverage and relative thickness (Fig. 6.1(c)) of the SAM.

\subsubsection{Degradation of a gold coated AFM probe tip}

The lifetime of a gold coated AFM probe tip was studied by SNEM imaging of a PS- $b$-PMMA diblock copolymer film (Fig. 6.2). The images were recorded with a freshly prepared gold coated AFM probe tip (Fig. 6.2 (a) and (b)) and with the same tip after it was kept for 5 days under ambient conditions (Fig. 6.2 (c) and (d)). Imaging conditions (amplitude, setpoint) were kept constant throughout the measurements.

Since the average optical intensities may vary depending on the alignment of the laser light on the detector and the nulling conditions, the optical data is compared by the amplitude of the intensity variation arising from the periodic 
(a)
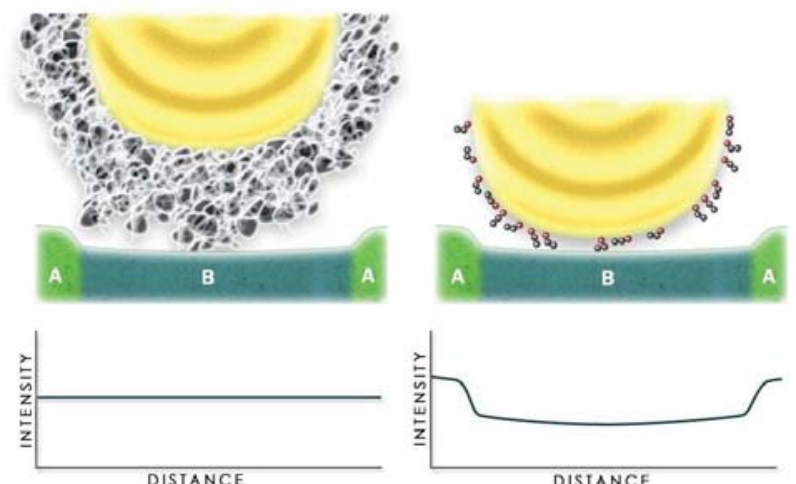

(b)

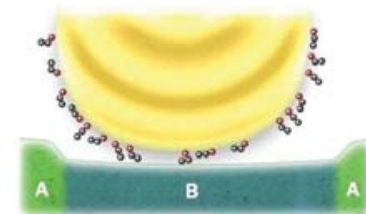

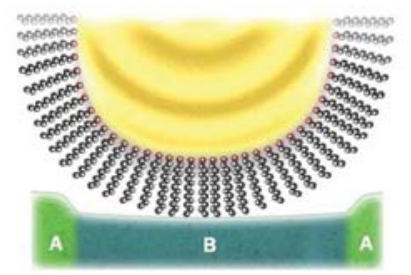

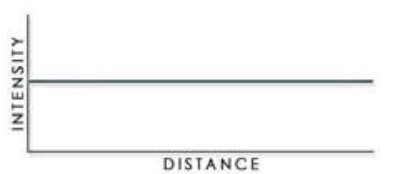

Figure 6.1: Schematic representation of gold coated AFM tips with: (a) contamination layer, (b) short and (c) long chain length SAM, interacting with a microphase-separated block-copolymer film (ABA). The lower diagrams represent schematic optical intensity-distance profiles that would be obtained with the respective applied force in SNEM recordings by scanning across the hypothetical structure shown.

domain pattern formed by the diblock copolymer using the root-mean-square (RMS) deviation from the mean. The RMS optical contrasts, $\mathrm{C}_{R M S}$, are also given calculated using [19]:

$$
C_{R M S}=\sqrt{\frac{1}{N} \sum_{i=1}^{N} \frac{\left(r_{i}-\bar{r}\right)^{2}}{\bar{r}^{2}}},
$$

where $N$ is the number of data points, $\mathrm{r}_{i}$ is the intensity of the $i^{\text {th }}$ data point, and

$$
\bar{r}=\frac{1}{N} \sum_{i=1}^{N} r_{i} .
$$

The amplitudes of the intensity variations and RMS contrasts, $\mathrm{C}_{R M S}$, for the images in Fig. 6.2 (b) and (d), captured with the freshly prepared gold coated AFM probe tip and the same tip after 5 days of storage, were $1.5 \mathrm{mV}\left(\mathrm{C}_{R M S}\right.$ of 0.31$)$ and $0.5 \mathrm{mV}\left(\mathrm{C}_{R M S}\right.$ of 0.24$)$, respectively. This decrease in both the amplitude of the intensity variations and the contrast was ascribed to the accumulation of contaminations from the ambient air and the gel pack during storage [20, 21]. As a consequence the field enhancement by the AFM probe tip at the sample surface, the interaction that forms the basis for the optical contrast in SNEM, was strongly reduced. 
(a)

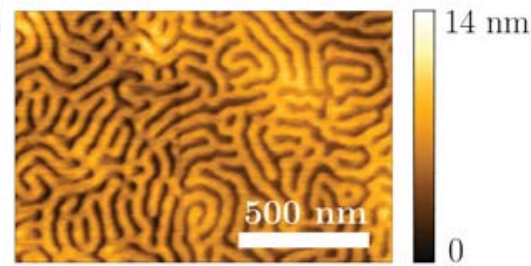

(c)

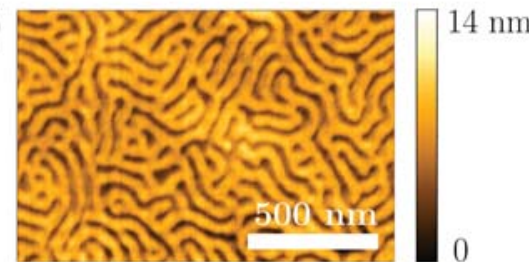

(e) (b)

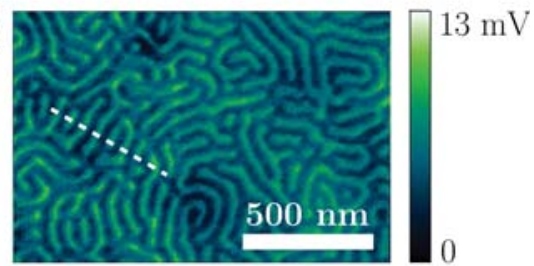

(d)

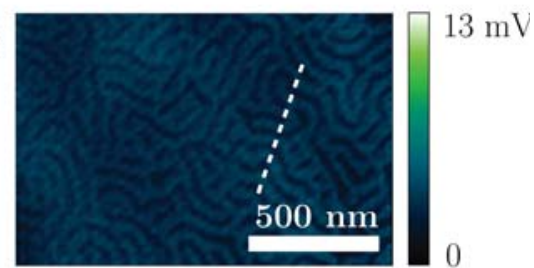

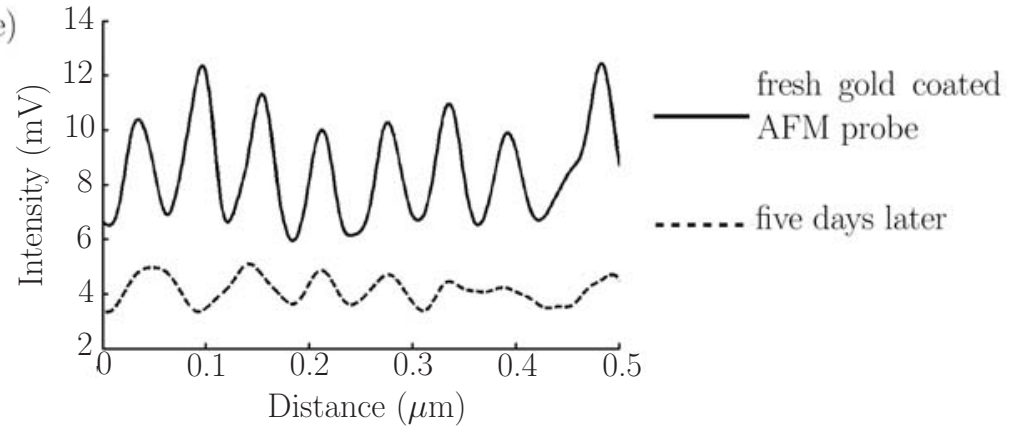

Figure 6.2: SNEM data recorded on a microphase separated PS- $b$-PMMA diblock copolymer film: (a) AFM height, (b) corresponding optical image collected with a freshly prepared gold coated AFM probe. In (c and d), the AFM height and the corresponding optical images collected after five days storage in a closed box in atmospheric air with the same AFM probe tip are shown, respectively. The corresponding cross-sectional optical signals along the white dashed lines (in b and d) are depicted in (e). The images were collected at $0.75 \mu \mathrm{m} \mathrm{s}^{-1}$ with $1024 \times 683 \mathrm{pixel}^{2}$ resolution. The same imaging conditions were used in all the images.

The observed degradation over time was consistent with previously reported results on the accumulation of contaminating species from ambient air on unprotected noble metal TERS probes and SERS active samples [4, 8, 22]. Hence, for tip-enhanced near-field techniques in general, the optical data obtained with a freshly prepared metal coated probe and an aged probe are not directly comparable. 


\subsubsection{Protection of the gold coated AFM probe tips with SAM}

The tapping mode AFM height and the corresponding SNEM optical images of the PS- $b$-PMMA diblock copolymer film which were recorded with an AFM probe tip protected by a SAM of EtSH formed on the surface of the probe immediately following gold coating are shown in Fig. 6.3. Subsequently, as with the untreated tip used to obtain the images shown in Fig. 6.2, the SAM of EtSH protected AFM probe tip was kept in a closed box in atmospheric air for five days.

(a)

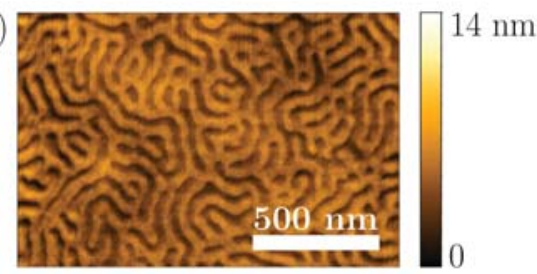

(c)

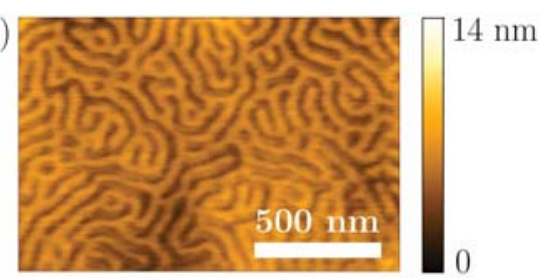

(b)

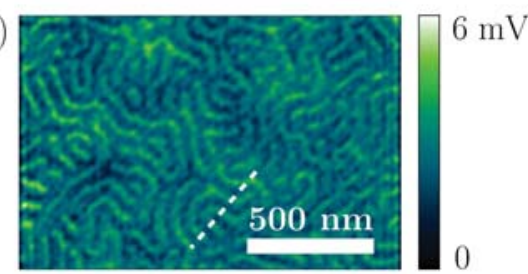

(d)

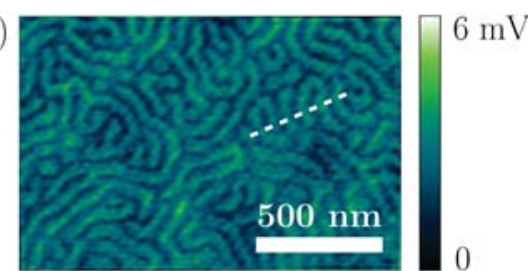

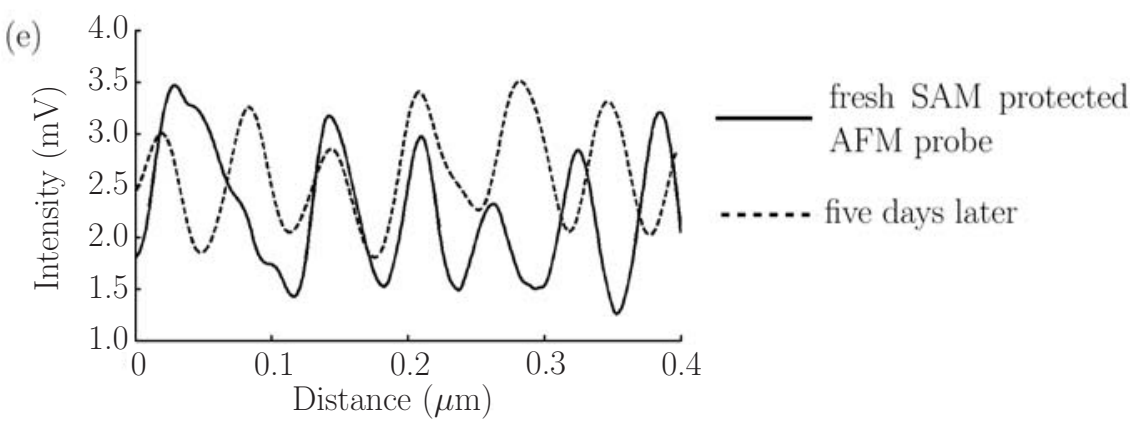

Figure 6.3: SNEM data recorded on a microphase separated PS- $b$-PMMA diblock copolymer film: (a) AFM height and (b) corresponding optical image collected with a fresh SAM of EtSH protected AFM probe tip. In (c and d), the AFM height and the corresponding optical images which were collected with the same tip after five days storage are shown, respectively. The corresponding cross-sectional optical signals along the white dashed lines (in b and $\mathrm{d}$ ) are depicted in (e). The images were collected at $0.75 \mu \mathrm{m} \mathrm{s}^{-1}$ with $1024 \times 683$ pixel $^{2}$ resolution. The same imaging conditions were used in all the images. 
Following storage, the tip was used to record the optical image shown in Fig. 6.3(d) which revealed diblock copolymer domains similar to that obtained with the freshly prepared SAM protected AFM probe tip (Fig. 6.3(b)). The amplitude of the intensity variations of the optical images given in Fig. 6.3(b) and (d) were $0.7 \mathrm{mV}\left(\mathrm{C}_{R M S}\right.$ of 0.29$)$ and $0.6 \mathrm{mV}\left(\mathrm{C}_{R M S}\right.$ of 0.27$)$, respectively. The AFM probe tip protected with a SAM of EtSH thus showed a $14 \%$ degradation in RMS intensity variation and 7\% degradation in RMS contrast as opposed to equivalent figures of $66 \%$ and $33 \%$, respectively, for the freshly prepared bare gold coated tip during the five days of storage time.

SEM inspection of the gold coated and the SAM of EtSH protected AFM probe tips revealed that the morphology of the tips did not change during storage (Fig.6.4). This result explains the similar topographic images recorded in all cases (Fig. 6.2(a) and (c) and Fig. 6.3(a) and (c)) and confirms that no bulk contamination during storage occurred on the AFM probe tip surfaces.

(a)

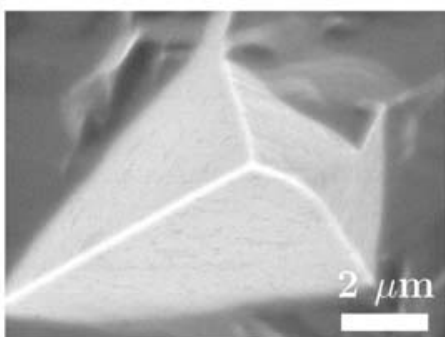

(c)

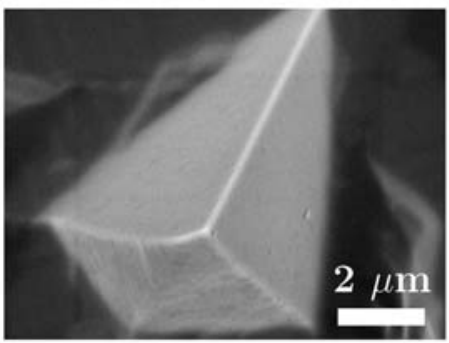

(b)

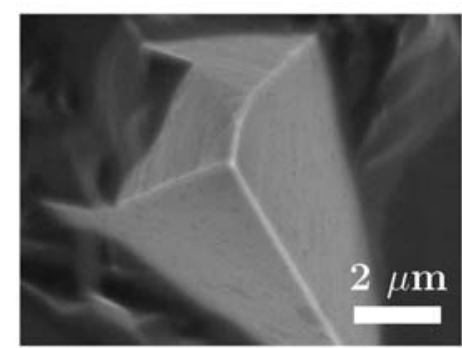

(d)

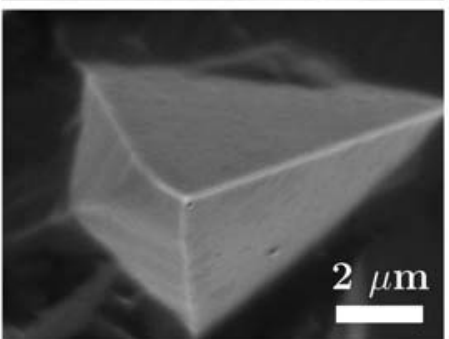

Figure 6.4: SEM images of gold coated silicon AFM tips: (a) freshly gold coated AFM tip, (b) the same gold coated AFM tip after eight days of storage, (c) fresh SAM of EtSH protected AFM tip and (d) the same SAM protected AFM tip after eight days of storage.

It should be noted that, while resulting in greater long-term stability of the tip properties, SAM deposition on the gold coated AFM probe tip causes an initial decrease in the absolute intensity variation, shown in Fig. 6.3(b), compared to a bare gold coated AFM probe tip, shown in Fig. 6.2(b). The initial decrease of the intensity variation was assigned mainly to the decrease in the polarizability and the shift of the plasmon resonance frequency of the gold coated tip by the presence of the dielectric coating [23, 24]. 
In this way, both the SAM and the slow build-up of atmospheric contaminants result in a similar reduction in tip polarizability with the important difference that application of the SAM is controlled and immediately stabilises the tip preventing further degradation. It is unlikely that the tip picking up large scale contamination from the sample was the reason for the optical contrast decease, as the height images in Fig. 6.2 and Fig. 6.3 did not show any signs of tip contamination during sample scanning.

\subsubsection{SAM modification of gold coated tips with various thiols}

Since the optical images captured with the AFM probe tips modified with a SAM of EtSH showed an initial decease in the amplitude of the intensity variation, different lengths of alkanethiols were used to determine the length of the SAM that suppresses the visibility of the block copolymer domains in the optical image. SNEM optical images of the PS-b-PMMA diblock copolymer film recorded with the SAM of BuSH, OcSH and DoSH are shown in Fig. 6.5.

(a)

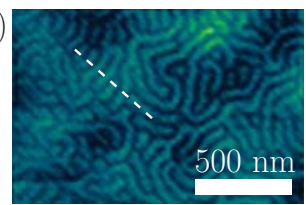

(b)

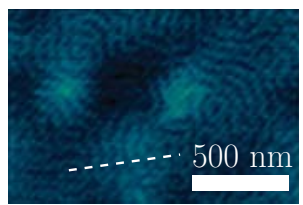

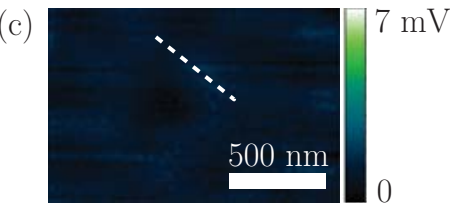

(d)

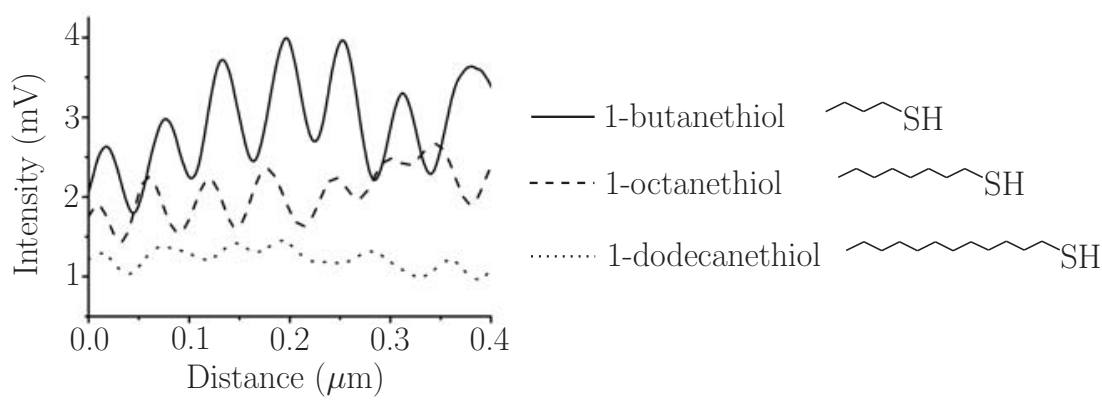

Figure 6.5: SNEM optical images of a microphase separated PS- $b$-PMMA diblock copolymer film recorded with SAM modified AFM tips with various carbon chain lengths: (a) BuSH, (b) OcSH and (c) DoSH. In (d), the cross-sectional optical signal along the corresponding white dashed lines (in a, b and c) are shown. The images were collected at $0.75 \mu \mathrm{m} \mathrm{s}^{-1}$ with $1024 \times 683$ pixel $^{2}$ resolution. The same imaging conditions were used in all the images.

The RMS intensity variations and contrasts of the optical images shown in Fig. 6.5(a) to (c) were $0.60 \mathrm{mV}\left(\mathrm{C}_{R M S}\right.$ of 0.26$), 0.45 \mathrm{mV}\left(\mathrm{C}_{R M S}\right.$ of 0.25$)$ and $0.30 \mathrm{mV}\left(\mathrm{C}_{R M S}\right.$ of 0.25$)$, respectively. The decrease in intensity variations was attributed to in- 
creasing lengths of alkanethiols mainly to the chain length and the packing density of the monolayers since the longer chain alkanethiols $\left(\mathrm{n} \geqslant 9\right.$ for $\left.\mathrm{HS}\left(\mathrm{CH}_{2}\right)_{n} \mathrm{X}\right)$ form ordered and more densely packed structures compared to the shorter chain alkanethiols [10]. Monolayer assemblies of n-alkyl thiols on gold surfaces have previously been characterized by ellipsometry, IR spectroscopy and electrochemistry $[10,11,25,26]$. These studies have shown that long chain thiols form a densely packed crystalline-like assembly with fully extended alkyl chains (average tilt of $20-30^{\circ}$ from the surface normal), while as the chain length decreases, increasingly disordered structures with low coverage and packing density are formed [10].

The point dipole model, which describes the interactions between the polarizable tip and the sample for apertureless SNOM, was used to calculate the scattering amplitude for the tips with dielectric constants corresponding to SAM of BuSH, $\mathrm{OcSH}$ and DoSH $[27,28]$. The scattering amplitude contrasts were calculated from the difference between the scattering amplitudes for the PS and PMMA samples $\left(\varepsilon_{P S}=2.52\right.$ and $\left.\varepsilon_{P M M A}=2.22\right)[29]$. The scattering amplitude contrasts for the AFM probe tips with SAM of BuSH, OcSH and DoSH were found as 15.1, 14.3 and $14.0 \%$ (normalized to the gold scattering amplitude of $100 \%$ ), respectively.

The general trend in the optical contrast decrease of the SAM protected tips compared to the gold coated tip determined by the model calculations agreed with the experimental findings. However, direct comparison between the experimental data and the model calculations are not possible for several reasons. Firstly, the model calculations represent relative changes in scattered amplitude for the tip-sample system which does not directly translate to the polarization changes recorded with SNEM [30]. Secondly, the dielectric constants of the SAM $\left(\varepsilon_{D o S H}=2.10, \varepsilon_{O C S H}=2.13\right.$ and $\left.\varepsilon_{B u S H}=2.20\right)$ was used as the tip dielectric constant in the model calculations [31]. However, in the experiments the underlying gold surface also effects the field enhancement.

The IR spectrum in the $\mathrm{C}-\mathrm{H}$ stretching region is shown for monolayers of BuSH, OcSH and DoSH in Fig. 6.6. Intense peaks at 2919 and $2851 \mathrm{~cm}^{-1}$ (the positions of the $\mathrm{d}^{-1}$ and $\mathrm{d}^{+1} \mathrm{CH}_{2} \mathrm{C}-\mathrm{H}$ stretching modes) in Fig. 6.6(c) indicate the crystallinelike packing of the alkyl chains for the DoSH SAM [26]. However, these peaks do not appear as strongly in the spectra of Fig. 6.6(b) and (a) indicating lower packing of the BuSH and OcSH monolayers. In addition, the bands at 2966 and $2877 \mathrm{~cm}^{-1}$ can be assigned to the $\mathrm{CH}_{3}$ asymmetric in-plane and symmetric $\mathrm{CH}$ stretching mode, respectively. These bands appear in all spectra in the Fig. 6.6 indicating the formation of monolayers in all cases. 


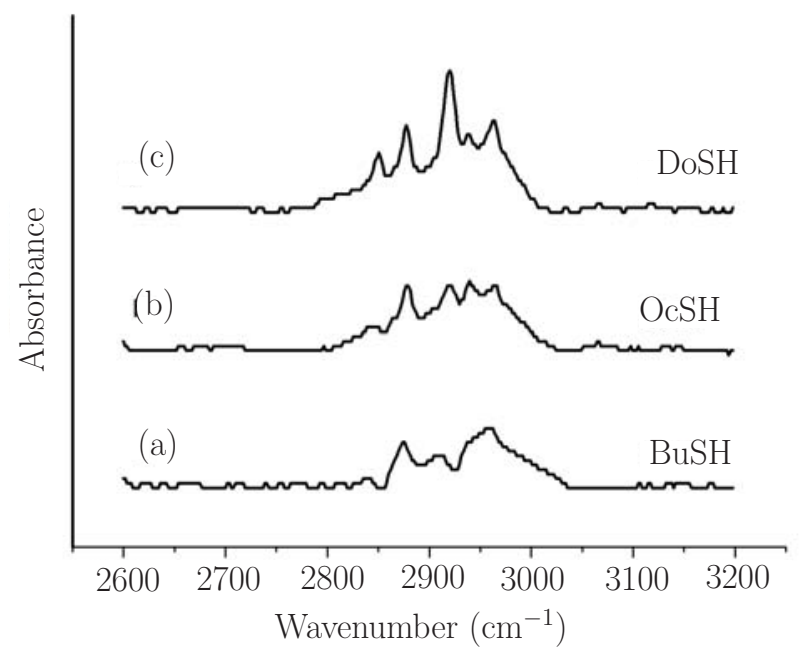

Figure 6.6: IR spectra in the high frequency region for self-assembled n-alkyl thiols on gold surfaces: (a) BuSH, (b) OcSH and (c) DoSH.

\subsection{Conclusions}

The stability of gold coated AFM probe tips for SNEM imaging was improved by protecting the tips with short alkanethiol SAM. SNEM images of a PS-b-PMMA diblock copolymer film, which were recorded with a gold coated AFM probe tip, showed $66 \%$ decrease in the amplitude of the intensity variations, arising from the periodic domain pattern formed by the diblock copolymer, after storing the AFM probe tip five days in ambient air. However, similar images, recorded with an AFM probe tip protected by a SAM of EtSH showed only $14 \%$ decrease in the amplitude of the intensity variations over the same time and storage conditions. Additionally, the amplitudes of the intensity variation of the SNEM optical images were found to decrease when increasing lengths of alkanethiols were used to modify gold coated AFM probe tips.

\subsection{Experimental section}

Materials: PS- $b$-PMMA diblock copolymer with a number average molar mass, $\mathrm{M}_{n}$, of 50000 and $47000 \mathrm{~g} \mathrm{~mol}^{-1}$ for the PS and PMMA block, respectively and a polydispersity index of 1.09 was purchased from Polymer Source Inc. (Montreal, Canada). EtSH (99+\%), BuSH (99+\%), OcSH (97\%)and DoSH (98\%) were obtained from Acros Organics (Geel, Belgium). Toluene and ethanol were supplied from Biosolve (Valkenswaard, The Netherlands). Hydrogen peroxide $\left(\mathrm{H}_{2} \mathrm{O}_{2}\right) 30 \%$ 
was obtained from Merck (Darmstadt, Germany). Sulfuric acid $\left(\mathrm{H}_{2} \mathrm{SO}_{4}\right)$ 95-97\% was bought from Sigma-Aldrich (St. Louis, MO, USA). All materials were used as received. Milli-Q water was produced by a Milipore Synergy system (Billereca, MA, USA). The glass slides were purchased from Thermo Scientific (MenzelGlaser, Gerhard Menzel GmbH, Braunschweig, Germany). The glass slides were made of extra-white soda-lime glass. RMS roughness of a glass slides was 0.77 $\mathrm{nm}$ for a $1.5 \times 1.5 \mu \mathrm{m}^{2}$ area determined by AFM.

Sample preparation: The PS- $b$-PMMA diblock copolymer was dissolved in toluene (1 wt.\% solution) and spin coated on a glass slide at $2000 \mathrm{rpm}$ for 1 minute. Prior to spin coating the glass slide was cleaned with piranha solution (i.e. a solution of $1: 3(\mathrm{v} / \mathrm{v}) 30 \% \mathrm{H}_{2} \mathrm{O}_{2}$ and concentrated $\left.\mathrm{H}_{2} \mathrm{SO}_{4}\right)$ for 20 minutes and rinsed with copious amounts of high-purity water (Millipore Milli-Q water). Caution: P $i$ ranha solution is highly corrosive. Extreme care should be taken when handling Piranha solution and only small quantities should be prepared. The spin coated polymer film was annealed in air at $175{ }^{\circ} \mathrm{C}$ overnight $[32,33]$. The film thickness was $35 \mathrm{~nm}$, as determined by AFM height measurements.

Preparation and the modification of the gold coated AFM tips: Silicon AFM probes (PointProbe Plus silicon probes, PPP-NCH, Nanosensors, Neuchatel, Switzerland) with an average nominal resonance frequency and spring constant of $\sim 330$ $\mathrm{kHz}$ and $\sim 42 \mathrm{~N} \mathrm{~m}^{-1}$, respectively, were sputtered with a $13 \mathrm{~nm}$ thick layer of gold using a Balzers SCD 040 sputtering machine at an argon pressure of 0.05 mbar (250 sec sputtering at a $15 \mathrm{~mA}$ current). The average diameter of the SAM covered gold coated AFM probe tips was determined to be $24 \mathrm{~nm}$ by SEM images.

Solutions of EtSH, BuSH, OcSH and DoSH were prepared in ethanol (concentration of $10^{-3} \mathrm{M}$ ). The gold coated AFM probes were immersed in the solutions for various time periods ( 5 min for $\mathrm{EtSH}, 10 \mathrm{~min}$ for $\mathrm{BuSH}, 20 \mathrm{~min}$ for $\mathrm{OcSH}$ and 16 hours for DoSH) at room temperature to allow the formation of a SAM on the gold surface $[9,11]$. Subsequently, the probes were rinsed with ethanol and air-dried at room temperature.

SNEM: A MFP-3D AFM (Asylum Research, Santa Barbara, CA, USA) was combined with a Multiskop ellipsometer (Optrel, Sinzing, Germany) in the SNEM setup. Samples were placed on a custom-made interface plate (Optrel, Sinzing, Germany). Images were collected in air at AFM scan rates of $750 \mathrm{~nm} \mathrm{~s}^{-1}$ and $683 \times 1024$ pixel $^{2}$ resolutions with tapping mode free amplitude of approximately $74 \mathrm{~nm}$ (peak to peak distance). The amplitude set point was maintained by a feedback loop at approximately $88 \%$ of the free oscillation amplitude. The SNEM optical images were processed in Gwyddion by polynomial background leveling, followed by two-dimensional fast Fourier transform (2D-FFT) filtering, to remove unwanted periodic structures attributed to electronic noise at fixed frequencies [34]. 
Characterization: SAMs of BuSH, OcSH and DoSH on gold substrates were characterized by a Vertex 70 IR spectrometer (Bruker Optik GmbH, Ettlingen, Germany) equipped with a MCT detector. IR spectra were continuously collected with a spectral resolution of $4 \mathrm{~cm}^{-1}$ over 1024 scans. Background spectra were collected on a gold substrate immediately after it was piranha cleaned for 3-5 minutes, rinsed with copious amounts of MilliQ water and ethanol followed by blow-drying in a nitrogen stream. The surface morphologies of the AFM probe tips were studied by field emission scanning electron microscopy (FE-SEM, JEOL, JSM-6330F) at $5 \mathrm{kV}$ of accelerating voltage. 


\section{Bibliography}

[1] Zhu, L.; Atesang, J.; Dudek, P.; Hecker, M.; Rinderknecht, J.; Ritz, Y.; Geisler, H.; Herr, U.; Greer, R.; Zschech, E. Mater. Sci-Poland 2007, 25, 19-31.

[2] Zhang, W. H.; Yeo, B. S.; Schmid, T.; Zenobi, R. J. Phys. Chem. C 2007, 111, 1733-1738.

[3] Mcmahon, M.; Lopez, R.; Meyer, H. M.; Feldman, L. C.; Haglund, R. F. Appl. Phys. B-Lasers O 2005, 80, 915-921.

[4] Barrios, C. A.; Malkovskiy, A. V.; Kisliuk, A. M.; Sokolov, A. P.; Foster, M. D. J. Phys. Chem. C 2009, 113, 8158-8161.

[5] Barrios, C. A.; Malkovskiy, A. V.; Hartschuh, R. D.; Kisliuk, A. M.; Sokolov, A. P.; Foster, M. D. Proc. SPIE 2008, 6954, 69540C-69540C-12.

[6] Barrios, C. A.; Foster, M. D.; Extending Usable Lifetime of Plasmonic Silver Structures Designed For High Resolution Chemical Imaging and for Chemical and Biological Sensing; Tech. Rep. W911NF-07-1-0470; University of Akron; 2008.

[7] Liu, Z.; Wang, X.; Dai, K.; Jin, S.; Zeng, Z. C.; Zhuang, M. D.; Yang, Z. L.; Wu, D. Y.; Ren, B.; Tian, Z. Q. J. Raman Spectrosc. 2009, 40, 1400-1406.

[8] Chaigneau, M.; Picardi, G.; Ossikovski, R. Surf Sci 2010, 604, 701-705.

[9] Schmid, T.; Yeo, B. S.; Leong, G.; Stadler, J.; Zenobi, R. J. Raman Spectrosc. 2009, 40, 1392-1399.

[10] Porter, M. D.; Bright, T. B.; Allara, D. L.; Chidsey, C. E. D. J. Am. Chem. Soc. 1987, 109, 3559-3568.

[11] Bain, C. D.; Troughton, E. B.; Tao, Y. T.; Evall, J.; Whitesides, R. G., G. M. Nuzzo J. Am. Chem. Soc. 1989, 111, 321-335.

[12] Tao, Z.; Bhushan, B. Tribol. Lett. 2006, 21, 1-16.

[13] Piner, R. D.; Zhu, J.; Xu, F.; Hong, S. H.; Mirkin, C. A. Science 1999, 283, 661-663.

[14] Ivanisevic, A.; Mirkin, C. A. J. Am. Chem. Soc. 2001, 123, 7887-7889.

[15] Ginger, D. S.; Zhang, H.; Mirkin, C. A. Angew. Chem. Int. Ed. 2004, 43, 30-45.

[16] Han, T.; Williams, J. M.; Beebe, T. P. Anal. Chim. Acta 1995, 307, 365-376.

[17] Schönherr, H.; Chechik, V.; Stirling, C. J. M.; Vancso, G. J. J. Am. Chem. Soc. 2000, 122, 3679-3687. 
[18] Wold, D. J.; Frisbie, C. D. J. Am. Chem. Soc. 2001, 123, 5549-5556.

[19] Hecht, E. Optics (4th Edition); Addison Wesley: San Francisco, 2001.

[20] Bonaccurso, E.; Gillies, G. Langmuir 2004, 20, 11824-11827.

[21] Lo, Y. S.; Huefner, N. D.; Chan, W. S.; Dryden, P.; Hagenhoff, B.; Beebe, T. P. Langmuir 1999, 15, 6522-6526.

[22] Jacobson, M. L.; Rowlen, K. L. J. Phys. Chem. B 2006, 110, 19491-19496.

[23] Behr, N.; Raschke, M. B. J. Phys. Chem. C 2008, 112, 3766-3773.

[24] Cui, X.; Erni, D.; Zhang, W.; Zenobi, R. Chem. Phys. Lett. 2008, 453, 262-265.

[25] Bain, C. D.; Evall, J.; Whitesides, G. M. J. Am. Chem. Soc. 1989, 111, 7155-7164.

[26] Nuzzo, R. G.; Dubois, L. H.; Allara, D. L. J. Am. Chem. Soc. 1990, 112, $558-569$.

[27] Keilmann, F. J. Electron Microsc. 2004, 53, 187-192.

[28] Keilmann, F.; Hillenbrand, R. Philos T Roy Soc A 2004, 362, 787-805.

[29] Katritzky, A. R.; Sild, S.; Karelson, M. J. Chem. Inform. Comput. Sci. 1998, 38, 1171-1176.

[30] Cumurcu, A.; Duvigneau, J.; Lindsay, I. D.; Schön, P. M.; Vancso, G. J. Eur. Polym. J. 2013, 49, 1935-1942.

[31] Peterlinz, K. A.; Georgiadis, R. Opt. Commun. 1996, 130, 260-266.

[32] Morkved, T. L.; Jaeger, H. M. Europhys. Lett. 1997, 40, 643-648.

[33] Zhang, X. H.; Berry, B. C.; Yager, K. G.; Kim, S.; Jones, R. L.; Satija, S.; Pickel, D. L.; Douglas, J. F.; Karim, A. Acs Nano 2008, 2, 2331-2341.

[34] Necas, D.; Klapetek, P. Cent. Eur. J. Phys. 2012, 10, 181-188. 


\title{
Chapter 7
}

\section{Optical signal blocking and recovery in SNEM}

\begin{abstract}
A gold coated atomic force microscope (AFM) probe tip was modified with a selfassembled monolayer (SAM) of 1-dodecanethiol (DoSH) for scanning near-field ellipsometric microscopy (SNEM) imaging of a microphase separated polystyrene- $b$ poly(methyl methacrylate) (PS-b-PMMA) diblock copolymer film. During imaging at low pressures the optical contrast was greatly reduced compared to a bare gold coated AFM probe tip as explained by dielectric shielding of the gold coated AFM probe tip via the hydrophobic SAM and the reduction of unwanted background scattering from the tip shaft. While a SAM of DoSH was found to strongly suppress field enhancement, it was observed that it can be locally removed from the tip apex by deforming the molecules under load, restoring SNEM image contrast. Intensity-distance curves obtained with a bare gold coated and SAM covered AFM probe tips were compared to investigate scattering of the tips at the far-field.
\end{abstract}


Parts of this chapter is submitted to:

Cumurcu, A., Diaz, J., Lindsay, I. D., de Beer, S., Duvigneau, J., Schön, P. M., and Vancso, G. J., Optical imaging beyond the diffraction limit by SNEM: Effects of AFM tip modifications with thiol monolayers on imaging quality, Ultramicroscopy. 


\subsection{Introduction}

Scanning near-field ellipsometric microscopy (SNEM), similarly to the other tip enhanced near-field optical microscopy techniques, is based on the strong electromagnetic field enhancement that occurs at the apex of the metal coated probing tip due to collective electronic excitations and localized resonant plasmon excitations in noble metal films or nanoparticles [1-3]. The material and geometric characteristics of the probe tip, such as metal type, roughness, size and shape play a crucial role in the field enhancement [4-7]. Hence, besides being able to reproducibly manufacture the probe tips, maintaining the constant tip surface composition and geometric characteristics during scanning and storage have pivotal importance to ensure consistent field enhancement and reproducible optical contrast at high resolution.

In common with other apertureless scanning near-field optical microscopy (aSNOM) techniques, the collection of the tip-scattered light in SNEM cannot be separated from that arising from the wider illuminated region. The tip-scattered near-field signal is therefore mixed with a strong background signal. Other aSNOM techniques separate the background signal from the local near-field scattering using lock-in detection at the tapping mode frequency $[8,9]$. In SNEM, the background scattering is initially minimized by setting the null conditions (finding polarizer and analyzer settings that minimize the light at the detector). The combined contribution of scatterings from the sample, cantilever and the nearfield scattering at the tip-sample interaction region are minimized by nulling at the start of the measurement. However, during scanning the background signal varies with the motion of the tip normal to the sample surface (on scale $\lambda$ due to interference fringes parallel to the sample surface) introducing a cross-talk between height and optical images. This is termed $z$-motion artifact, which yields features in the optical image that are correlated to the structures in the sample topography, thus making image interpretation ambiguous [10-12]. More detailed information on this topic is provided in Chapter 4.

The SAM of thiol functionalization of AFM tips was previously used in the literature for different purposes. For instance, thiol functionalized AFM tips were used to write patterns with sub-100 nm dimensions on gold or silicon surfaces [13-15]. Thiol functionalized AFM tips were also used to study single chemical bond strength by the force-distance curves of AFM [16]. Surface reactions in-situ with chemical specificity at the nanometer scale were studied using thiol of SAM functionalized AFM tips [17]. Additionally, electron transfer in molecules were studied using thiol functionalized probes by conducting AFM [18].

In this chapter, to gain further insight into the role of the effects of SAM coating on SNEM contrast, imaging of a microphase separated polystyrene- $b$-poly(methyl methacrylate) (PS- $b$-PMMA) diblock copolymer was performed using a 1-dodecan ethiol (DoSH) functionalized tip under a range of contact forces. The SAM of DoSH was found to suppres the field enhancement at the tip end. Then, the SAM 
was removed locally by deforming the molecules under load. The amplitude of the intensity variations of the SNEM images captured at increasing loads showed the deformation of the SAM layer. Additionally, SNEM intensity-distance curves captured with a bare gold coated and a SAM functionalized gold coated AFM tips were compared to investigate the far-field scattering of the tips.

\subsection{Results and discussion}

The schematic representations in Fig. 7.1 summarize the experiments in this chapter. The upper panels show representations of SAM of DoSH functionalized gold coated AFM probe tips being scanned across a surface with varying composition and topography at increasing load, while the lower panels schematically illustrate the corresponding intensity variation detected by SNEM. The gold coated AFM probe tip which was modified with DoSH was found to block the near-field enhancement as illustrated in Fig. 7.1(a). The thiol molecules at the apex of this modified AFM probe tip were then removed by scanning at high load. Thus, the underlying gold was revealed as illustrated in Fig. 7.1(c) resulting in SNEM optical images which reveals the phase separated morphology of the diblock copolymer.

(a)
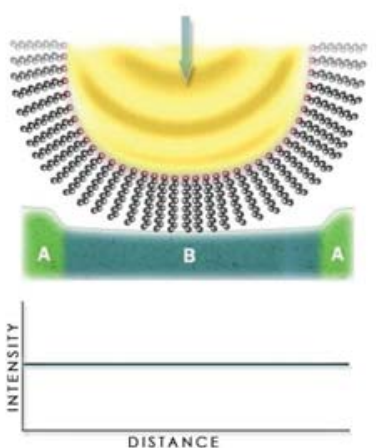

(b)

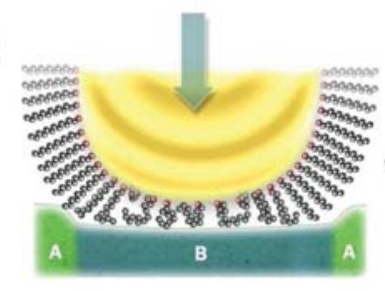

(c)

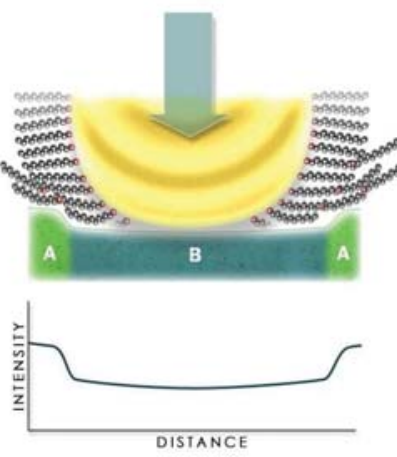

Figure 7.1: Schematic representation of a DoSH functionalized gold coated AFM tip. In ( $\mathrm{a}, \mathrm{b}$ and $\mathrm{c}$ ), the schematic representations of gold coated AFM probe tips modified with a SAM of DoSH illustrating the deformation and the displacement of the SAM at the edge of the AFM tip by increasing load are shown. The load is increased from (a) to (c). The diagrams represent schematic optical intensity-distance profiles that would be obtained with the respective applied force in SNEM recordings by scanning across the hypothetical structure shown. 


\subsubsection{SNEM imaging using a SAM of 1-dodecanethiol func- tionalized tip}

AFM height and SNEM optical images of the PS- $b$-PMMA diblock copolymer thin film captured with a SAM of DoSH modified gold coated AFM probe tip with increasing load are shown in Fig. 7.2. The maximum force and the contact time (Table 7.1) per tapping oscillation were estimated using the VEDA (virtual environment for dynamic AFM) simulation tool with the cantilever parameters described in the experimental section as input for the simulations [19, 20].

Since the adhesion between the SAM modified AFM probe tip and the sample surface is very low, the Hertz contact model was chosen for our tip-sample interactions $[21,22]$. It is important to note that the contact radius can become too large for the Hertz model to be valid. However, due to the relatively large Poisson ratios of the gold and PS, the error by applying the Hertz model is expected to be lower than $10 \%$ [23].

Table 7.1: Applied force, pressure and contact time on each scan

\begin{tabular}{cccc}
\hline \hline $\begin{array}{c}\text { Images in } \\
\text { Fig. 7.2 }\end{array}$ & $\begin{array}{c}\text { Applied force } \\
\mathrm{F}(\mathrm{nN})\end{array}$ & $\begin{array}{c}\text { Pressure } \\
\mathrm{P}(\mathrm{GPa})\end{array}$ & $\begin{array}{c}\text { Contact time } \\
\mathrm{t}(\mu \mathrm{s})\end{array}$ \\
\hline (a) and (b) & 400 & 0.66 & 0.55 \\
(c) and (d) & 600 & 0.76 & 0.62 \\
(e) and (f) & 750 & 0.82 & 0.66
\end{tabular}

The optical image in Fig. 7.2 did not clearly reveal the domains of the block copolymer due to the dense coverage of the SAM of DoSH on the gold surface of the AFM probe tip, except a weak contrast appearing towards the end of the scan indicated with area 3 (scanning direction upwards). Note that the optical images shown in Fig. 7.2 were divided into three sections with white dotted lines and the scan directions are indicated with arrows. The optical images in Fig. 7.2(d) and (f), showed the phase separated morphology of the block copolymer.

From the intensity profiles of Fig. 7.2(g) it can be seen that the data of Fig. 7.2(d) and (f) clearly exhibit the same periodic contrast due to the block copolymer morphology, while the intensity profile cross-section of Fig. 7.2(b) shows little evidence of this periodicity. This result was interpreted as an indication that distortion of the SAM molecules due to the higher pressure applied during the acquisition of Fig. 7.2(d) and (f) resulted in increased exposure of the underlying gold surface and therefore increased tip polarizability.

The SNEM optical images were divided into three areas as shown in Fig. 7.2(b), (d) and (f). The amplitude of the intensity variations from area 1 and 3 are provided, since these areas represent the beginning and the end of a scan. To eliminate the contribution of the background variation, the average amplitude of the intensity variation was calculated from four areas of $200 \times 200 \mathrm{~nm}^{2}$ dimensions that showed constant background signal. The average amplitudes of the intensity 
(a)

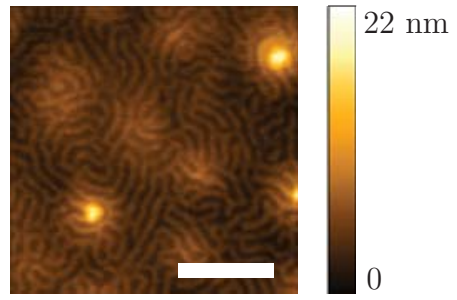

(c)

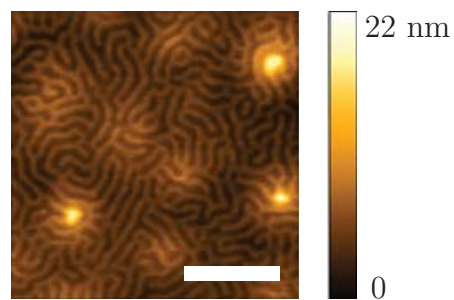

(e)

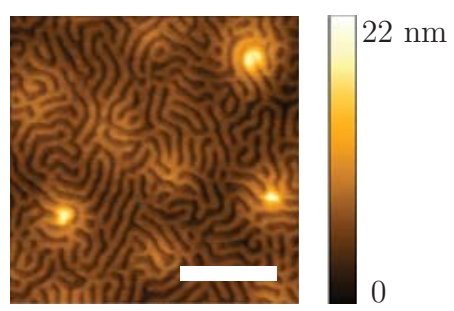

(b)

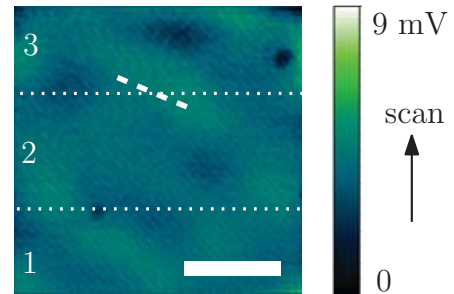

(d)

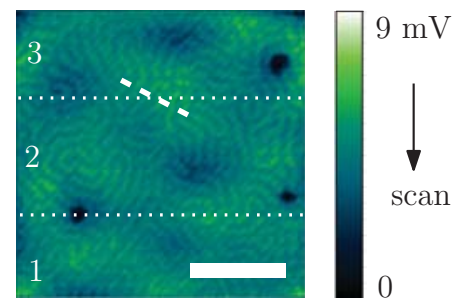

(f)

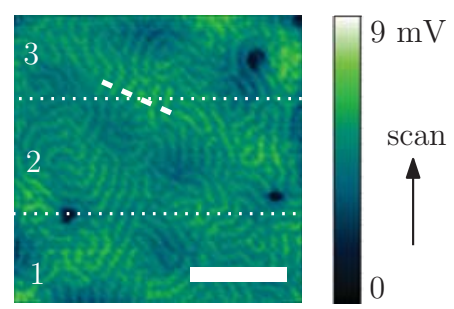

(g)

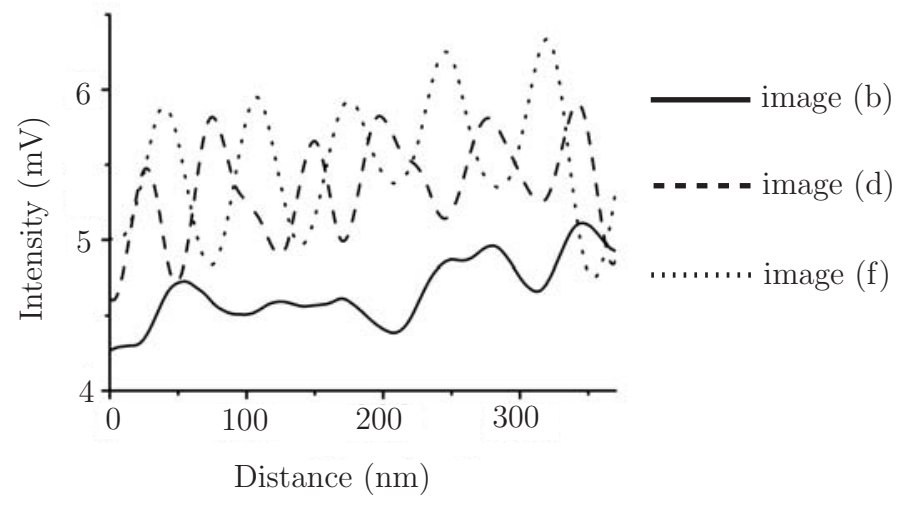

Figure 7.2: A series of AFM height (left) and corresponding SNEM optical (right) images of a microphase separated PS- $b$-PMMA diblock copolymer film. The images are collected at ( $\mathrm{a}$ and b) 0.66, (c and d) 0.76 and (e and f) $0.82 \mathrm{GPa}$ pressures. In (g) the corresponding intensity cross-sections along the white dashed lines for optical images are shown. The scanning direction is shown with arrows. All images are collected at $0.75 \mu \mathrm{m} \mathrm{s}^{-1}$ with $1024 \times 1024$ pixel $^{2}$ resolution. The scale bar represents $500 \mathrm{~nm}$. The SNEM optical images are sectioned into three parts with dotted lines. See the text for details. The set point was kept at $880 \mathrm{mV}$. 
variations (RMS deviation from the mean) within the area 1 were $0.12,0.28$ and $0.45 \mathrm{mV}$ for the Fig. 7.2(b),(d) and (f), respectively. The average amplitudes of the intensity variations (RMS deviation from the mean) within the area 3 were 0.17, 0.32 and $0.4 \mathrm{mV}$ for the Fig. $7.2(\mathrm{~b}),(\mathrm{d})$ and (f), respectively.

The increase in the RMS deviations of both areas with the increase in force was attributed to the local deformation of the thiol molecules at the apex of the AFM probe tip [24-26]. Note that large structures that appeared to be larger than the diblock copolymer domains were excluded when the average amplitude of the intensity variations were calculated. These structures were assigned to defects and they did not change the overall conclusion of the study.

The behaviour of SAM alkanethiols on gold surfaces was previously studied using AFM contact mode imaging under constant normal force using silicon nitride tips with radii of $<100 \mathrm{~nm}$ [27]. Thiol molecules could support a load up to critical values of $\sim 1 \mathrm{GPa}$ at the tip. However, beyond this critical load the thiol molecules showed structures characteristic to the gold substrate in the AFM height images. Upon lowering the pressure, the thiol molecules recovered, returning to the original ordered structure of the SAM in the height image.

In this case, therefore, it is believed that during tapping mode imaging, the thiol molecules at the tip apex were compressed under the load of the tip contacting the sample and recovered when the load was removed on breaking contact. Further, it is suggested that the weak optical contrast shown towards to the end of the image in Fig. 7.2(b) results from the cumulative effect of many compression and release cycles combined with that of lateral forces during scanning leading to the creation of defects in the SAM at the tip and permanent exposure of the underlying gold substrate.

\subsubsection{Gold deformation at the tip apex}

The optical image recorded under 1.3 GPa pressure showed a decrease in the amplitude of the intensity variation due to the permanent plastic deformation of the gold layer $[27,28]$. The average amplitudes of the intensity variations, determined from four areas with dimensions of $200 \times 200 \mathrm{~nm}^{2}$ and constant background variation, of the optical image in Fig. 7.3 for the area 1 and 3 were 0.35 and $0.34 \mathrm{mV}$, respectively. RMS deviation of the both areas found to be lower than the same areas in Fig. 7.2(f). Additionally, a SEM top view image of the used tip is displayed in Fig. 7.4. The SEM image clearly shows the deformation at the tip edge. 
(a)

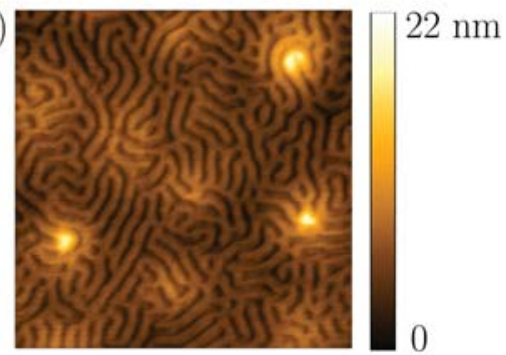

(b)

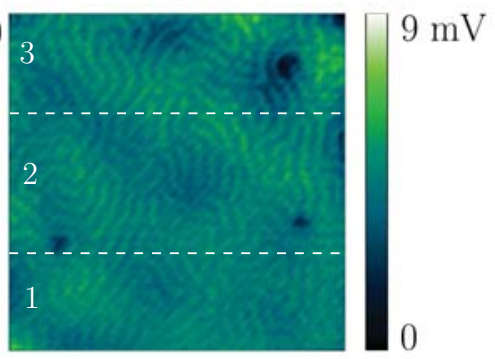

Figure 7.3: SNEM data of a microphase separated PS- $b$-PMMA diblock copolymer film recorded at an applied load of $1.3 \mathrm{GPa}$ to the sample surface: (a) AFM height and (b) optical image.

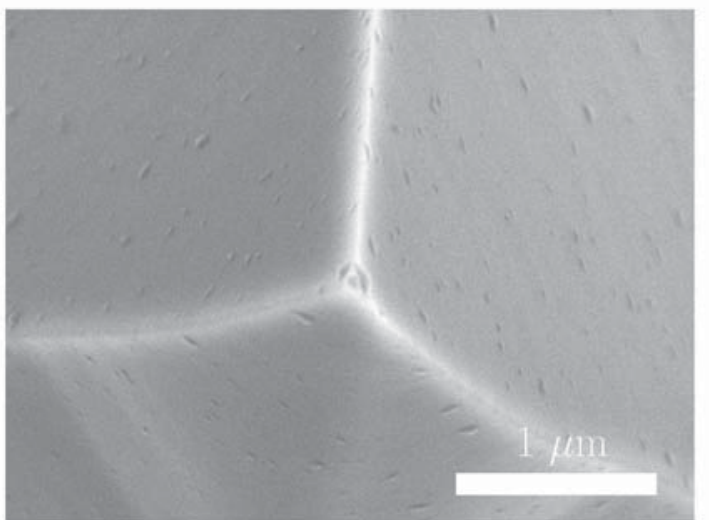

Figure 7.4: SEM image of the SAM covered tip used to image at high pressures $(1.3 \mathrm{GPa})$ above the plastic deformation of the gold layer. 


\subsubsection{SNEM intensity-distance curves}

The intensity recorded as a function of tip-sample distance with a gold coated and a SAM of DoSH covered gold coated AFM probe tip is shown in Fig. 7.5. The tip-sample distance corresponding to zero nm defines the tip and sample hard contact. Both curves regardless of the tip show oscillations originating from the interference of the multiple reflections between the AFM tip including the tip shaft and the sample surface. The AFM tip and the sample act like the two moving mirrors in the Michelson interferometer and show oscillations with a periodicity of $\lambda /(2 \cos \theta)$ equal to $413 \mathrm{~nm}$, where $\lambda$ is the wavelength and $\theta$ is the incident angle of the light $[12,29]$.

The amplitude of the oscillations, however, differ for the gold coated and SAM covered gold coated tips, 0.16 and $0.06 \mathrm{~V}$, respectively. The amplitude difference is attributed to the low reflectivity of the SAM covered AFM probe tip compared to the bare gold coated one. Modifying the gold coated AFM probe tip with the SAM of DoSH minimizes the background signal contribution to the SNEM optical image. It should be also noted that the alignment may also introduce an amplitude change. The intensity-distance curve does not reveal the intensity increase due to the deformation of the SAM of DoSH at the tip edge, since this change is lower than $1 \mathrm{mV}$.

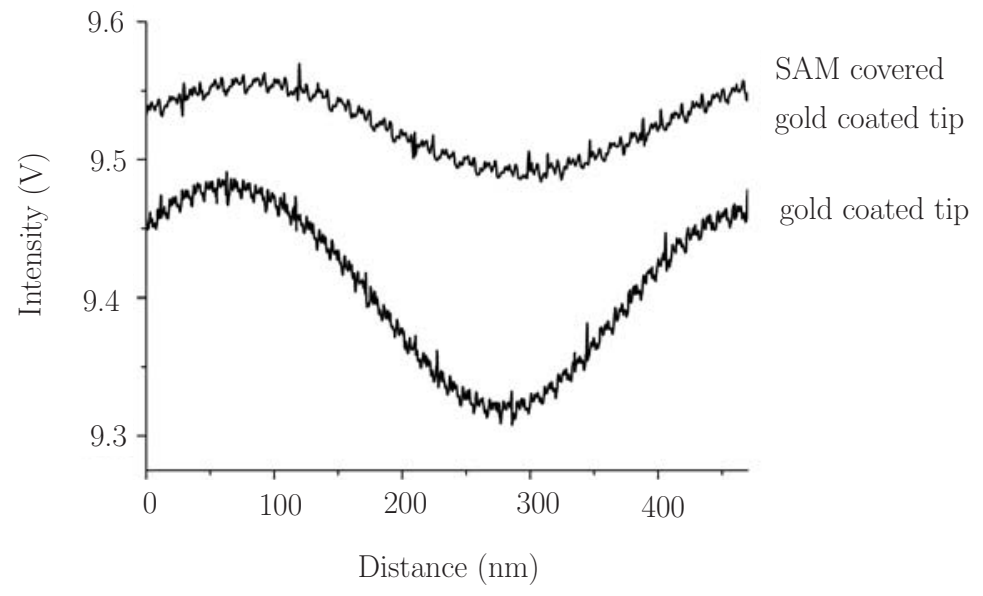

Figure 7.5: SNEM intensity-distance curves captured with bare gold coated and SAM of DoSH modified gold coated AFM tips. The optical signals for both AFM probe tips show oscillations with a $\lambda /(2 \cos \theta)$ periodicity. 


\subsection{Conclusions}

In this chapter, the effect of the long chain SAM coating on the SNEM contrast was further investigated. SAM of a DoSH covered gold coated AFM probe tip was used to image the microphase separated PS- $b$-PMMA diblock copolymer film. The tip was scanned at increasing pressures up to $0.82 \mathrm{GPa}$ to locally deform and displace the molecules at the tip edge where the tip is in contact with the sample.

The average amplitudes of the intensity variations calculated from the RMS deviations from the mean of four different areas showed increase upon increasing the force applied to scan the block copolymer film. As a result of the increasing imaging force, the SAM at the end of the tip was removed revealing the gold surface of the tip. Since the rest of the AFM probe was covered with SAM of DoSH, the unwanted reflections from the tip shaft was minimized. The decrease in the amplitude of the oscillations obtained by the intensity-distance curves for the SAM covered tip compared to gold coated tip has shown indication of the reduction of this far-field signal.

\subsection{Experimental section}

Materials:PS- $b$-PMMA diblock copolymer with a number average molar mass, $\mathrm{M}_{n}$, of 50000 and $47000 \mathrm{~g} \mathrm{~mol}^{-1}$ for the PS and PMMA block, respectively and a polydispersity index of 1.09 was purchased from Polymer Source Inc. (Montreal, Canada). DoSH (98\%) was obtained from Acros Organics (Geel, Belgium). Toluene and ethanol were purchased from Biosolve (Valkenswaard, The Netherlands). Hydrogen peroxide $\left(\mathrm{H}_{2} \mathrm{O}_{2}\right) 30 \%$ was obtained from Merck (Darmstadt, Germany). Sulfuric acid $\left(\mathrm{H}_{2} \mathrm{SO}_{4}\right)$ 95-97\% was bought from Sigma-Aldrich (St. Louis, MO, USA). All materials were used as received. Milli-Q water was produced by a Milipore Synergy system (Billereca, MA, USA).The glass slides were purchased from Thermo Scientific (Menzel-Glaser, Gerhard Menzel GmbH, Braunschweig, Germany). The glass slides were made of extra-white soda-lime glass. RMS roughness of a glass slides was $0.77 \mathrm{~nm}$ for a $1.5 \times 1.5 \mu \mathrm{m}^{2}$ area determined by AFM.

Sample preparation:The PS- $b$-PMMA diblock copolymer was dissolved in toluene (1 wt.\% solution) and spin coated on a glass slide at $2000 \mathrm{rpm}$ for 1 minute. Prior to spin coating the glass slide was cleaned with piranha solution (i.e. a solution of $1: 3(\mathrm{v} / \mathrm{v}) 30 \% \mathrm{H}_{2} \mathrm{O}_{2}$ and concentrated $\left.\mathrm{H}_{2} \mathrm{SO}_{4}\right)$ for 20 minutes and rinsed with copious amounts of high-purity water (Millipore Milli-Q water).Caution: Piranha solution is highly corrosive. Extreme care should be taken when handling Piranha solution and only small quantities should be prepared. The spin coated polymer film was annealed in air at $175{ }^{\circ} \mathrm{C}$ overnight [30, 31]. The film thickness was 35 $\mathrm{nm}$, as determined by AFM height measurements. 
Functionalization of the gold coated AFM tips: Silicon AFM probes (PointProbe Plus silicon probes, PPP-NCH, Nanosensors, Neuchatel, Switzerland) with average nominal resonance frequency and spring constant of $\sim 330 \mathrm{kHz}$ and $\sim 42 \mathrm{~N} \mathrm{~m}^{-1}$, respectively, were sputtered with a $13 \mathrm{~nm}$ thick layer of gold using a Balzers SCD 040 sputtering machine at an argon pressure of 0.05 mbar $(250 \mathrm{sec}$ sputtering at a $15 \mathrm{~mA}$ current). The cantilever spring constants were determined using the thermal tune method [32]. The average diameter of the SAM covered gold coated AFM probe tips was determined to be $24 \mathrm{~nm}$ by SEM images. A $10^{-3} \mathrm{M}$ solution of DoSH was prepared in ethanol. A freshly gold coated AFM probe was immersed in the DoSH solution for 16 hours at room temperature to allow the formation of a SAM on the gold surface. Subsequently, the probe was rinsed with ethanol and air-dried at room temperature.

SNEM:A MFP-3D AFM (Asylum Research, Santa Barbara, CA, USA) was combined with a Multiskop ellipsometer (Optrel, Sinzing, Germany) in the SNEM setup. Samples were placed on a custom-made interface plate (Optrel, Sinzing, Germany). Images were collected in air at AFM scan rates of $750 \mathrm{~nm} \mathrm{~s}^{-1}$ and $1024 \times 1024$ pixel $^{2}$ resolutions with tapping mode free amplitude of approximately $74 \mathrm{~nm}$ (peak to peak distance). The amplitude set point was maintained by a feedback loop at approximately $88 \%$ of the free oscillation amplitude. The resonance frequency of the SAM of DoSH modified gold coated AFM probe tip were $327 \mathrm{kHz}$. Measurements with increased imaging force and pressure were performed by gradually increasing the drive amplitude while maintaining the absolute amplitude set point value. The SNEM optical images were processed in Gwyddion by polynomial background leveling, followed by two-dimensional fast Fourier transform (2D-FFT) filtering, to remove unwanted periodic structures attributed to electronic noise at fixed frequencies [33].

SEM: The surface morphologies of the AFM probe tips were studied by field emission scanning electron microscopy (FE-SEM, JEOL, JSM-6330F) at $5 \mathrm{kV}$ of accelerating voltage. 


\section{Bibliography}

[1] Novotny, L.; Stranick, S. J. Annu. Rev. Phys. Chem. 2006, 57, 303-331.

[2] Keilmann, F. J. Electron Microsc. 2004, 53, 187-192.

[3] Rasmussen, A.; Deckert, V. Anal. Bioanal. Chem. 2005, 381, 165-172.

[4] Bailo, E.; Deckert, V. Chem. Soc. Rev. 2008, 37, 921-930.

[5] Deckert-Gaudig, T.; Deckert, V. Phys. Chem. Chem. Phys. 2010, 12, 1204012049.

[6] Asghari-Khiavi, M.; Wood, B. R.; Hojati-Talemi, P.; Downes, A.; McNaughton, D.; Mechler, A. J. Raman Spectrosc. 2012, 43, 173-180.

[7] Cui, X. D.; Zhang, W. H.; Yeo, B. S.; Zenobi, R.; Hafner, C.; Erni, D. Opt. Express 2007, 15, 8309-8316.

[8] Hillenbrand, R.; Keilmann, F. Phys. Rev. Lett. 2000, 85, 3029-3032.

[9] Hillenbrand, R.; Knoll, B.; Keilmann, F. J. Microsc-Oxford. 2001, 202, $77-$ 83.

[10] Hecht, B.; Bielefeldt, H.; Inouye, Y.; Pohl, D. W.; Novotny, L. J. Appl. Phys. 1997, 81, 2492-2498.

[11] Kaupp, G.; Herrmann, A.; Haak, M. J. Phys. Org. Chem. 1999, 12, 797-807.

[12] Weston, K. D.; Buratto, S. K. J. Phys. Chem. B 1997, 101, 5684-5691.

[13] Piner, R. D.; Zhu, J.; Xu, F.; Hong, S. H.; Mirkin, C. A. Science 1999, 283, 661-663.

[14] Ivanisevic, A.; Mirkin, C. A. J. Am. Chem. Soc. 2001, 123, 7887-7889.

[15] Ginger, D. S.; Zhang, H.; Mirkin, C. A. Angew. Chem. Int. Ed. 2004, 43, 30-45.

[16] Han, T.; Williams, J. M.; Beebe, T. P. Anal. Chim. Acta 1995, 307, 365-376.

[17] Schönherr, H.; Chechik, V.; Stirling, C. J. M.; Vancso, G. J. J. Am. Chem. Soc. 2000, 122, 3679-3687.

[18] Wold, D. J.; Frisbie, C. D. J. Am. Chem. Soc. 2001, 123, 5549-5556.

[19] Kiracofe, D.; Melcher, J.; Raman, A. Rev. Sci. Instrum. 2012, 83, 013702 $1-17$.

[20] Melcher, J.; Hu, S. Q.; Raman, A. Rev. Sci. Instrum. 2008, 79, 061301 1-11.

[21] Hertz, H. J. Reine Angev. Math. 1882, 9293, 156-171. 
[22] Schönherr, H.; Vancso, G. J. Scanning force microscopy of polymers; Springer: Berlin Heidelberg, 2010.

[23] Hay, J. L.; Wolff, P. J. J. Mater. Res. 2001, 16, 1280-1286.

[24] Salmeron, M. Tribol. Lett. 2001, 10, 69-79.

[25] Liu, G. Y.; Xu, S.; Qian, Y. L. Acc. Chem. Res. 2000, 33, 457-466.

[26] Barrena, E.; Ocal, C.; Salmeron, M. J. Chem. Phys. 2000, 113, 2413-2418.

[27] Carpick, R. W.; Salmeron, M. Chem. Rev. 1997, 97, 1163-1194.

[28] Salmeron, M.; Neubauer, G.; Folch, A.; Tomitori, M.; Ogletree, D. F.; Sautet, P. Langmuir 1993, 9, 3600-3611.

[29] David, T.; Chicanne, C.; Richard, N.; Krenn, J. R.; Scheurer, F.; Ounadjela, K.; Hehn, M.; Lacroute, Y.; Goudonnet, J. P. Rev. Sci. Instrum. 1999, 70, 4587-4594.

[30] Morkved, T. L.; Jaeger, H. M. Europhys. Lett. 1997, 40, 643-648.

[31] Zhang, X. H.; Berry, B. C.; Yager, K. G.; Kim, S.; Jones, R. L.; Satija, S.; Pickel, D. L.; Douglas, J. F.; Karim, A. Acs Nano 2008, 2, 2331-2341.

[32] Hutter, J. L.; Bechhoefer, J. Rev. Sci. Instrum. 1993, 64, 1868-1873.

[33] Necas, D.; Klapetek, P. Cent. Eur. J. Phys. 2012, 10, 181-188. 


\section{Chapter 8}

\section{Outlook for EC-IE and SNEM}

This chapter elaborates on possible future research directions for both hybrid methods, namely electrochemical imaging ellipsometry (EC-IE) and scanning near-field ellipsometric microscopy (SNEM). Firstly, the application of EC-IE for in situ monitoring of the morphology changes during an electrochemical synthesis is demonstrated by the example of polypyrrole (PPy) films. In this relation, the EC-IE is compared to the more often used in situ morphology characterization technique, namely the electrochemical atomic force microscopy (EC-AFM). Secondly, the potential of SNEM for sub-surface imaging is discussed. A preparation of a sample that consists of nanoparticles embedded below a flat polymer surface is explained. 


\subsection{Introduction}

IE is a fast non-destructive technique that not only allows one to quantify the thickness with high accuracy, but also visualize the thickness distributions and changes [1]. Electrochemical imaging ellipsometry (EC-IE), a hybrid method, allows real time in situ imaging of dynamic processes with sub-nanometer resolution in the vertical direction. In this thesis, the possibilities of this hybrid method are demonstrated by a study on the visualization of the morphology changes of a redox-responsive polymer, namely oligoethylene sulfide end-functionalized poly(ferrocenyldimethylsilane) (ES-PFS). A non-redox responsive MCU layer was used as an inert reference layer for the direct visualization of the morphology variation of the ES-PFS layer. A micro patterned sample of a ES-PFS/MCU was fabricated using micro-contact printing $(\mu \mathrm{CP})$. The micro patterned ES-PFS/MCU film was electrochemically oxidized/reduced and the response of the film was simultaneously monitored with IE. The thickness change was imaged in real time. The real time movie provided evidence of the two step thickness increase and reduction in accordance with the electrochemical mechanism of the redox reaction.

The EC-IE provides a vertical resolution in the sub-nanometer range. However, it is an objective based microscope and therefore, its lateral resolution is restricted by the diffraction limit of light. To achieve a high resolution in the lateral direction beyond the diffraction limit, one can use the light collection and field enhancement properties of a nanoscale metal probe. Using AFM for such a purpose is a beneficial approach since this long time existing well known method possess precise tip positioning. In the second hybrid technique, described in this thesis, AFM is combined with ellipsometry to achieve nanometer lateral resolution in optical imaging.

In SNEM, changes in the polarization state of the electromagnetic field, due to the interactions between a gold coated AFM tip and a sample, form the optical contrast [2]. The field enhancement is achieved due to plasmonic excitation and the lightening rod effect. The contrast formation of SNEM resembles IE since detection of the change in the polarization state of the reflected light from the sample surface forms the basis of both techniques.

In this chapter, possibilities of both EC-IE and SNEM instruments for future research are described in two sections. In the first section, a possible application of EC-IE for in situ morphology studies of electrochemical polymer film growth was explained. Advantages of EC-IE over the currently used technique to monitor the morphology change of polypyrrole $(\mathrm{PPy})$ during electrochemical synthesis were discussed. In the second section, the sub-surface imaging possibility of SNEM for the characterization of nanostructures that are embedded below a dielectric surface is discussed. A polyacrylic acid (PAA) polymer film, which contains amine functionalized silica nanoparticles below the sample surface, was prepared as a specific sample for sub-surface imaging. 


\subsection{Electrochemical imaging ellipsometry}

IE allows operation in liquid, change of the experiment environment such as temperature, $\mathrm{pH}$ and combination of other methods like electrochemistry [3, 4]. The EC-IE can give possibility not only to investigate electrochemical induced thickness change but also to monitor morphology changes during the electrochemical synthesis. For instance, the morphology changes of PPy films during electrochemical synthesis have been investigated using in situ electrochemical atomic force microscopy (EC-AFM) [5].

The morphology images showed that the growth of the PPy films occurred in two steps, i.e. deposition of a primary PPy layer followed by growth of this layer. Additionally, the EC-AFM data have revealed a highly orientated growth nuclei of PPy on a gold electrode. However, some difficulties which were associated with the measurement technique were mentioned. The pyrrole concentration was kept very low to slow down the synthesis so that the film growth could be observed with EC-AFM. Another study showed that the morphology of the film and the kinetics of the PPy synthesis can be affected by the oscillating AFM tip providing a horizontal scratching force and a vertical tapping force during the in situ ECAFM measurements [6].

It is believed that these challenges can be overcome using the EC-IE hybrid method. Since the EC-IE is a very fast scanning method (e.g., an area of 600 $\times 400 \mu \mathrm{m}^{2}$ can be imaged in 2 seconds), processes like film growth can be monitored without the necessity of slowing down the synthesis. Also, EC-IE is a noncontact method and therefore, the sample is not distorted during the measurement. Another advantage of EC-IE over the EC-AFM is the ability of EC-IE to determine the film thickness of the whole layer in addition to the morphology of the layer.

\subsection{Scanning near-field ellipsometric microscopy}

The ability of the apertureless scanning near-field optical microscopy (a-SNOM) to image nanoscale objects below the surface has not yet been fully explored. Taubner et al. have shown a study that provided an evidence towards subsurface imagining using a-SNOM [7]. In the study, an a-SNOM was used to image a sample which consists of a $25 \mathrm{~nm}$ high hexagonally arranged gold islands below a polystyrene (PS) layer.

It was found that the optical amplitude signal was low when scanning the PS areas, due to the low dielectric constant of the polymer. However, some areas revealed high optical amplitude but no topographical difference with the PS. These areas, also due to the similarities in shape, were associated with the gold islands below the PS layer. The thickness of the polymer layer that was above the gold islands, 
was reported as $5 \mathrm{~nm}$. However, the near-field optical depth was left unexplored in the study, expect stating that it is expected to be equal to the decay length of the near-field amplitude signal.

Based on its similarity in field enhancement, SNEM also possesses the potential of imaging nanoscale objects below the surface. Up to this date, subsurface imaging with SNEM has not yet been fully investigated except an optical signal recorded for silver nanoparticles within a PMMA polymer film in the absence of topographic contrast [8]. Imaging objects below the sample surface would open new possibilities in characterization of nano-assemblies, for instance nanoscale structures covered by a coating or functionalized by a polymer layer. In this section, attempts towards preparing a suitable sample to illustrate the sub-surface imaging using SNEM are provided.

The scheme of sub-surface imaging using an gold coated AFM probe tip is displayed in Fig. 8.1. The sample, shown in the scheme, is composed of nanoparticles which are embedded into a polymer film. Due to the limitation of the optical depth, the nanoparticles must be placed only a few nm below the surface. Therefore, a special sample preparation is required to illustrate the sub-surface imaging with SNEM.

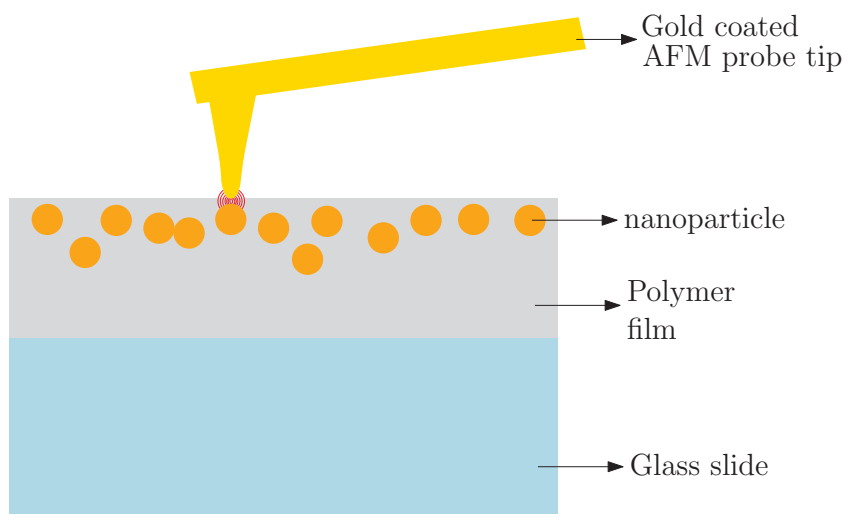

Figure 8.1: Schematic representation of subsurface imaging. Nanoparticles underneath the polymer film are illustrated. The red lines represent the near-field confined zone between the gold coated probe tip and the nanoparticles.

For the preparation of this sample, silica nanoparticles with modified surface chemistry were embedded into a polymer matrix. First, amine functionalized silica nanoparticles were dispersed on a poly(acrylic acid) (PAA) film by spin coating. These silica nanoparticles were well dispersed on the polymer film as displayed in the AFM topography image (Fig. 8.2(a)). The sample was then heated to elevated temperature above the $\mathrm{T}_{g}$ of the polymer film for a specific time, enough to sink the nanoparticles into the polymer surface (Fig. 8.2(b)). 
(a)

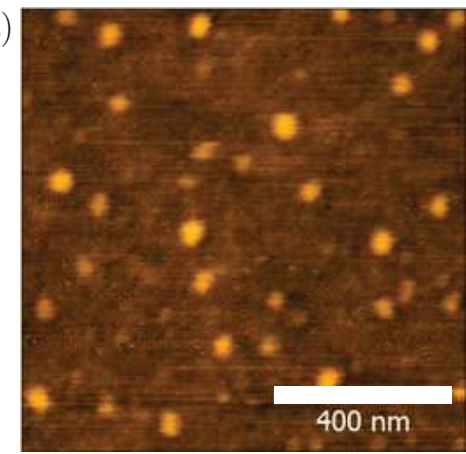

(b)

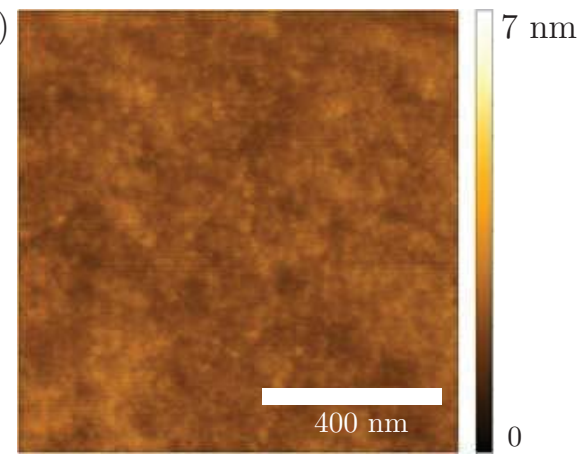

Figure 8.2: AFM height images of (a) before and (b) after heating at $145{ }^{\circ} \mathrm{C}$ for $19 \mathrm{~h}$. Amine functionalized silica nanoparticles dispersed on a PAA film.

The acid-base interactions between the amine groups on the silica nanoparticles and the acrylic acid groups of the polymer play an important role for nanoparticles to sink into the polymer [9]. Additionally, the embedding of the nanoparticles into a poly(methyl methacrylate-co-methacrylic acid) (PMMA-co-MAA) matrix with the molar proportions of methyl methacrylate to methacrylic acid 1:0.16 was investigated.

Fig. 8.3 shows the AFM height and phase images of the amine functionalized silica nanoparticles dispersed onto a PMMA-co-MAA matrix after heating the sample for 48 hours at $145{ }^{\circ} \mathrm{C}$. The AFM height image did not show large nanoparticles which were dispersed on the polymer matrix except a few particles that appear to be above the roughness of the polymer film. However, in the phase image (Fig. 8.3(b)), the nanoparticles were clearly distinguishable from the polymer matrix.

The height and the phase signals of two nanoparticles, indicated with numbers 1 and 2 in the images, were shown in the cross-sections (Fig. 8.3(c)). The nanoparticle indicated with number 1 , showed a height signal similar to the roughness of the polymer film with a slight depth on the sides and a tip in the middle. This indicates that the nanoparticle was partly sunk into the polymer film, but it was not completely buried. The nanoparticle, indicated with number 2, however, showed a higher AFM height signal than the roughness of the polymer film indicating that this nanoparticle is not completely below the polymer film surface neither.

Both nanoparticles showed significantly lower phase signal with the difference of nanoparticle number 2 revealing lower phase signal as it stuck out of the polymer surface more. Overall, the AFM height and phase images indicated that the amine functionalized silica nanoparticles were mainly located just beneath the surface but were not completely buried into the PMMA-co-MAA matrix. This was attributed to the low methacrylic acid content of the copolymer. 
(a)

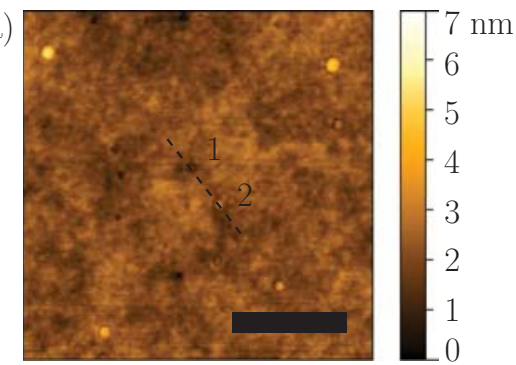

(b)

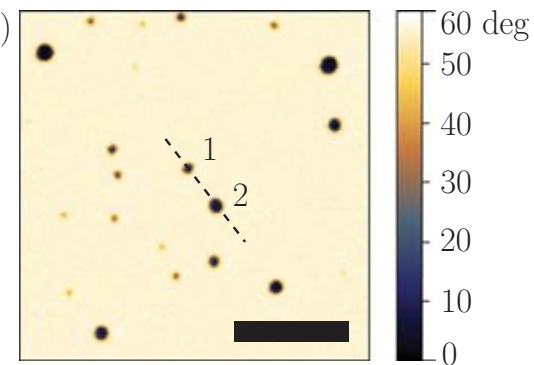

(c)

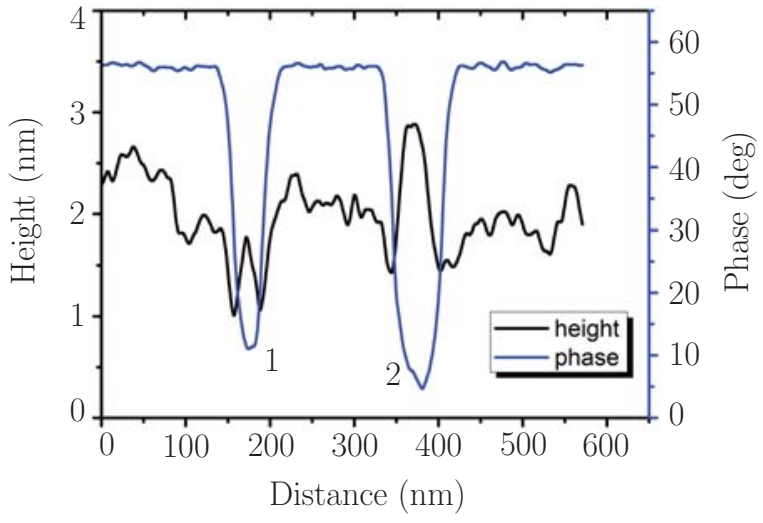

Figure 8.3: The AFM data of amine functionalized silica nanoparticles embedded into an PMMA-co-MAA film. The AFM height and AFM tapping mode phase images are shown in (a) and (b), respectively. In (c), the corresponding height (depicted in black) and phase (depicted in blue) cross-sections along the black dashed lines shown in the AFM images of (a) and (b) are displayed. Two silica nanoparticles are indicated with number 1 and 2 in the AFM images as well as in the cross-sections. The scale bar represents $500 \mathrm{~nm}$.

Note that the PAA $\left(\mathrm{M}_{w}\right.$ of $\left.15000 \mathrm{~g} / \mathrm{mol}\right)$ had a lower molar mass than the PMMAco-MAA polymer $\left(\mathrm{M}_{w}\right.$ of $\left.34000 \mathrm{~g} / \mathrm{mol}\right)$. Based on molar mass alone, the viscosity of the PAA at above its $\mathrm{T}_{g}$ would be lower than the PMMA-co-MAA polymer. However, it is known that polar interactions and hydrogen bonding occurs between carboxylic acid side groups which may increase melt viscosity [10, 11]. Viscosity will mainly influence the equilibrium time of the process of the nanoparticles sinking into the polymer matrix. Considering this effect, the nanoparticles, which were dispersed in PAA, were kept at above the $\mathrm{Tg}$ of the polymer shorter amount of time than those dispersed in the PMMA-co-MAA.

Embedding of 10 and $20 \mathrm{~nm}$ diameter bare gold spheres into polystyrene (PS) films were previously studied in the literature [12]. At temperatures above the $\mathrm{T}_{g}$ of the polymer, the surface dynamics of the polymer film is similar to those in the bulk of the liquid. It is stated in the literature that the spheres embed into the homogenous liquid film until the angle between the flat surface and the spheres is equal to the contact angle of the polymer and particle $[12,13]$. 
The surface tension forces are the leading driving force behind the embedding of the particles into the polymer film at $\mathrm{T}>\mathrm{T}_{g}$. The scheme in displayed in Fig. 8.4 illustrates the forces that act on the particle by the polymer meniscus. It is important to note that the forces due to gravity is much smaller than the surface tension forces and therefore they are neglected.

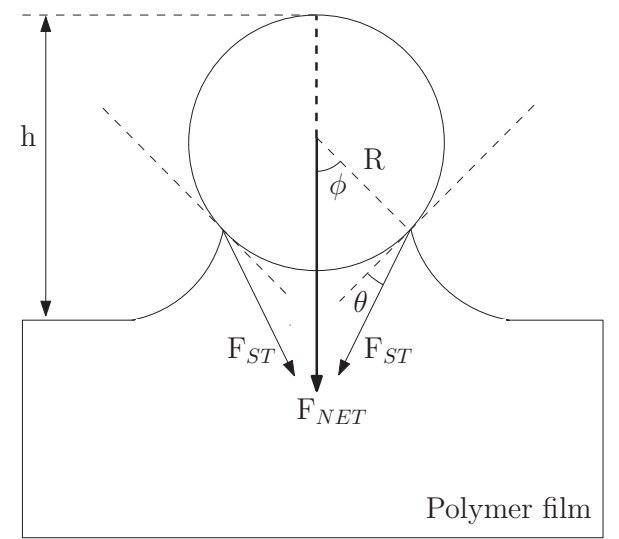

Figure 8.4: Scheme of the forces acting on a gold nanoparticle by a meniscus of a polymer film. The $\theta$ represent the equilibrium contact angle. The $\mathrm{F}_{N E T}$ represent the net downward forces acting on the particles.

Embedding of the bare nanoparticles stops at the angle between the flat surface of the polymer film and the sphere is equal to the particle/polymer contact angle [12]. However, in our case of the amine functionalized silica nanoparticles and the PAA film, the particles embed completely into the polymer film due to the interactions between the amine groups and the acrylic acid groups.

Due to the low difference between the refractive index of the silica nanoparticles $(n=1.47)[14]$ and the PAA matrix $(n=1.52)[15]$, the SNEM optical images of the buried silica nanoparticles did not display a significant optical contrast signal that can distinguish the silica nanoparticles below the PAA surface. The silica nanoparticles were chosen here to study sub-surface imaging of dielectric nanostructures within a dielectric matrix.

More research is needed to investigate the subsurface imaging possibilities of the SNEM. One should try the sample preparation described above with high refractive index nanoparticles for instance with $\mathrm{TiO}_{2}$ nanoparticles [16]. 


\subsection{Conclusions}

Preparation of a sample that contains nanoparticles embedded below a flat polymer film surface was described. Amine functionalized silica nanoparticles sank into a PAA film after heating the sample at $40{ }^{\circ} \mathrm{C}$ above its $\mathrm{T}_{g}$ for an efficient amount of time that is enough to complete the process. The same silica nanoparticles were also dispersed onto a PMMA-co-MAA film. AFM height and phase images of this sample showed that the particles were not completely buried under the polymer film. This was mainly attributed to the low methacrylic acid content of PMMA-co-MAA.

The buried silica nanoparticles did not show a distinguishable optical contrast against the PAA film in SNEM images. This was attributed to the low refractive index difference of the silica nanoparticles and the polymer film. More study must be carried out to investigate the subsurface imaging ability of SNEM. Our suggestion is to use $\mathrm{Au}$ or $\mathrm{TiO}_{2}$ nanoparticles since the these structures are highly polarizable and should provide a stronger optical signal within a low polarizable matrix.

\subsection{Experimental section}

Materials: Poly(acrylic acid, sodium salt) solution (35 wt.\% in water, $\mathrm{M}_{w}$ of 15000 $\mathrm{g} / \mathrm{mol}$ ) was purchased from Sigma Aldrich. It was turned into its carboxylic acid form by treatment with dilute $\mathrm{HCl}$, using dialysis. PMMA-co-MAA polymer $\left(\mathrm{M}_{w}\right.$ of $34000 \mathrm{~g} / \mathrm{mol}$ ) was also obtained from Sigma Aldrich and was used as received. Tetraethylorthosilicate (TEOS, 98\%), (3-Aminopropyl)triethoxysilane (APTES, $99 \%$ ) and ammonium hydroxide $\left(\mathrm{NH}_{4} \mathrm{OH}, 28-30 \% \mathrm{NH}_{3}\right.$ basis) were provided from Sigma Aldrich and used as received.

Sample preparation: Colloidal silica nanoparticles were prepared by using $0.8 \mathrm{ml}$ TEOS and $0.8 \mathrm{ml} \mathrm{NH}_{4} \mathrm{OH}$ in $25 \mathrm{ml}$ ethanol solution. The mixture was stirred at room temperature for $24 \mathrm{~h}$. The particle size was determined by dynamic light scattering (DLS, Zetasizer 4000, Malvern Instruments, Malvern, UK) at $25{ }^{\circ} \mathrm{C}$ found to be around $30 \mathrm{~nm}$. After centrifuged the particles were amine functionalized by reacting APTES $(1 \mathrm{ml})$ with the native hydroxyl groups on the colloidal silica nanoparticles. The particle size of the amine functionalized silica nanoparticles were found to be around $80 \mathrm{~nm}$ by DLS.

PAA was dissolved in water and spin coated on a glass slide at $2000 \mathrm{rpm}$. The polymer film was dried overnight under vacuum at $80^{\circ} \mathrm{C}$ to remove water residue. Amine functionalized silica nanoparticles were dispersed in toluene at 0.1 wt.\% and sonicated for 45 minutes. A drop of the dispersion was placed on the polymer film right after sonication. A freshly piranha cleaned silicon wafer was placed on the droplet to spread the dispersion on the polymer film. This sandwich was 
placed into the vacuum oven overnight to evaporate the solvent. The silicon wafer was removed and the nanoparticles were imaged by AFM. The sample was then placed into an oven at $145{ }^{\circ} \mathrm{C}$ for 19 hours for the embedding of the nanoparticles. The same sample preparation procedure was for PMMA-co-MAA (10 wt.\% solution in dioxane) and $0.1 \mathrm{wt} . \%$ amine functionalized silica particles in toluene solution expect that this sample was placed in the oven $48 \mathrm{~h}$ at $145^{\circ} \mathrm{C}$.

AFM: AFM measurements were performed using a Dimension D3100 AFM operated with a NanoScope IVa controller (Digital Instruments/Bruker, Santa Barbara, CA, USA). Tapping mode AFM images were collected with silicon cantilevers/tips (PointProbe Plus silicon probes, PPP-NCH, Nanosensors, Neuchatel, Switzerland) in air. The system operating frequency was typically $10 \%$ lower than the natural resonance frequency of the cantilever in air, the free amplitude was kept constant, while the set point amplitude was approximately $86 \%$ of the free amplitude $(\sim 2.0 \mathrm{~V})$. 


\section{Bibliography}

[1] Asinovski, L.; Beaglehole, D.; Clarkson, M. T. Phys. Status Solidi A 2008, 205, 764-771.

[2] Cumurcu, A.; Duvigneau, J.; Lindsay, I. D.; Schön, P. M.; Vancso, G. J. Eur. Polym. J. 2013, 49, 1935-1942.

[3] Schmaljohann, D.; Nitschke, M.; Schulze, R.; Eing, A.; Werner, C.; Eichhorn, Y. J. Langmuir 2005, 21, 2317-2322.

[4] Svoboda, V.; Cooney, M. J.; Rippolz, C.; Liaw, B. Y. J. Electrochem. Soc. 2007, 154, D113-D116.

[5] Suarez, M. F.; Compton, R. G. J. Electroanal. Chem. 1999, 462, 211-221.

[6] Cai, X. W.; Gao, J. S.; Xie, Z. X.; Xie, Y.; Tian, Z. Q.; Mao, B. W. Langmuir 1998, 14, 2508-2514.

[7] Taubner, T.; Keilmann, F.; Hillenbrand, R. Opt. Express 2005, 13, 88938899.

[8] Tranchida, D.; Diaz, J.; Schön, P.; Schönherr, H.; Vancso, G. J. Nanoscale 2011, 3, 233-239.

[9] Qu, M.; Meth, J. S.; Blackman, G. S.; Cohen, G. M.; Sharp, K. G.; Van Vliet, K. J. Soft Matter 2011, 7, 8401-8408.

[10] Otocka, E. P.; Kwei, T. K. Macromolecules 1986, 1, 244-249.

[11] Kang, N.; Xu, Y. Z.; Wu, J. G.; Feng, W.; Weng, S. F.; Xu, D. F. Phys. Chem. Chem. Phys. 2000, 2, 3627-3630.

[12] Sharp, J. S.; Teichroeb, J. H.; Forrest, J. A. Eur. Phys. J. E 2004, 15, 473-487.

[13] Teichroeb, J. H.; Forrest, J. A. Phys. Rev. Lett. 2003, 91, 016104 1-4.

[14] Khlebtsov, B. N.; Khanadeev, V. A.; Khlebtsov, N. G. Langmuir 2008, 24, 8964-8970.

[15] Gooch, J. W. Encyclopedic dictionary of polymers; Springer: New York, 2007.

[16] Auvinen, S.; Alatalo, M.; Haario, H.; Vartiainen, E.; Jalava, J. P.; Lamminmaki, R. J. J. Phys. Chem. C 2013, 117, 3503-3512. 


\section{Summary}

In this thesis, hybrid methods for nanoscale characterization of heterogeneous thin polymer films were discussed. Essentially two ellipsometry based hybrid methods were established or further developed, respectively, namely electrochemical imaging ellipsometry (EC-IE) and scanning near field ellipsometry microscopy (SNEM). Ellipsometry was combined with electrochemical methods for the visualization of redox driven dynamic changes in thin polymer films. In particular, the redox responsive behaviour of an oligoethylene sulfide end-functionalized poly(ferrocenyldimethylsilane) (ES-PFS) film was investigated with this hybrid method against a passive (non-redoxactive) 11-mercapto-1-undecanol (MCU) layer. The thickness change of the PFS layer upon oxidation and the reduction was observed in situ in real time with EC-IE.

This hybrid method provides vertical resolution at the sub nanometer scale while its lateral resolution is limited to the wavelength of the light source $(\lambda=633 \mathrm{~nm})$. In contrast, SNEM reveals lateral resolution below the diffraction limit of the light. The emphasis of the thesis was devoted to SNEM, including the introduction of the instrument, experimental case studies to enlighten its contrast formation and the optimization of the method. SNEM enables optical contrast imaging of thin transparent polymer films at the nanoscale due to the combination of the spatial tip-sample positioning capability of the atomic force microscopy (AFM) together with the high sensitivity of ellipsometry.

The motivation and the introduction of the topics that are covered in this thesis were presented in Chapter 1. The need for hybrid methods for the characterization of the nanoscale structures and the role of the hybrid methods used in this thesis were also provided in this chapter. To deliver background information on the hybrid techniques used in this thesis, Chapter 2 was dedicated not only to the summary of recent developments but also to the fundamentals of the imaging ellipsometry (IE) and various scanning near-field optical microscopy (SNOM) techniques. Firstly, the basics of the far-field and near-field microscopy as well as the diffraction limit of light were explained, but the emphasis was put on the IE and its applications in polymer characterization. Lastly, the apertureless and apertured SNOM techniques were described focusing mainly on tip enhanced techniques such as tip-enhanced Raman scattering (TERS) and SNEM. 
In Chapter 3, the first hybrid setup used in this thesis, the EC-IE, was introduced. This method combines IE with electrochemical methods for real time imaging of electrochemical responds of thin films. In this chapter, a study on monitoring in-situ thickness changes of ES-PFS relative to a molecular reference layer, the MCU layer, was provided. The ES-PFS/MCU micro-patterned sample was fabricated using micro-contact printing $(\mu \mathrm{CP})$. Ellipsometric contrast images of the micro-patterned ES-PFS layer/MCU film were recorded in situ with a closed electrochemical liquid cell.

The second hybrid method, the SNEM, was explained in Chapter 4 including its experimental arrangement, the optical contrast mechanism and the field enhancement. Also, the contrast formation based on a model, termed point dipole model, which describes the interactions between the tip and the sample for apertureless SNOM techniques was explained. The model calculations were compared to the experimental results in this thesis. Lastly this chapter covered the background scattering which originates from the multiple reflections between the large illuminated areas of the cantilever and the sample surface.

SNEM images of a microphase separated nanoscale morphology of a poly(styrene$b$-2-vinyl pyridine) (PS- $b$-P2VP) diblock copolymer film was studied in Chapter 5 to better understand the SNEM optical contrast mechanism. For instance, the importance of using a gold coated probe tip for the field enhancement was shown by comparing SNEM images obtained with a gold coated and a bare silicon probe tip. The dielectric constant variation driven nature of the SNEM optical contrast images was shown by comparing the SNEM images of a PS- $b$-P2VP copolymer film which were captured before and after iodine staining. By staining this block copolymer with iodine, the $\mathrm{P} 2 \mathrm{VP}$ domain reacts with iodine resulting in a domain with higher dielectric constant. The PS domain, however, did not change its configuration and therefore, also the dielectric constant upon iodine staining. Lastly, the effect of the tip sample distance to the optical contrast was investigated by recording SNEM optical images at various tip sample distances. The experimental results in this chapter were compared to the point dipole model calculations.

The lifetime of the gold coated probe tips, used in SNEM measurements, was investigated in Chapter 6. The gold coated AFM probe tips lose their activity over time when stored in ambient air. Therefore, SNEM optical data obtained with a freshly prepared metal coated probe and an aged probe are not directly comparable. To prolong the lifetime of the metal coated probes, a self-assembled monolayer (SAM) of ethanethiol (EtSH) was used as a protective coating. Performance of the gold coated and the SAM protected gold coated probe tips were measured by comparing the amplitude of the intensity variations of the SNEM images collected with these probe tips. A microphase separated polystyreneblock-poly(methylmethacrylate) (PS- $b$-PMMA) diblock copolymer film was used as a sample in this chapter. On one hand, the SNEM images of the PS- $b$-PMMA diblock copolymer film, which were recorded with a gold coated AFM probe tip, showed $66 \%$ decrease in the amplitude of the intensity variations after storing the 
AFM probe tip five days in ambient air. On the other hand, the optical images, which were recorded with a SAM of EtSH protected AFM probe tip, showed only $14 \%$ decrease in the amplitude of the intensity variations over the same time and storage conditions. Additionally, alkanethiols of various lengths were used to modify gold coated AFM probe tips to determine the thiol length that diminished the intensity variations in the optical image. By increasing the length of the alkanethiol of the protective layer, the amplitudes of the intensity variations of the SNEM images captured with the respective modified AFM probe tip decreased.

In Chapter 7, the role of the effects of the SAM of 1-dodecanethiol (DoSH) coating on SNEM contrast was further investigated. Modifying the gold coated probe tip with a long chain thiol SAM of DoSH blocked the optical contrast due to the dielectric shielding of the gold coated probe tip via the densely packed SAM. The SAM molecules at the tip apex were then removed by scanning at increasing pressures. The amplitude of the intensity variations was increased in the SNEM images revealing the block copolymer domain nanostructures by removing the SAM layer. The decrease of the background scattering was seen by the intensitydistance curves. Additionally, the outlook with a discussion of the possibilities of both methods for future reseach was provided in Chapter 8. 


\section{Samenvatting}

In deze thesis, hybride methodes voor de karakterisering van heterogene dunne polymeerlagen op nanoschaal zijn beschouwd. Twee hybride methodes gebaseerd op ellipsometrie zijn gerealiseerd of verder ontwikkeld, respectievelijk "electrochemical imaging ellipsometry (EC-IE)" en "scanning near field ellipsometry microscopy (SNEM)".

Ellipsometrie is gecombineerd met elektrochemische methodes voor de visualisering van redox gedreven dynamische veranderingen in dunne polymeerlagen. Het redox gedreven responsief gedrag van een oligoethylene sulfide end-functionalized poly(ferrocenyldimethylsilane) (ES-PFS) film is onderzocht met deze methode en vergeleken met een passieve, niet redox actieve, 11-mercapto-1-undecanol (MCU) laag. De dikteverandering van de PFS laag gedurende oxidatie en reductie is geobserveerd in situ en in real-time met EC-IE.

Deze hybride methode verschaft een verticale resolutie op nanometer schaal terwijl de laterale resolutie gelimiteerd is door de golflengte van de lichtbron $(\lambda=$ $633 \mathrm{~nm}$ ). In tegenstelling tot EC-ICE, geeft SNEM een laterale resolutie lager dan de diffractielimiet van het licht. Het belangrijkste aandachtsgebied van deze thesis is de SNEM methode, inclusief de introductie van het instrument, experimentele case studies ter verheldering van de contrastvorming en optimalisering van de methode. SNEM heeft de mogelijkheid om contrastbeeldvorming op nanoschaal uit te voeren op dunne transparante polymeerlagen door de ruimtelijke tip-monster positioneringsmogelijkheid van een atoomkrachtmicroscoop (AFM) te combineren met de hoge gevoeligheid van ellipsometrie.

De motivatie voor dit onderzoek en de inleiding van de onderwerpen die in deze thesis aan bod komen worden gepresenteerd in Hoofdstuk 1. The noodzaak voor hybride methodes voor de karakterisering van structuren op nanometer schaal en de rol van de hybride methodes die in deze thesis gebruikt werden, werden beschreven in dit hoofdstuk. Om achtergrondinformatie te verschaffen over de gebruikte hybride methodes in deze thesis is Hoofdstuk 2 niet alleen toegewijd aan de samenvatting van recente ontwikkelingen maar ook aan de grondbeginselen van imaging ellipsometrie (IE) en verschillende scanning near-field microscopie (SNOM) technieken. Eerst werd de basis van de far-field en near-field microscopie 
alsook de diffractielimiet van het licht uitgelegd, maar de belangrijkste aandacht werd gegeven aan de IE en de toepassing in polymeerkarakterisering. Daarna werden de SNOM technieken met en zonder diafragma beschreven, waarbij de aandacht vooral gericht werd op sonde verbeterde technieken zoals "tip-enhanced Raman scattering" (TERS) en SNEM.

In Hoofdstuk 3, de eerste hybride opstelling die gebruikt werd in de thesis is gentroduceerd, namelijk de EC-IE. Deze methode combineert IE met elektrochemische methodes voor de real-time beeldvorming van elektrochemische reacties van dunne films. In dit hoofdstuk werd een in-situ studie naar dikteveranderingen van ES-PFS relatief aan een moleculaire referentie laag, MCU, beschreven. Het ES$\mathrm{PFS} / \mathrm{MCU}$ micro-patroon iss gefabriceerd door middel van micro-contact printen $(\mu \mathrm{CP})$. Ellipsometrie contrast beelden van de micro-patroon ES-PFS/MCU laag zijn opgenomen in-situ met een gesloten elektrochemische cel voor vloeistoffen.

De tweede hybride methode, de SNEM, is uitgelegd in Hoofdstuk 4, inclusief de experimentele inrichting, het optische contrast mechanisme en de verbetering van het electromagnetische veld. De contrastvorming werd ook verklaard door middel van een model dat de interacties tussen de sonde en het oppervlakte beschrijft voor diafragmaloze SNOM technieken. Deze modelberekeningen zijn vergeleken met de experimentele resultaten in deze thesis. Verder is in dit hoofdstuk ook de achtergrondsverstrooiing beschreven die ontstaat ten gevolge van verschillende reflecties tussen de grote verlichte gebieden van de cantilever en het monsteroppervlak.

In Hoofdstuk 5, zijn de SNEM beelden van een door microfase gescheiden morfologie van een poly(styrene- $b$-2-vinyl pyridine) (PS- $b$-P2VP) diblok copolymeerfilm bestudeerd voor een beter begrip van het SNEM optisch contrast mechanisme. Het belang van het gebruik van een goud gecoate sondetip voor veld verbetering, bijvoorbeeld, is aangetoond door het vergelijken van SNEM beelden verkregen met een goud gecoate sonde en een kale silicium sonde. De variatie in dilektrische constante van de SNEM optische contrast beelden zijn aangetoond door het vergelijken van SNEM beelden van een PS- $b$-P2VP copolymeerfilm voor en na jodiumkleuring. Door de kleuring van dit blok copolymeer met jodium reageert het P2VP domein met het jodium wat resulteert in een domein met een hogere dilektrische constante. Het PS domein, echter, verandert zijn configuratie niet en daarom ook niet zijn dilektrische constante. Tenslotte is het effect van de sonde-monster afstand op het optische contrast onderzocht door middel van het opnemen van SNEM optische contrast beelden bij verschillende tip-monster afstanden. De experimentele resultaten in dit hoofdstuk zijn vergeleken met de punt-dipool modelberekeningen.

De levensduur van de goud gecoate sondetips gebruikt in SNEM metingen werd onder de aandacht gebracht in Hoofdstuk 6. De goud gecoate sondetips verloren hun activiteit na enige tijd wanneer ze bewaard werden in een atmosferische omgeving. Daarom zijn SNEM optische data verkregen met een vers voorbereide metaal gecoate sondetip en een verouderde sondetip niet direct vergelijkbaar. 
Om de levensduur van de metaal gecoate sondetips te verlengen werd een zelf geassembleerde monolaag (SAM) van ethaanthiol (EtSH) gebruikt als beschermende coating. De prestaties van de goud gecoate en met SAM beschermende goud gecoate sondetips zijn gemeten door de amplitude van de intensiteitsvariaties van de SNEM beelden verkregen met deze sondetips te vergelijken. Een microfase gescheiden polysterene-block-poly(methylmethacrylate) (PS- $b$-PMMA) diblok copolymeer film werd gebruikt als monster in dit hoofdstuk. De SNEM beelden van de PS- $b$-PMMA diblok copolymeerfilm, die opgenomen waren met de goud gecoate AFM sondetip die vijf dagen in omgevingslucht was opgeslagen, gaven een $66 \%$ verlaging in de amplitude van de intensiteitsvariaties. De optische beelden die opgenomen waren met een beschermde AFM sondetip met een SAM van EtSH gaven enkel een 14\% verlaging in de amplitude van de intensiteitsvariaties na gelijke opslagcondities. Bovendien werden alkaanthiols van verschillende lengtes gebruikt om de goud gecoate AFM sondetips te modificeren om te achterhalen welke thiol lengte de intensiteitsvariaties in de optische beelden vermindert. Door het verhogen van de lengte van het alkaanthiol van de beschermende laag werden de amplitudes van de intensiteitsvariaties van de SNEM beelden opgenomen met de gemodificeerde AFM sondetip verminderd.

De effecten van een SAM van 1-dodecanethiol (DoSH) op het SNEM contrast is verder onderzocht in Hoofdstuk 7. Modificering van de goud gecoate sondetip met een lange thiol SAM van DoSH blokkeerde het optische contrast ten gevolge van de dilektrische afscherming van de goud gecoate sondetip door de dicht op elkaar gepakte SAM. De SAM moleculen op de sondetip top waren verwijderd door het scannen met verhoogde scandruk. Door het verwijderen van de SAM laag waren de amplitudes van de intensiteitsvariaties verhoogd in de SNEM beelden, hetgeen de blok copolymeer domeinstructuren onthulde. De vermindering van de achtergrondsverstrooiing werd aangetoond door de intensiteit-afstand krommes. De vooruitzichten met discussie over de mogelijkheden van beide hybride methodes voor verder onderzoek zijn beschreven in Hoofdstuk 8. 


\section{Acknowledgements}

The work that has been described in this thesis would not be possible without the help of many people. Therefore, I want to take this opportunity to thank all of them.

I first would like to thank Prof. Vancso for giving me the chance to work in his group as a PhD student. Your work attitude and enthusiasm inspired me. Your approach of setting high standards made me push my boundaries to achieve better. These values I have gained during my $\mathrm{PhD}$ not only helped me to achieve this thesis, but I believe they will also stay with me to help accomplish new successes. You also motivated me to build collaborations, which all ended up with success and improved this thesis greatly. Additionally, you supported me in deciding my future career direction. Your feedback and interest made my job-hunting period much more easier.

I wish to thank my daily supervisor Peter, for his help and encouragement. You have been understanding and supportive in all stages of my PhD. I also want to specially thank for your tips on how to prepare for the job interviews. They were very useful.

During my PhD, I had great collaborations and people who supported me. Firstly, I gratefully appreciate the efforts of Dr. Ian Lindsay and his support during the time I spend in Bristol as a visiting researcher. Secondly, I thank Sissi, Jordi, Joost, Mark, and Davide for their help, suggestions and positive comments. Lastly, I am grateful for the comments of Prof. Holger Schönherr and Prof. Pepijn Pinkse. I also thank Mine for her support in the first year of my PhD. It was a pleasure working with you.

I thank all my colleagues in the MTP group. I want to start with thanking my paranymph Xueling. In addition to the scientific discussions, you were a good supporting friend to talk to. I wish you the best of success with your thesis. I believe that you will achieve your future plans. Furthermore, I thank my second paranymph and friend Lionel. Thanks for your support to the PFS project. Also, for the fun conversations during the coffee breaks and letting me crash at your place the time when I locked myself out of my apartment. The nights out in 
Enschede, BBQ's and keeping up with my tennis talent(!) are things that I will not soon forget. I hope to still keep in touch.

I had a pleasure of working with many nice people. Starting with who are no longer in the MTP group, Edit, thanks for showing me how to prepare SAM, getting your expertise on this made my job much easier. You have been a fun person to talk to and we will see each other more in Eindhoven from now on. Wilma, thanks for the coffee break conversations and asking me to be your paranymph. It was nice to be a part of the ceremony. Eddy, thanks for showing up out of nowhere in the lab and making the time in Enschede more fun.

Continuing with my colleagues who are still in the MTP group, my officemate, Bart, thanks for translating the summary of the thesis to Dutch and putting a sign (keep it close) on the door of our office, so I can quietly write my thesis. Sissi, it was great to have you as an officemate around the end of my $\mathrm{PhD}$, your positive attitude made a difference in the atmosphere of the office. Also Michel, Hairong, Kaihuan, Bram, Andreas, Shanqiu and Yunlong thanks for being great colleagues. I thank Clemens for his excellent support on many things ranging from fixing every problem of the setup in no time to repairing the flat tire of my bike. Additionally, Genevieve and Hanke, thank you both for the help with all the arrangements.

Tezimin son kısmındaki teşekkür notunu sevgili aileme ayırmak istiyorum. Değerli anne ve babacı̆̆ım, diğer başarılarımda olduğu gibi doktoramıda sizlerin büyük emeklerine borçluyum. Özellikle doktora yapma konusunda beni yönlendirdiğiniz ve her aşamasında bana güvenip desteklediğiniz için sizlere teşekkür ederim. Doktoramın son dönemlerinde sizlerin her skype konuşmamızda o dün yaptiğin deneylerin sonuçları nasil çıktı diye sorup adım adım deneylerimi takip etmeniz bana büyük destek verdi. Canım kardeşim Gülsah, dünyada kardeşden daha tatlı birşey yoktur ve senden de daha tatl bir kardeş yoktur. Ayrıca abilerim Mustafa ve Suat'a, Hülya ablama ve de biricik halama bana olan destek ve hiç eksik etmedikleri sevgilerinden dolayı teşekkür ederim.

Ook wil ik Gerda en Nico bedanken voor al hun steun en zorg, het inrichten van mijn appartement en daarna ook weer het leeghalen. Ook voor al hun hulp aan het bouwen van mijn nieuwe thuis, ze geven me het gevoel dat ik ook een familie in België heb.

Lastly, dear Bart, see I am finally finishing my PhD. You have not only gave me encouragement and strenght, but also helped me every step of the way (finding the position till writing the thesis). I believe this end is a new begining and having you by my side makes me more excited for the future. Thanks for everything.

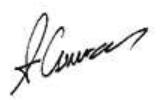




\section{Curriculum Vitae}

Aysegul Cumurcu was born on the $12^{\text {th }}$ of October, 1981 in Istanbul, Turkey. She obtained her bachelor degree in Chemical Engineering from Istanbul University in 2005. Later, she spent a year in Vancouver, Canada attending language courses at the Canadian College and Pacific Language Institute.

In 2006, she returned to Istanbul and started her master's degree in Chemical Engineering at Istanbul Technical University (ITU). In 2008, she continued her research project

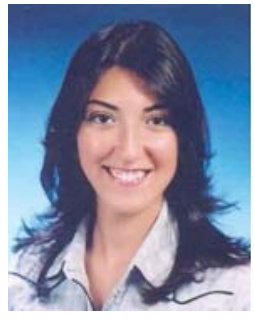
at the Eindhoven University of Technology (TU/e), Eindhoven, The Netherlands in the group of Prof. Dr. C.E. (Cor) Koning as a master's degree research intern. She worked on synthesis and characterization of polystyrene-nylon block copolymers and this project was issued in the PhD thesis of Dr. Simona Jarmina Ilinca.

Aysegul has completed her master's degree with a thesis entitled: 'Synthesis and properties of alkoxysilane-functionalized urethane oil/titania hybrid films' under the supervision of Prof. Dr. Tuncer Erciyes in 2009.

Starting from March 2010, Aysegul has been working as a PhD candidate at the University of Twente in Enschede, The Netherlands, in the Material Science and Technology of Polymers (MTP) group headed by Prof. Dr. G. Julius Vancso. Her research project was funded by the Dutch Polymer Institute (DPI).

In 2011, she stayed at the University of Bristol, Bristol, UK in the group of Nanophysics and Soft Matter as a visiting researcher. During this time she worked with Dr. Ian D. Lindsay on applying point dipole model simulations to polymer materials to define tip-sample interactions of tip-enhanced near-field optical microscopy. Results of this collaboration was also described in this thesis.

Aysegul is currently working as an Application \& Business Support Engineer at ASML in Veldhoven, The Netherlands. 


\section{List of publications}

1. Cumurcu, A.; Diaz, J.; Lindsay, I. D.; De Beer, S.; Duvigneau, J.; Schön, P. M.; Vancso, G. J., Optical imaging beyond the diffraction limit by SNEM: Effects of AFM tip modifications with thiol monolayers on imaging quality, submitted to Ultramicroscopy.

2. Cumurcu, A.; Feng, X. L.; Dos Ramos, L.; Hempenius, M. A.; Schön, P. M.; Vancso, G. J., Sub-nanometer expansions of redox responsive polymer films monitored by imaging ellipsometry. Nanoscale 2014,

DOI: $10.1039 / \mathrm{C} 4 \mathrm{NR} 02852 \mathrm{~J}$.

3. Cumurcu, A.; Duvigneau, J.; Lindsay, I. D.; Schön, P. M.; Vancso, G. J., Multimodal imaging of heterogeneous polymers at the nanoscale by AFM and scanning near-field ellipsometric microscopy. Eur Polym J 2013, 49 (8), $1935-1942$

4. Cumurcu, A.; Duvigneau, J.; Lindsay, I. D.; Schön, P. M.; Vancso, G. J., Nanoscale scanning near-field ellipsometric microscopy (SNEM) imaging of heterogeneous polymers. Proceeding MRS fall meeting 2013, 1562 mrsf131652-l106-03 doi:10.1557/opl.2014.261.

5. Feng, X. L.; Cumurcu, A.; Sui, X. F.; Song, J.; Hempenius, M. A.; Vancso, G. J., Covalent layer-by-layer assembly of redox-active polymer multilayers. Langmuir 2013, 29 (24), 7257-7265.

6. Cumurcu, A.; Yazgan, P.; Erciyes, A. T., Synthesis and properties of alkoxy silane-functionalized styrenated oil based polyester. Prog Org Coat 2011, 71 (2), 147-152.

7. Cumurcu, A.; Erciyes, A. T., Synthesis and properties of alkoxysilanefunctionalized urethane oil/titania hybrid films. Prog Org Coat 2010, 67 (3), 317-323. 